\title{
Sutured Floer homology, sutured TQFT and noncommutative QFT
}

\author{
DANIEL V MATHEWS
}

\begin{abstract}
We define a "sutured topological quantum field theory", motivated by the study of sutured Floer homology of product 3-manifolds, and contact elements. We study a rich algebraic structure of suture elements in sutured TQFT, showing that it corresponds to contact elements in sutured Floer homology. We use this approach to make computations of contact elements in sutured Floer homology over $\mathbb{Z}$ of sutured manifolds ( $D^{2} \times S^{1}, F \times S^{1}$ ) where $F$ is finite. This generalises previous results of the author over $\mathbb{Z}_{2}$ coefficients. Our approach elaborates upon the quantum field theoretic aspects of sutured Floer homology, building a noncommutative Fock space, together with a bilinear form deriving from a certain combinatorial partial order; we show that the sutured TQFT of discs is isomorphic to this Fock space.
\end{abstract}

57M50; 57R58, 57M27, 57R56

\section{Introduction}

\subsection{Chord diagrams and signs}

This paper, like its prequel [17], is about fun with chord diagrams. A chord diagram $\Gamma$ is a finite collection of nonintersecting properly embedded arcs in a 2-dimensional disc $D^{2}$, up to homotopy relative to endpoints. Fixing $2 n$ points on $\partial D^{2}$, the number of chord diagrams of $n$ arcs (or chords) connecting them is $C_{n}$, the $n$-th Catalan number. In [17] we considered the $\mathbb{Z}_{2}$ vector space $\operatorname{SFH}_{\text {comb }}(T, n)$ generated by chord diagrams of $n$ chords, subject to a relation called the bypass relation. The bypass relation says that whenever 3 chord diagrams coincide, except within a disc on which the chords appear as in Figure 1, they sum to zero.

In [17], we showed that this vector space describes precisely contact elements in the sutured Floer homology (SFH) of $\left(D^{2} \times S^{1}, F \times S^{1}\right)$, where $|F|=2 n$, with $\mathbb{Z}_{2}$ coefficients. However SFH can be defined over $\mathbb{Z}$; our vector space $S F H_{\text {comb }}$ should generalise to $\mathbb{Z}$ coefficients.

Over $\mathbb{Z}$, contact elements have a sign ambiguity; see Honda, Kazez and Matić [10]. So a chord diagram should represent an element in an abelian group $V$, up to sign, as in 

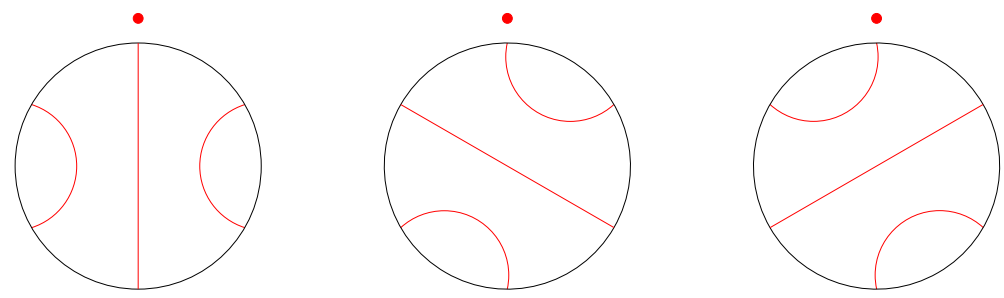

Figure 1: A bypass triple

the lax vectors of Conway [4]. The sum of two lax vectors $\pm v, \pm w$ is not well-defined: choosing representatives $v, w$, we could have $( \pm v)+( \pm w)$ equal to $\pm(v+w)$ or $\pm(v-w)$. The bypass relation then says that three lax vectors $\pm u, \pm v, \pm w$ somehow sum to zero; $\pm(u+v)$ or $\pm(u-v)$ equals $\pm w$.

This paper will resolve these ambiguities, and along the way unearth further structure.

\subsection{Stackability resolves signs}

The key to this resolution is a bilinear form $\langle\cdot \mid \cdot\rangle$ on our abelian group $V$. Over $\mathbb{Z}_{2}$ this was called the stackability map $m(\cdot, \cdot)$ in [17]. Place two chord diagrams as the lids of a cylinder with vertical arcs running along its sides; round corners and arcs in a specific way to obtain a sphere with curves on it; chord diagrams are stackable if we obtain a single connected curve on the sphere. The map

$$
\langle\cdot \mid \cdot\rangle: V \otimes V \longrightarrow \mathbb{Z}
$$

takes stackable chord diagrams to \pm 1 , and nonstackable chord diagrams to 0 ; it reduces to $m(\cdot, \cdot)$ mod 2 . The key idea we shall use to obtain coherent signs for chord diagrams is that while there is ambiguity in \pm 1 , there is no ambiguity in \pm 0 . The bypass relation tells us that a chord diagram should be given by $\pm(u+v)$ or $\pm(u-v)$. However if we know, say, that $\langle u \mid w\rangle=\langle v \mid w\rangle=1$ for some $w$, then $\langle \pm(u-v) \mid w\rangle=0$ while $\langle \pm(u+v) \mid w\rangle= \pm 2$; we can then usefully distinguish between the two.

\subsection{A noncommutative Fock space and partial order}

In quantum field theory a Fock space $\mathcal{F}$ is an algebraic object whose elements can describe states of several particles. For instance, $x \in \mathcal{F}$ can denote the presence of one particle, $y \in \mathcal{F}$ a different particle, and $x y$ the presence of both. A Fock space has creation and annihilation operators, adjoint with respect to an inner product. In a commutative or bosonic (resp. anticommutative or fermionic) Fock space, $x y=y x$ (resp. $x y=-y x$ ). We shall consider a noncommutative $\mathcal{F}$, however, in which neither 
$x y=y x$ nor $x y=-y x$ holds; roughly, $\mathcal{F}$ contains noncommutative polynomials in $x, y$.

There are natural creation and annihilation operators on $\mathcal{F}$, which insert or delete a specified symbol in a word in a specified place - many more creation and annihilation operators than in the commutative or anticommutative cases; they obey the relations of a simplicial set.

In [17] we defined a partial order on words in $\{x, y\}$ (in [17] we used $\{-,+\}$ ). Say that $w_{1} \leq w_{2}$ if $w_{2}$ can be obtained from $w_{1}$ by moving some (possibly none) of the $x$ 's to the right (equivalently, by moving some of the $y$ 's to the left). Thus $x x y y \leq y x y x$ but $x y y x \not \leq y x x y$. Now define a bilinear form $\langle\cdot \mid \cdot\rangle: \mathcal{F} \otimes \mathcal{F} \longrightarrow \mathbb{Z}$ as a boolean version of $\leq$, as follows. For two words $w_{1}, w_{2}$ in $\{x, y\}$, let $\left\langle w_{1} \mid w_{2}\right\rangle=1$ if $w_{1} \leq w_{2}$ and 0 otherwise; then extend to $\mathcal{F}$ linearly. We imagine $\langle\cdot \mid \cdot\rangle$ as a "noncommutative inner product" and $\mathcal{F}$ as a "Fock space of two noncommuting particles". Creation and annihilation operators are adjoint with respect to $\langle\cdot \mid \cdot\rangle$ (in a noncommutative way).

We shall prove the following. (A precise version is Theorem 3.4; a more detailed statement is Theorem 5.1.)

Theorem 1.1 The Fock space and bilinear form defined from $\leq$ are isomorphic to the abelian group $V$ generated by chord diagrams, and bilinear form defined by stacking:

$$
(\mathcal{F},\langle\cdot \mid \cdot\rangle) \cong(V,\langle\cdot \mid \cdot\rangle)
$$

Thus chord diagrams give lax elements of $\mathcal{F}$; three bypass-related chord diagrams give lax elements of $\mathcal{F}$ summing to zero; and stackability is described by the boolean $\leq$. This was shown in [17] $\bmod 2$; we show it holds over $\mathbb{Z}$.

An element of $\mathcal{F}$ corresponding to a chord diagram $\Gamma$ is a linear combination of words in $\{x, y\}$ (up to sign); among these there are well-defined first and last words $w_{-}, w_{+}$. In [17] we showed (over $\left.\mathbb{Z}_{2}\right)$ that $w_{-} \leq w_{+}$; in fact the correspondence $\Gamma \mapsto\left(w_{-}, w_{+}\right)$ gives a bijection between chord diagrams and pairs of words comparable under $\leq$. We will show the same properties also hold over $\mathbb{Z}$; further, every chord diagram gives a linear combination of words with all coefficients \pm 1 .

It is not difficult to show that $\langle\cdot \mid \cdot\rangle$ on $\mathcal{F}$ is nondegenerate. Thus there is a duality operator $H$ such that for all $u, v,\langle u \mid v\rangle=\langle v \mid H u\rangle$. Note that for commutative $\langle\cdot \mid \cdot\rangle$, ie $\langle u \mid v\rangle=\langle v \mid u\rangle$, we have $H=1$; and in the anticommutative case, $H=-1$. We will show that $H$ is periodic, in the sense that some power of $H$ is the identity; this is, in a sense, a generalisation of commutativity and anticommutativity. We prove this statement by showing that $H$ is equivalent to the operation of rotating a chord diagram. 
The key feature distinguishing our Fock space from the usual sort is the form $\langle\cdot \mid \cdot\rangle$, based on $\leq$. Partial orders appear prominently in the theory of causal sets (see eg Bombelli et al [3] and Sorkin [19]), one approach to quantum gravity. But we have not seen anything like $\leq$ in the theoretical physics literature.

\subsection{Sutured Topological Quantum Field Theory}

We have been somewhat vague about $V$. We now explain what $V$ is: a TQFT-like object.

The idea is to build an algebraic structure purely by reference to surfaces (like discs), arcs on them (like chord diagrams), and various topological operations. To a surface $\Sigma$ with some markings $F$ on the boundary, we associate an abelian group $V(\Sigma, F)$. When arcs $\Gamma$ are drawn appropriately on the surface (sutures) we associate an element (up to sign) $c(\Gamma)$ in $V(\Sigma, F)$, called a suture element. These associations are natural with respect to topological operations such as gluing and stacking. This defines what we call sutured topological quantum field theory.

The $V$ above is the sutured TQFT of discs $V\left(D^{2}\right)=\bigoplus_{F} V\left(D^{2}, F\right)$ (direct sum over all possible $F$ ). We impose enough axioms (explaining their rationale as we introduce them) that $V\left(D^{2}\right)$ is unique.

It is interesting to note that although the bypass relation appears in sutured TQFT, we need not impose it as an axiom. Instead we can define $\langle\cdot \mid \cdot\rangle$ from stackability (which is just a certain gluing of surfaces); the bypass relation is equivalent to a nondegeneracy condition on $\langle\cdot \mid \cdot\rangle$ (Proposition 3.7).

The point of this paper is that, in describing sutured TQFT axiomatically, we find surprisingly familiar structures from (nontopological!) quantum field theory, namely all the structure of the Fock space $\mathcal{F}$. The sutured TQFT of discs is the QFT of two noncommuting particles, where the two usages of "QFT" are quite distinct.

We remark that sutured TQFT, as we define it, is very similar in some respects to a planar algebra; see Jones [13]. To a surface with boundary is associated an algebraic object; to curves on the surface, dividing it into positive and negative regions, are associated distinguished elements of the algebraic object; surfaces may be glued together, giving maps of algebraic objects which are natural with respect to the distinguished elements; surfaces with many boundary components give operators. However there are several distinctions: sutured TQFT is not restricted to planar surfaces; sutured TQFT has no canonical form of multiplication (except on discs, where it does not coincide with planar algebra multiplication); the element associated to a set of curves in sutured TQFT has a sign ambiguity; and in the author's limited knowledge, nothing like a bypass relation 
or stackability has been studied in the context of planar algebras. Clearly the two subjects have wildly disparate motivations: planar algebras from subfactors and von Neumann algebras; sutured TQFT from contact geometry and sutured Floer homology. We wonder how deep these similarities run.

\subsection{SFH gives a sutured TQFT}

Although $V$ is defined from sutured TQFT axioms, it is motivated by sutured Floer homology. As discussed by Honda, Kazez and Matić in [10] and at length by the author in [17], the SFH of product manifolds $\left(\Sigma \times S^{1}, F \times S^{1}\right)$, and their contact elements, have properties similar to a $(1+1)$-dimensional TQFT. Sutured TQFT is this TQFT, abstracted from SFH, and now independent of it.

We will show that $\operatorname{SFH}\left(\Sigma \times S^{1}, F \times S^{1}\right)$ forms a sutured TQFT; in particular, for any sutured TQFT, $V\left(D^{2}, F\right) \cong S F H\left(D^{2} \times S^{1}, F \times S^{1}\right)$. In particular, we obtain an explicit description of contact elements, with $\mathbb{Z}$ coefficients, in $S F H\left(D^{2} \times S^{1}, F \times S^{1}\right)$, extending the results over $\mathbb{Z}_{2}$ in [17]. Sutured TQFT on more complicated surfaces is not unique, but we also consider an additional axiom, satisfied by $\operatorname{SFH}\left(\Sigma \times S^{1}, F \times S^{1}\right)$, which ensures nontriviality at higher genus.

This paper thus shows that three structures are equivalent: $\operatorname{SFH}\left(D^{2} \times S^{1}, F \times S^{1}\right)$, $V\left(D^{2}\right)$ and $\mathcal{F}$. Moreover, it shows that all the structure of SFH of product manifolds can be described without considering Heegaard decompositions, holomorphic curves or contact structures. We obtain proofs about contact elements which are both "holomorphic curve free" and "contact geometry free". In a subsequent paper we shall use sutured TQFT to give a proof that the contact element of a torsion contact structure is zero; see Ghiggini et al [6] and Massot [16].

\subsection{Structure of this paper}

Several considerations determine the structure of this paper. To prove our main result that $V\left(D^{2}\right) \cong \mathcal{F}$, it is easiest first to define the formal algebraic structure of $\mathcal{F}$, and then show SFH is isomorphic to it. Moreover, we may eschew sutured Floer homology until the last minute; it is not necessary to the discussion of sutured TQFT or noncommutative QFT, although we do use it to prove that a sutured TQFT exists! In a sense, our results are more general than SFH: they are about suture elements in sutured TQFT, of which contact elements in SFH of product manifolds form an example.

Thus, this paper begins by establishing the formal algebraic structure of $\mathcal{F}$ (Section 2). Much of the detail in this section (especially the bisimplicial structure in Section 2.3, differentials, commutation relations, normal form in Section 2.7 and Temperley-Lieb 
representation in Section 2.8) can be skipped on a first reading: the important details are the Fock space $\mathcal{F}$, the creation and annihilation operations, the bilinear form $\langle\cdot \mid \cdot\rangle$ and the duality operator $H$.

Then in Section 3 we axiomatically introduce sutured TQFT, and deduce properties of it. Some of this is also quite technical (eg variations on axioms) and can be skipped on a first reading. In Section 4 we show that $\operatorname{SFH}\left(\Sigma \times S^{1}, F \times S^{1}\right)$ forms a sutured TQFT, hence that a sutured TQFT exists. In Section 5 we demonstrate $V\left(D^{2}\right) \cong \mathcal{F}$, along with isomorphisms of detailed structures.

Acknowledgements This paper was written during the author's visit to the Mathematical Sciences Research Institute in March 2010, and during the author's postdoctoral fellowship at the Université de Nantes, supported by the ANR grant "Floer power".

\section{Algebraic noncommutative QFT}

\subsection{Fock space}

Let $S=\{x, y\}$ and let $\mathcal{M}$ be the free monoid on $S$, ie the set of all finite words (including the empty word, which is the identity 1) on $\{x, y\}$, under the operation of concatenation. Let $\mathcal{F}$ be the monoid ring of $\mathcal{M}$ over $\mathbb{Z}$. That is, $\mathcal{F}$ consists of finite $\mathbb{Z}$-linear combinations of finite words on $\{x, y\}$; multiplication is concatenation, now extended linearly. Alternatively, $\mathcal{F}$ is the polynomial ring generated over $\mathbb{Z}$ by two noncommuting indeterminates $x, y$. The empty word, denoted 1 , is a multiplicative identity; it is also called the vacuum.

Clearly $\mathcal{M}$ and $\mathcal{F}$ have several gradings: degree $n_{x}$ in $x$, degree $n_{y}$ in $y$, and linear combinations of these. Multiplication adds these gradings, making $\mathcal{F}$ into a bigraded ring. Let $\mathcal{M}_{n}$ denote the subset of $\mathcal{M}$ consisting of words of length $n$, ie with total degree $n$, and $\mathcal{F}_{n} \subset \mathcal{F}$ the additive subgroup generated by $\mathcal{M}_{n}$, ie linear combinations of length- $n$ words. As graded abelian groups, $\mathcal{F}=\bigoplus_{n \geq 0} \mathcal{F}_{n}$ and $\mathcal{F}_{n}=(\mathbb{Z} x \oplus \mathbb{Z} y)^{\otimes n}$. Denote by $\mathcal{M}_{n_{x}, n_{y}}$ the subset of $\mathcal{M}$ consisting of words of degree $n_{x}, n_{y}$ in $x, y$ respectively; and by $\mathcal{F}_{n_{x}, n_{y}} \subset \mathcal{F}$ the additive subgroup generated by $\mathcal{M}_{n_{x}, n_{y}}$.

As an alternative notation, thinking of $x$ as having degree -1 and $y$ having degree 1 , let $\mathcal{M}_{n}^{e}$ denote the subset of $\mathcal{M}$ consisting of words of length $n$ and degree $e$ (ie such that $n_{y}-n_{x}=e$ ), and $\mathcal{F}_{n}^{e}$ the additive subgroup of $\mathcal{F}$ generated by $\mathcal{M}_{n}^{e}$. So $n, e, n_{x}, n_{y}$ are related by

$$
n=n_{x}+n_{y}, \quad e=-n_{x}+n_{y}, \quad n_{x}=\frac{n-e}{2}, \quad n_{y}=\frac{n+e}{2} .
$$


Obviously, $\mathcal{M}_{n_{x}, n_{y}}=\mathcal{M}_{n_{x}+n_{y}}^{n_{y}-n_{x}}, \mathcal{M}_{n}^{e}=\mathcal{M}_{(n-e) / 2, n+e / 2}, \mathcal{F}_{n_{x}, n_{y}}=\mathcal{F}_{n_{x}+n_{y}}^{n_{y}-n_{x}}$ and $\mathcal{F}_{n}^{e}=\mathcal{F}_{n-e / 2, n+e / 2}$. Further,

$$
\mathcal{M}=\bigsqcup_{n} \mathcal{M}_{n}=\bigsqcup_{n, e} \mathcal{M}_{n}^{e}=\bigsqcup_{n_{x}, n_{y}} \mathcal{M}_{n_{x}, n_{y}}, \quad \mathcal{F}=\bigoplus_{n} \mathcal{F}_{n}=\bigoplus_{n, e} \mathcal{F}_{n}^{e}=\bigoplus_{n_{x}, n_{y}} \mathcal{F}_{n_{x}, n_{y}},
$$

where $0 \leq n \in \mathbb{Z}$, and $e \in \mathbb{Z}$ satisfies $|e| \leq n$, and $e \equiv n \bmod 2$. As abelian groups

$$
\mathcal{F}_{n} \cong \mathbb{Z}^{2^{n}} \quad \text { and } \quad \mathcal{F}_{n}^{e} \cong \mathbb{Z}^{\left(\begin{array}{c}
n \\
n x
\end{array}\right)}=\mathbb{Z}^{\left(\begin{array}{c}
n \\
n y
\end{array}\right)}=\mathbb{Z}^{\left(\begin{array}{c}
n \\
(n-e) / 2
\end{array}\right)}=\mathbb{Z}^{\left(\begin{array}{c}
n \\
(n+e) / 2
\end{array}\right)}
$$

\subsection{Creation and annihilation operators}

Operations on words give operations on $\mathcal{F}$. Precisely, any function $\mathcal{M} \longrightarrow \mathcal{M} \cup\{0\}$ extends to a linear operator on $\mathcal{F}$. Thus we define the following operations; $s$ denotes a letter in $\{x, y\}$.

(i) (a) Initial annihilation $a_{s, 0} w$ : if $w$ begins with an $s$, delete it; else return 0 .

(b) Internal annihilation $a_{s, i} w$, for $1 \leq i \leq n_{s} w$ : delete the $i$-th $s$ in $w$.

(c) Final annihilation $a_{s, n_{s} w+1} w$ : if $w$ ends with an $s$, delete it; else return 0 .

(ii) (a) Initial creation $a_{s, 0}^{*} w$ : prepend an $s$ to the beginning of $w$.

(b) Internal creation $a_{s, i}^{*} w$, for $1 \leq i \leq n_{s} w$ : replace the $i-$ th $s$ in $w$ with $s s$.

(c) Final creation $a_{s, n_{s}+1}^{*} w$ : append an $s$ to the end of $w$.

The names initial, internal, and final should be clear; initial and final annihilation (resp. creation) are collectively called terminal annihilation (resp. creation). We then have

$$
\begin{gathered}
a_{x, i}: \mathcal{F}_{n}^{e} \longrightarrow \mathcal{F}_{n-1}^{e+1}, \quad a_{y, i}: \mathcal{F}_{n}^{e} \longrightarrow \mathcal{F}_{n-1}^{e-1}, \\
a_{x, i}^{*}: \mathcal{F}_{n}^{e} \longrightarrow \mathcal{F}_{n+1}^{e-1}, \quad a_{y, i}^{*}: \mathcal{F}_{n}^{e} \longrightarrow \mathcal{F}_{n+1}^{e+1} .
\end{gathered}
$$

Here we take $\mathcal{F}_{-1}^{e}=\{0\}$ : on the vacuum 1 all annihilation operators give 0 .

These operators satisfy various relations which are easily checked, as follows

(i) Between $x$ and $y$; interspecies.

(a) In almost every case, $x$-annihilation/creation and $y$-creation/annihilation commute. That is, for $0 \leq i \leq n_{x}+1$ and $0 \leq j \leq n_{y}+1$, except for $(i, j)=(0,0)$ or $\left(n_{x}+1, n_{y}+1\right)$,

$$
\begin{aligned}
& a_{x, i} \circ a_{y, j}=a_{y, j} \circ a_{x, i}, \quad a_{x, i}^{*} \circ a_{y, j}=a_{y, j} \circ a_{x, i}^{*}, \\
& a_{x, i} \circ a_{y, j}^{*}=a_{y, j}^{*} \circ a_{x, i}, \quad a_{x, i}^{*} \circ a_{y, j}^{*}=a_{y, j}^{*} \circ a_{y, j}^{*} .
\end{aligned}
$$

(b) Initial $x$-annihilation/creation and initial $y$-annihilation/creation never commute.

(c) Final $x$-annihilation/creation and final $y$-annihilation/creation never commute. 
(ii) Among $x$ or among $y$; intraspecies. Set $s$ to be $x$ or $y$.

(a) Annihilations commute after a shift. For $0 \leq i<j \leq n_{s}+1($ not $i=j$ ),

$$
a_{s, i} \circ a_{s, j}=a_{s, j-1} \circ a_{s, i} .
$$

(b) Annihilations and creations usually commute after a shift, and are sometimes inverses. For $0 \leq i, j \leq n_{s}+1$,

$$
a_{s, i} \circ a_{s, j}^{*}= \begin{cases}a_{s, j-1}^{*} \circ a_{s, i} & i<j, \\ 1 & i=j, j+1, \\ a_{s, j}^{*} \circ a_{s, i-1} & i>j+1 .\end{cases}
$$

(c) Creations commute, after a shift. For $0 \leq i \leq j \leq n_{s}+1$,

$$
a_{s, i}^{*} \circ a_{s, j}^{*}=a_{s, j+1}^{*} \circ a_{s, i}^{*} \text {. }
$$

\subsection{Bisimplicial structure}

As noted in [17], the intraspecies relations are those of a simplicial set. We have two simplicial structures on $\mathcal{F}$, one for $x$ and one for $y$. Let $s \in\{x, y\}$. Words with $s$-degree $n_{s}$ have $n_{s}+2$ annihilation operators ( $n_{s}$ internal and 2 terminal), regarded as face maps, and $n_{s}+2$ creation operators, regarded as degeneracy maps; thus words of $s$-degree $n_{s}$ can be regarded as $\left(n_{s}+1\right)$-dimensional simplices.

As a bisimplicial object in the category of abelian groups, $\mathcal{F}$ has contravariant functors

$$
\mathbb{F}_{x}, \mathbb{F}_{y}: \Delta \longrightarrow \mathrm{Ab} \text {. }
$$

Here $\Delta$ is the simplicial category; its objects are the (set-theoretic) nonnegative integers $\mathbf{n}=\{0,1, \ldots, n-1\}$ and its morphisms are order-preserving functions $\mathbf{m} \rightarrow \mathbf{n}$. Ab is the category of abelian groups. These functors have "image $\mathcal{F}$ ", $\mathcal{F}=\bigoplus_{n} \mathbb{F}_{x}(\mathbf{n})=$ $\bigoplus_{n} \mathbb{F}_{y}(\mathbf{n})$, as we now describe.

In fact the monoid $\mathcal{M}$ also has a bisimplicial structure, arising from functors

$$
\mathbb{F}_{x}, \mathbb{F}_{y}: \Delta \longrightarrow \text { Set, }
$$

with $\bigsqcup_{n} \mathbb{F}_{x}(\mathbf{n})=\bigsqcup_{n} \mathbb{F}_{y}(\mathbf{n})=\mathcal{M} \cup\{0\}$. (We adjoin 0 since terminal annihilations may return 0 .) The functors to Set for $\mathcal{M}$ extend linearly to the functors to $\mathrm{Ab}$, as the elements of $\mathcal{M}$ form a basis for $\mathcal{F}$.

The functors $\mathbb{F}_{s}$ can be described by regarding a word $w$ on $\{x, y\}$ as a function. It will be useful to describe words by functions in several different ways, and we now pause to describe these functions. 
Definition 2.1 Let $w \in \mathcal{M}_{n_{x}, n_{y}}, n=n_{x}+n_{y}$ and number the letters in $w$ left to right from $x_{1}$ to $x_{n_{x}}$ and $y_{1}$ to $y_{n_{y}}$. Define functions $f_{w}^{x}, f_{w}^{y}, g_{w}^{x}, g_{w}^{y}, h_{w}^{x}, h_{w}^{y}$ as follows.

(i) $f_{w}^{x}:\left\{1,2, \ldots, n_{x}\right\} \longrightarrow\left\{0,1, \ldots, n_{y}\right\}, f_{w}^{x}(i)$ is the number of $y$ 's (strictly) to the left of $x_{i}$ in $w$.

(ii) $f_{w}^{y}:\left\{1,2, \ldots, n_{y}\right\} \longrightarrow\left\{0,1, \ldots, n_{x}\right\}, f_{w}^{y}(i)$ is the number of $x$ 's (strictly) to the left of $y_{i}$ in $w$.

(iii) $g_{w}^{x}:\{1,2, \ldots, n\} \longrightarrow\left\{0,1, \ldots, n_{x}\right\}, g_{w}^{x}(i)$ is the number of $x$ 's in the first $i$ letters of $w$.

(iv) $g_{w}^{y}:\{1,2, \ldots, n\} \longrightarrow\left\{0,1, \ldots, n_{y}\right\}, g_{w}^{y}(i)$ is the number of $y$ 's in the first $i$ letters of $w$.

(v) $h_{w}^{x}:\left\{1,2, \ldots, n_{x}\right\} \longrightarrow\{1,2, \ldots, n\}, h_{w}^{x}(i)$ is the position of $x_{i}$ in $w$.

(vi) $h_{w}^{y}:\left\{1,2, \ldots, n_{y}\right\} \longrightarrow\{1,2, \ldots, n\}, h_{w}^{y}(i)$ is the position of $y_{i}$ in $w$.

Clearly the $f_{w}^{s}$ are increasing. The $g_{w}^{s}$ are slowly increasing, $g_{w}^{s}(i+1)-g_{w}^{s}(i) \in\{0,1\}$, and $g_{w}^{s}(1) \in\{0,1\}, g_{w}^{s}\left(n_{x}+n_{y}\right)=n_{s}$. (Note $g_{w}^{s}(i)$ is like the baseball team's score after $i$ innings of [17].) The $h_{w}^{s}$ are strictly increasing. It's clear that any of these functions corresponds to a unique word $w$; moreover, there is a bijection between $\mathcal{M}_{n_{x}, n_{y}}$ and functions with these respective properties. So one of these functions determines all the others: for instance, $h_{w}^{s}(i)=f_{w}^{s}(i)+i$; the images of $h_{w}^{x}$ and $h_{w}^{y}$ form a partition of $\{1, \ldots, n\}$; and $h_{w}^{s}(i)=\min \left\{\left(g_{w}^{s}\right)^{-1}(i)\right\}$; also $g_{w}^{x}(i)+g_{w}^{y}(i)=i$.

For the moment we only need the $f_{w}^{s}$. As the $f_{w}^{s}$ are increasing (order-preserving), they can be regarded as morphisms in $\Delta$; after a shift, we may regard $f_{w}^{x} \in \operatorname{Mor}_{\Delta}\left(\mathbf{n}_{\mathbf{x}}, \mathbf{n}_{\mathbf{y}}+\mathbf{1}\right)$; so $\mathcal{M}_{n_{x}, n_{y}}$ and $\operatorname{Mor}_{\Delta}\left(\mathbf{n}_{\mathbf{x}}, \mathbf{n}_{\mathbf{y}}+\mathbf{1}\right)$ are bijective.

The idea of $\mathbb{F}_{x}$ is to take each object $\mathbf{n}$ in $\Delta$ to the set of all words in $\mathcal{M}$ with degree $n$ in $x$, which is $\bigsqcup_{n_{y}} \mathcal{M}_{n_{x}, n_{y}} \cong \bigsqcup_{n_{y}} \operatorname{Mor}_{\Delta}\left(\mathbf{n}, \mathbf{n}_{\mathbf{y}}+\mathbf{1}\right)$; we can denote this $\operatorname{Mor}_{\Delta}(\mathbf{n}, \cdot)$. Then $\mathbb{F}_{x}$ takes the morphism $g: \mathbf{m} \rightarrow \mathbf{n}$ of $\Delta$ to the function

$$
\operatorname{Mor}_{\Delta}(\mathbf{n}, \cdot) \stackrel{\mathbb{F}_{x} g}{\longrightarrow} \operatorname{Mor}_{\Delta}(\mathbf{m}, \cdot)
$$

that is given by precomposition with $g$. Note $\mathbb{F}_{x}(g)$ takes each $\operatorname{Mor}_{\Delta}\left(\mathbf{n}, \mathbf{n}_{\mathbf{y}}+\mathbf{1}\right)$ to $\operatorname{Mor}_{\Delta}\left(\mathbf{m}, \mathbf{n}_{\mathbf{y}}+\mathbf{1}\right)$, hence preserves $n_{y}$ (which makes sense as this is the structure of adding and deleting $x$ 's).

However this structure does not allow for terminal creation and annihilation operators. The idea is to modify the above description by appending and prepending an $x$ to the beginning and end of each word. Define the subset $\operatorname{Mor}_{\Delta}^{T}(\mathbf{m}, \mathbf{n})$ of $\operatorname{Mor}_{\Delta}(\mathbf{m}, \mathbf{n})$ to be those order-preserving maps $\mathbf{m} \rightarrow \mathbf{n}$ which take $0 \mapsto 0$ and 
$m-1 \mapsto n-1$; call them terminal-preserving morphisms. There is a natural bijection $\operatorname{Mor}_{\Delta}(\mathbf{m}, \mathbf{n}) \cong \operatorname{Mor}_{\Delta}^{T}(\mathbf{m}+\mathbf{2}, \mathbf{n})$ given by shifting a map by one and setting its values on 0 and $m+1$. The composition of a morphism in $\operatorname{Mor}_{\Delta}^{T}(\mathbf{m}, \mathbf{n})$ and a morphism in $\operatorname{Mor}_{\Delta}^{T}(\mathbf{n}, \mathbf{k})$ is a morphism in $\operatorname{Mor}_{\Delta}^{T}(\mathbf{m}, \mathbf{k})$ : terminal-preserving morphisms are closed under composition.

Words of degree $n_{x}, n_{y}$ in $x, y$ are in bijective correspondence with words of degree $n_{x}+2, n_{y}$ in $x, y$ which begin and end with $x$. Similarly, increasing functions $\mathbf{n}_{\mathbf{x}} \rightarrow \mathbf{n}_{\mathbf{y}}+\mathbf{1}$ are in bijective correspondence with $\operatorname{Mor}_{\Delta}^{T}\left(\mathbf{n}_{\mathbf{x}}+\mathbf{2}, \mathbf{n}_{\mathbf{y}}+\mathbf{1}\right)$. We define $\mathbb{F}_{x}$ to take $\mathbf{n} \in \mathrm{Ob} \Delta$ to the set of all words of degree $n-2$ in $x$, along with 0 . That is,

$$
\begin{aligned}
\mathbb{F}_{x}(\mathbf{n}) & =\{0\} \cup\{\text { words of } x \text {-degree } n-2 \text { in } \mathcal{M}\} \\
& \cong\{0\} \cup\left\{\begin{array}{c}
\text { words of } x \text {-degree } n \text { in } \mathcal{M} \\
\text { which begin and end with } x
\end{array}\right\} \\
& \cong\{0\} \cup \bigsqcup_{n_{y}} \operatorname{Mor}_{\Delta}\left(\mathbf{n}-\mathbf{2}, \mathbf{n}_{\mathbf{y}}+\mathbf{1}\right) \\
& \cong\{0\} \cup \bigsqcup_{n_{y}} \operatorname{Mor}_{\Delta}^{T}\left(\mathbf{n}, \mathbf{n}_{\mathbf{y}}+\mathbf{1}\right) \cong\{0\} \cup \operatorname{Mor}_{\Delta}^{T}(\mathbf{n}, \cdot)
\end{aligned}
$$

For $n=0,1$ then $\mathbb{F}_{x}(\mathbf{n})=\{0\}$. We define $\mathbb{F}_{x}$ to take a morphism $g \in \operatorname{Mor}_{\Delta}(\mathbf{m}, \mathbf{n})$ to the function

$$
\{0\} \cup \operatorname{Mor}_{\Delta}^{T}(\mathbf{n}, \cdot) \stackrel{\mathbb{F}_{x} g}{\longrightarrow}\{0\} \cup \operatorname{Mor}_{\Delta}^{T}(\mathbf{m}, \cdot)
$$

which takes $0 \mapsto 0$ and which precomposes functions by $g$, if such precomposition gives a terminal-preserving morphism; else gives 0 . (If $g$ is terminal-preserving then such a composition is certainly terminal-preserving; if $g$ is not terminal-preserving then the composition may or may not be terminal-preserving.) This $\mathbb{F}_{x} g$ preserves $y$-degree unless it maps to 0 .

This gives the contravariant functor $\mathbb{F}_{x}: \Delta \longrightarrow$ Set with image $\mathcal{M}$, which extends to $\mathbb{F}_{x}: \Delta \longrightarrow$ Ab with image $\mathcal{F}$.

For each $n_{x} \geq 0$ and $0 \leq i \leq n_{x}+1$, define $a_{x, i}$ to be the unique morphism in $\operatorname{Mor}_{\Delta}\left(\mathbf{n}_{\mathbf{x}}+\mathbf{1}, \mathbf{n}_{\mathbf{x}}+\mathbf{2}\right)$ which has image $\left(\mathbf{n}_{\mathbf{x}}+\mathbf{2}\right) \backslash\{i\}$, in other words, which takes $\left(0,1, \ldots, i-1, i, \ldots, n_{x}\right) \mapsto\left(0,1, \ldots, i-1, i+1, \ldots, n_{x}+1\right)$. Such an $a_{x, i}$, under $\mathbb{F}_{x}$, gives a map $\mathbb{F}_{x}\left(\mathbf{n}_{\mathbf{x}}+\mathbf{2}\right) \rightarrow \mathbb{F}_{x}\left(\mathbf{n}_{\mathbf{x}}+\mathbf{1}\right)$, ie

$\{0\} \cup\left\{\right.$ words of $x$-degree $n_{x}$ in $\left.\mathcal{M}\right\} \longrightarrow\{0\} \cup\left\{\right.$ words of $x$-degree $n_{x}-1$ in $\left.\mathcal{M}\right\}$,

which is seen to be $a_{x, i}$ as originally defined. Note that the terminal annihilation operators $a_{x, 0}, a_{x, n_{x}+1}$ are not terminal-preserving (rather "terminal-annihilating") 
but all other $a_{x, i}$ are terminal-preserving; hence $a_{x, 0}, a_{x, n_{x}+1}$ sometimes return zero, but other $a_{x, i}$ do not.

Similarly, for each $n_{x} \geq 0$ and $0 \leq i \leq n_{x}+1$, let $a_{x, i}^{*}$ be the unique surjective morphism in $\operatorname{Mor}_{\Delta}\left(\mathbf{n}_{\mathbf{x}}+\mathbf{3}, \mathbf{n}_{\mathbf{x}}+\mathbf{2}\right)$ taking the value $i$ twice, ie $\left(0,1, \ldots, i, i+1, \ldots, n_{x}+2\right) \mapsto$ $\left(0,1, \ldots, i, i, \ldots, n_{x}+1\right)$. Then $\mathbb{F}_{x}\left(a_{x, i}^{*}\right): \mathbb{F}_{x}\left(\mathbf{n}_{\mathbf{x}}+\mathbf{2}\right) \rightarrow \mathbb{F}_{x}\left(\mathbf{n}_{\mathbf{x}}+\mathbf{3}\right)$ is a map $\{0\} \cup\left\{\right.$ words of $x$-degree $n_{x}$ in $\left.\mathcal{M}\right\} \longrightarrow\{0\} \cup\left\{\right.$ words of $x$-degree $n_{x}+1$ in $\left.\mathcal{M}\right\}$ which is $a_{x, i}^{*}$ as originally defined.

The functor $\mathbb{F}_{y}$ is defined similarly, using $f_{w}^{y}$. This gives two "dual" simplicial structures.

\subsection{Partial order}

Inequalities on all the functions $f_{w}^{s}, g_{w}^{s}, h_{w}^{s}$ are equivalent, in the following sense.

Lemma 2.2 Let $w_{0}, w_{1} \in \mathcal{M}_{n_{x}, n_{y}}$. The following inequalities are all equivalent: $f_{w_{0}}^{x} \leq f_{w_{1}}^{x}, \quad f_{w_{0}}^{y} \geq f_{w_{1}}^{y}, \quad g_{w_{0}}^{x} \geq g_{w_{1}}^{x}, \quad g_{w_{0}}^{y} \leq f_{w_{1}}^{y}, \quad h_{w_{0}}^{x} \leq h_{w_{1}}^{x}, \quad h_{w_{0}}^{y} \geq h_{w_{1}}^{y}$.

Proof The inequalities on $f_{w_{i}}^{s}$ and $h_{w_{i}}^{s}$ are clearly equivalent, since $h_{w}^{s}(i)=f_{w}^{s}(i)+i$. The inequalities on $g_{w_{i}}^{s}$ and $h_{w_{i}}^{s}$ are equivalent since the $g_{w_{i}}^{s}$ are slowly increasing and $h_{w}^{s}(i)=\min \left\{\left(g_{w}^{s}\right)^{-1}(i)\right\}$. The inequalities on $h_{w_{i}}^{x}$ and $h_{w_{i}}^{y}$ are equivalent since their images are complementary.

If these inequalities hold, we say $w_{0} \leq w_{1}$. It's clear this gives a partial order on each $\mathcal{M}_{n_{x}, n_{y}}$, which is a suborder of the lexicographic (total) ordering ( $x$ comes before $y$ ). Words with different $x$ - or $y$-degrees are not comparable.

If $h$ is order-preserving and $f \leq g$ then $h \circ f \leq h \circ g$. Because the creations $a_{s, i}^{*}$ are order-preserving $\left(\mathbf{n}_{\mathbf{s}}+\mathbf{3} \rightarrow \mathbf{n}_{\mathbf{s}}+\mathbf{2}\right.$, under $\mathbb{F}_{s}$ mapping $\operatorname{Mor}_{\Delta}^{T}\left(\mathbf{n}_{\mathbf{s}}+\mathbf{2}, \cdot\right) \rightarrow$ $\operatorname{Mor}_{\Delta}^{T}\left(\mathbf{n}_{\mathbf{s}}+\mathbf{3}, \cdot\right)$ by precomposition $)$,

$$
w_{0} \leq w_{1} \quad \text { implies } \quad a_{s, i}^{*} w_{0} \leq a_{s, i}^{*} w_{1}
$$

and in fact the converse is also true.

The same is not true for annihilation operators. Terminal annihilations may map to zero, so that no comparison is possible. Even when nonzero we may have $w_{0} \not \leq w_{1}$ but $a_{s, i} w_{0} \leq a_{s, i} w_{1}$; eg $y x x y \not \leq x y y x$ but, applying $a_{1, x}$ to both sides, $y x y \leq y y x$. It is however true that if $w_{0} \leq w_{1}$ and $a_{s, i} w_{0} \neq 0, a_{s, i} w_{1} \neq 0$ then $a_{s, i} w_{0} \leq a_{s, i} w_{1}$. Later on (Section 3.13) we shall examine this partial order in more detail; we will need notions of difference between words, and minimum and maximum of two words. 
Definition 2.3 For two words $w_{0}, w_{1} \in \mathcal{M}_{n_{x}, n_{y}}$ (comparable or not), their difference is

$$
d\left(w_{0}, w_{1}\right)=\sum_{i=1}^{n_{x}} h_{w_{1}}^{x}(i)-h_{w_{0}}^{x}(i) .
$$

As $h_{w}^{x}(i)$ is the position of the $i-$ th $x$ in $w, h_{w_{1}}^{x}(i)-h_{w_{0}}^{x}(i)$ gives the difference in position between the $i$-th $x$ 's in $w_{0}$ and $w_{1}$. If we regard $x$ 's as pawns and $y$ 's as empty squares on a $1 \times n$ chessboard, then $d\left(w_{0}, w_{1}\right)$ is the number of signed (rightwards $=$ positive) pawn moves required to go from $w_{0}$ to $w_{1}$. If $w_{0} \leq w_{1}$, then only rightwards moves are required and $d\left(w_{0}, w_{1}\right)$ is the number of such moves.

Lemma 2.4 For any $w_{0}, w_{1} \in \mathcal{M}_{n_{x}, n_{y}}$ (comparable or not), there are decompositions

$$
w_{0}=w_{0}^{0} w_{0}^{1} \cdots w_{0}^{2 k-1}, \quad w_{1}=w_{1}^{0} w_{1}^{1} \cdots w_{1}^{2 k-1},
$$

where $w_{0}^{i} \leq w_{1}^{i}$ if $i$ is even and $w_{1}^{i} \leq w_{0}^{i}$ if $i$ is odd. The words $w_{0}^{0}, w_{1}^{0}, w_{0}^{2 k-1}, w_{1}^{2 k-1}$ might be empty, but the other $w_{j}^{i}$ are not. (In particular, each $w_{0}^{i}, w_{1}^{i}$ have equal $x-$ and $y$-degrees.)

Proof Consider the baseball interpretation of [17]: when scores are level, split the words $w_{0}, w_{1}$; once on each interval of the game in which scores remain level. Any baseball game splits into subgames on which the leading team alternates, giving the desired inequality.

Having done this, let

$$
w_{-}=w_{0}^{0} w_{1}^{1} w_{0}^{2} w_{1}^{3} \cdots w_{1}^{2 k-1}, \quad w_{+}=w_{1}^{0} w_{0}^{1} w_{1}^{2} w_{0}^{3} \cdots w_{0}^{2 k-1} .
$$

One can easily verify

$$
\begin{array}{lll}
f_{w_{-}}^{x}=\min \left(f_{w_{0}}^{x}, f_{w_{1}}^{x}\right), & g_{w_{-}}^{y}=\min \left(g_{w_{0}}^{y}, g_{w_{1}}^{y}\right), & h_{w_{-}}^{x}=\min \left(h_{w_{0}}^{x}, h_{w_{1}}^{x}\right), \\
f_{w_{+}}^{x}=\max \left(f_{w_{0}}^{x}, f_{w_{1}}^{x}\right), & g_{w_{+}}^{y}=\max \left(g_{w_{0}}^{y}, g_{w_{1}}^{y}\right), & h_{w_{+}}^{x}=\max \left(h_{w_{0}}^{x}, h_{w_{1}}^{x}\right),
\end{array}
$$

etc. In particular, $w_{-} \leq w_{+}$and, although there might be many ways to split $w_{0}, w_{1}$ (baseball can remain tied for several innings), the resulting $w_{-}, w_{+}$are unique. Call $w_{-}$the minimum and $w_{+}$the maximum of the pair $\left(w_{0}, w_{1}\right)$. If $w_{0} \leq w_{1}$ then $\min \left(w_{0}, w_{1}\right)=w_{0}$ and $\max \left(w_{0}, w_{1}\right)=w_{1}$.

\subsection{Adjoints, bilinear form}

The notation $a_{s, i}, a_{s, i}^{*}$ for annihilation and creation operators suggests that they should be adjoint. They are indeed adjoint with respect to $\leq$, but only in one direction each. We have

$$
a_{y, i}^{*} w_{0} \leq w_{1} \quad \text { if and only if } \quad w_{0} \leq a_{y, i} w_{1}
$$


for $0 \leq i \leq n_{y}\left(w_{1}\right)$, the $y$-degree of $w_{1}$ (note $w_{1}$ has $y$-degree one larger than that of $\left.w_{0}\right)$. Also,

$$
a_{x, i} w_{0} \leq w_{1} \quad \text { if and only if } w_{0} \leq a_{x, i}^{*} w_{1}
$$

for $0 \leq i \leq n_{x}\left(w_{0}\right)$ (here $w_{0}$ has $x$-degree one larger than that of $\left.w_{1}\right)$. These inequalities hold even for terminal creations and annihilations: if a terminal annihilation gives 0 , the inequality is false.

These "adjoint inequalities" are not true in the other direction. For instance, it is not true that $a_{y, i} w_{0} \leq w_{1}$ if and only if $w_{0} \leq a_{y, i}^{*} w_{1}$, since $x x y=a_{y, 1} y x x y \leq x y x$ but $y x x y \not \leq a_{y, 1}^{*} x y x=x y y x$.

As mentioned in the introduction, define a "booleanization" $\langle\cdot \mid \cdot\rangle: \mathcal{M} \times \mathcal{M} \longrightarrow\{0,1\}$. For words $w_{0}, w_{1} \in \mathcal{M},\left\langle w_{0} \mid w_{1}\right\rangle=1$ if and only if $w_{0} \leq w_{1}$; otherwise $\left\langle w_{0} \mid w_{1}\right\rangle=0$. We then extend linearly to $\langle\cdot \mid \cdot\rangle: \mathcal{F} \otimes \mathcal{F} \longrightarrow \mathbb{Z}$. The following properties of $\langle\cdot \mid \cdot\rangle$ are easy to verify.

- It is bilinear over $\mathbb{Z}$ (by definition).

- It is not symmetric, indeed far from it: if $\left\langle w_{0} \mid w_{1}\right\rangle=\left\langle w_{1} \mid w_{0}\right\rangle=1$ then $w_{0}=w_{1}$.

- "Words have norm one": $\langle w \mid w\rangle=1$.

- The decomposition $\mathcal{F}=\bigoplus_{n_{x}, n_{y}} \mathcal{F}_{n_{x}, n_{y}}$ is orthogonal with respect to $\langle\cdot \mid \cdot\rangle$ : if $w_{0}, w_{1}$ have different $x$ - or $y$-degree then $\left\langle w_{0} \mid w_{1}\right\rangle=0$.

- Creation and annihilation operators $a_{s, i}, a_{s, i}^{*}$ are partially adjoint,

$$
\left\langle a_{x, i} w_{0} \mid w_{1}\right\rangle=\left\langle w_{0} \mid a_{x, i}^{*} w_{1}\right\rangle \text { and }\left\langle w_{0} \mid a_{y, i} w_{1}\right\rangle=\left\langle a_{y, i}^{*} w_{0} \mid w_{1}\right\rangle,
$$

for $0 \leq i \leq n_{x}\left(w_{0}\right), 0 \leq i \leq n_{y}\left(w_{1}\right)$ respectively.

- "Creation operators are isometries" (but annihilation operators are not):

$$
\left\langle w_{0} \mid w_{1}\right\rangle=\left\langle a_{s, i}^{*} w_{0} \mid a_{s, i}^{*} w_{1}\right\rangle .
$$

- "Multiplicativity": if $(a, c) \in \mathcal{M}_{n_{x}, n_{y}}$ and $(b, d) \in \mathcal{M}_{n_{x}^{\prime}, n_{y}^{\prime}}$, then $\langle a b \mid c d\rangle=$ $\langle a \mid c\rangle\langle b \mid d\rangle$.

The bilinear form $\langle\cdot \mid \cdot\rangle$ is also nondegenerate. Suppose $\langle v \mid \cdot\rangle=0$, for some $v \neq 0$, $v=\sum_{i} a_{i} w_{i}$, where $w_{i}$ are distinct words and $0 \neq a_{i} \in \mathbb{Z}$. By orthogonality of each $\mathcal{F}_{n_{x}, n_{y}}$ we may assume all $w_{i}$ have the same $x$ - and $y$-degree. Let $w_{-}$be the lexicographically first among the $w_{i}$; then we have $\left\langle w_{-} \mid w_{-}\right\rangle=1$ but for every other $w_{i},\left\langle w_{i} \mid w_{-}\right\rangle=0$. Thus $0=\left\langle v \mid w_{-}\right\rangle=\left\langle a_{-} w_{-} \mid w_{-}\right\rangle=a_{-} \neq 0$, a contradiction. Similarly if $\langle\cdot \mid v\rangle=0$ then by taking $w_{+}$, the lexicographically last among the $w_{i}$, we obtain $\left\langle w_{+} \mid v\right\rangle \neq 0$, a contradiction. This gives nondegeneracy.

In fact, the bilinear form $\langle\mid\rangle$ is essentially unique, in the following sense. 
Proposition 2.5 Suppose $B: \mathcal{F} \otimes \mathcal{F} \longrightarrow \mathbb{Z}$ is a bilinear form such that:

(i) Distinct $\mathcal{F}_{n}^{e}$ are orthogonal: if $w_{0} \in \mathcal{M}_{n_{0}}^{e_{0}}, w_{1} \in \mathcal{M}_{n_{1}}^{e_{1}},\left(n_{0}, e_{0}\right) \neq\left(n_{1}, e_{1}\right)$, then $B\left(w_{0}, w_{1}\right)=0$.

(ii) Creations and annihilations are partially adjoint: $B\left(a_{x, i} w_{0}, w_{1}\right)=B\left(w_{0}, a_{x, i}^{*} w_{1}\right)$ for $0 \leq i \leq n_{x}\left(w_{0}\right)$ and $B\left(w_{0}, a_{y, i} w_{1}\right)=B\left(a_{y, i}^{*} w_{0}, w_{1}\right)$ for $0 \leq i \leq n_{y}\left(w_{1}\right)$.

(iii) $B(1,1)=1$.

Then $B=\langle\cdot \mid \cdot\rangle$.

Proof First note that condition (ii) implies that creation operators are isometries:

$$
\begin{gathered}
B\left(a_{x, i}^{*} w_{0}, a_{x, i}^{*} w_{1}\right)=B\left(a_{x, i} a_{x, i}^{*} w_{0}, w_{1}\right)=B\left(w_{0}, w_{1}\right), \\
B\left(a_{y, i}^{*} w_{0}, a_{y, i}^{*} w_{1}\right)=B\left(w_{0}, a_{y, i} a_{y, i}^{*} w_{1}\right)=B\left(w_{0}, w_{1}\right) .
\end{gathered}
$$

Given any two words $w_{0}, w_{1} \in \mathcal{M}_{n}^{e}$, we note that $B\left(w_{0}, w_{1}\right)$ can be simplified if $w_{0}$ has a repeated $y$ or if $w_{1}$ has a repeated $x$, since then $w_{0}=a_{y, i}^{*} w_{0}^{\prime}$ or $w_{1}=a_{x, i}^{*} w_{1}^{\prime}$; so suppose there are no such repeated symbols. If $w_{0}, w_{1}$ begin with the same symbol then we may simplify, since then $w_{0}=a_{y, 0}^{*} w_{0}^{\prime}$ or $w_{1}=a_{x, 0}^{*} w_{1}^{\prime}$; so suppose they do not. If $w_{0}$ begins with $y$ and $w_{1}$ begins with $x$, then let $w_{1}=a_{x, 0}^{*} w_{1}^{\prime}$ so that $B\left(w_{0}, w_{1}\right)=B\left(w_{0}, a_{x, 0}^{*} w_{1}^{\prime}\right)=B\left(a_{x, 0} w_{0}, w_{1}^{\prime}\right)=0$. Thus we may simplify to the case where $w_{0}$ begins with $x$ and has no repeated $y$; and $w_{1}$ begins with $y$ and has no repeated $x$; but $w_{0}, w_{1}$ lie in the same $\mathcal{M}_{n}^{e}$.

Thus $w_{0}$ has $e \leq 0$; and $w_{1}$ has $e \geq 0$; lying in the same $\mathcal{M}_{n}^{e}$, we must have $e=0$, and in fact $w_{0}=(x y)^{m}, w_{1}=(y x)^{m}$. We now compute, for $m \geq 1$ :

$$
\begin{aligned}
B\left((x y)^{m},(y x)^{m}\right) & =B\left((x y)^{m} y,(y x)^{m} y\right) \\
& =B\left(a_{y, m}^{*}(x y)^{m},(y x)^{m} y\right) \\
& =B\left((x y)^{m}, a_{y, m}(y x)^{m} y\right) \\
& =B\left((x y)^{m},(y x)^{m-1} x y\right)=B\left((x y)^{m-1},(y x)^{m-1}\right) .
\end{aligned}
$$

(In the first line we apply a terminal creation. In the second line we use adjoint relations to reorder some symbols. In the third line we remove terminal creations.) Applying this repeatedly we have $B\left((x y)^{m},(y x)^{m}\right)=B(1,1)=1$, and hence $B=\langle\cdot \mid \cdot\rangle$.

We remark that, since the "stackability map" of [17], mod 2, easily satisfies the hypotheses of this proposition, this gives another proof of the result, proved directly in [17], that the stackability map mod 2 is the boolean version of $\leq$. 


\subsection{Duality}

By nondegeneracy, the bilinear form $\langle\cdot \mid \cdot\rangle$ gives a duality map on each $\mathcal{F}_{n_{x}, n_{y}}$, which is an isomorphism, at least over the rationals. In fact, as $\langle\cdot \mid \cdot\rangle$ is asymmetric, there are two such maps, which are isomorphisms over the rationals. (We will shortly see that these are also isomorphisms over $\mathbb{Z}$.)

$$
\begin{aligned}
& \iota_{-}: \mathcal{F}_{n_{x}, n_{y}} \stackrel{\cong}{\longrightarrow}\left(\mathcal{F}_{n_{x}, n_{y}}\right)^{*}, \quad v \mapsto\langle v \mid \cdot\rangle \\
& \iota_{+}: \mathcal{F}_{n_{x}, n_{y}} \stackrel{\cong}{\longrightarrow}\left(\mathcal{F}_{n_{x}, n_{y}}\right)^{*}, \quad v \mapsto\langle\cdot \mid v\rangle
\end{aligned}
$$

Composing these two maps in the two possible directions gives two inverse automorphisms of each $\mathcal{F}_{n}^{e}$ :

$$
\begin{aligned}
H & =\iota_{+}^{-1} \circ \iota_{-}: \mathcal{F}_{n}^{e} \longrightarrow \mathcal{F}_{n}^{e}, \\
H^{-1} & =\iota_{-}^{-1} \circ \iota_{+}: \mathcal{F}_{n}^{e} \longrightarrow \mathcal{F}_{n}^{e} .
\end{aligned}
$$

It's clear from the definition that $\langle u \mid v\rangle=\langle v \mid H u\rangle=\left\langle H^{-1} v \mid u\right\rangle$. Thus $H$ is "unitary" with respect to $\langle\cdot \mid \cdot\rangle, H^{*}=H^{-1} ; H$ is an isometry, $\langle u \mid v\rangle=\langle H u \mid H v\rangle$.

We will show in Section 5.4 that $H$ is periodic; we know no direct algebraic proof of this result.

Theorem 2.6 On $\mathcal{F}_{n_{x}, n_{y}}, H^{n+1}=(-1)^{n_{x} n_{y}}$. In particular $H^{2 n+2}=1$. The period of $H$ is $2 n+2$, if both $n_{x}, n_{y}$ are odd; else the period is $n+1$.

There is another bilinear form on $\mathcal{F}$, which we denote by a dot $\cdot$, with respect to which the basis $\mathcal{M}$ of words is orthonormal, ie $w_{0} \cdot w_{1}=1$ if $w_{0}=w_{1}$ and $w_{0}$. $w_{1}=0$ otherwise. This $\cdot$ is clearly symmetric, $\mathbb{Z}$-bilinear, and nondegenerate. By nondegeneracy of both bilinear forms $\langle\cdot \mid \cdot\rangle$ and $\cdot$, it follows that there are operators $Q_{+}, Q_{-}: \mathcal{F}_{n}^{e} \longrightarrow \mathcal{F}_{n}^{e}$, over $\mathbb{Q}$, which are isomorphisms over $\mathbb{Q}$, intertwining the two forms, ie such that for all $u, v \in \mathcal{F}_{n}^{e}$,

$$
u \cdot v=\left\langle u \mid Q_{+} v\right\rangle=\left\langle Q_{-} u \mid v\right\rangle .
$$

But since $\cdot$ is symmetric, we have also

$$
u \cdot v=v \cdot u=\left\langle v \mid Q_{+} u\right\rangle=\left\langle Q_{-} v \mid u\right\rangle=\left\langle H^{-1} Q_{+} u \mid v\right\rangle=\left\langle u \mid H Q_{-} v\right\rangle .
$$

It follows that

$$
H=Q_{+} Q_{-}^{-1} \text {. }
$$

Reading a word $w$ from left to right, an $x$ which is immediately followed by a $y$ is called an exceptional $x ; E_{w}^{x}$ denotes the set of exceptional $x$ 's in $w$. For every subset $T \subseteq E_{w}^{x}$, let $\psi_{T}^{x} w$ denote the operation of taking each $x$ in $T$, and its immediately following $y$, and replacing this $x y$ with $y x$, ie "moving the $x$ forwards one position". 
For example, if $w=x_{1} x_{2} y_{1} y_{2} x_{3} y_{3}$ (we use subscripts to distinguish letters), then $E_{w}^{x}=\left\{x_{2}, x_{3}\right\}$, and if $T=\left\{x_{2}\right\}$ then $\psi_{T}^{x} w=x_{1} y_{1} x_{2} y_{2} x_{3} y_{3}$. Similarly $E_{w}^{y}$ denotes the set of exceptional $y$ 's in $w$, ie those which are followed by an $x$; and for $T \subseteq E_{w}^{y}$, $\psi_{T}^{y} w$ denotes the operation of taking each $y$ in $T$, and its following $x$, and replacing this $y x$ with $x y$, "moving the $y$ forwards one position". If our word is a onedimensional chessboard, $x$ 's are pawns, and $y$ 's are empty squares, then $\psi^{x}$ advances pawns to the right, and $\psi^{y}$ advances pawns to the left.

We have the following explicit formulas for $Q_{ \pm}^{ \pm 1}$.

Proposition 2.7 For any word $w \in \mathcal{M}_{n}^{e}$,

$$
\begin{aligned}
& Q_{+} w=\sum_{T \subseteq E_{w}^{y}}(-1)^{|T|} \psi_{T}^{y} w, \quad Q_{+}^{-1} w=\sum_{w_{i} \leq w} w_{i}, \\
& Q_{-} w=\sum_{T \subseteq E_{w}^{x}}(-1)^{|T|} \psi_{T}^{x} w, \quad Q_{-}^{-1} w=\sum_{w_{i} \geq w} w_{i} .
\end{aligned}
$$

(So, for example, if $w=x y x y$ then $Q_{-} w=x y x y-x y y x-y x x y+y x y x$ and $Q_{+} w=x y x y-x x y y$.)

Proof First, consider $Q_{+}^{-1}$. For any words $w_{0}, w_{1} \in \mathcal{M}_{n}^{e} \subset \mathcal{F}_{n}^{e}$ we have

$$
w_{0} \cdot Q_{+}^{-1} w_{1}=\left\langle w_{0} \mid w_{1}\right\rangle= \begin{cases}1 & w_{0} \leq w_{1} \\ 0 & \text { otherwise }\end{cases}
$$

As words are all orthogonal with respect to $\cdot Q_{+}^{-1} w_{1}$ contains $w_{0}$ with coefficient 1 whenever $w_{0} \leq w_{1}$, otherwise with coefficient 0 . Thus $Q_{+}^{-1} w$ is as claimed. The proof for $Q_{-}^{-1}$ is similar.

Now consider $Q_{-}$; the case of $Q_{+}$is similar. We will show that, for all $w, w_{0} \in \mathcal{M}_{n}^{e}$,

$$
\sum_{T \subseteq E_{w}^{x}}(-1)^{|T|}\left\langle\psi_{T}^{x} w \mid w_{0}\right\rangle=\left\langle\sum_{T \subseteq E_{w}^{x}}(-1)^{|T|} \psi_{T}^{x} w \mid w_{0}\right\rangle=w \cdot w_{0}= \begin{cases}1 & w_{0}=w, \\ 0 & \text { otherwise }\end{cases}
$$

which gives the desired expression for $Q_{-}$. Consider $f_{w}^{x}:\left\{1, \ldots, n_{x}\right\} \longrightarrow\left\{0, \ldots, n_{y}\right\}$, ie $f_{w}^{x}(i)$ is the number of $y$ 's to the left of the $i-\operatorname{th} x$ in $w$. Note that $\psi_{T}^{x}$ has the following effect on $f_{w}^{x}$ :

$$
f_{\psi_{T}^{x} w}^{x}(i)= \begin{cases}f_{w}^{x}(i) & \text { if the } x \text { numbered } i \text { is not in } T \\ f_{w}^{x}(i)+1 & \text { if the } x \text { numbered } i \text { is in } T .\end{cases}
$$


Thus, $\left\langle\psi_{T}^{x} w \mid w_{0}\right\rangle=1$ if and only if $f_{w}^{x}(i) \leq f_{w_{0}}^{x}(i)$ for all $i$ numbering $x$ 's of $w$ not in $T$, and $f_{w}^{x}(i)+1 \leq f_{w_{0}}^{x}(i)$ for all $i$ numbering $x$ 's of $w$ in $T$. Let $\left\{1, \ldots, n_{x}\right\}$ be partitioned into three sets

$$
\begin{aligned}
& S_{0}=\left\{i: f_{w}^{x}(i) \leq f_{w_{0}}^{x}(i)-1\right\}, \\
& S_{1}=\left\{i: f_{w}^{x}(i)=f_{w_{0}}^{x}(i)\right\}, \\
& S_{2}=\left\{i: f_{w}^{x}(i) \geq f_{w_{0}}^{x}(i)+1\right\} .
\end{aligned}
$$

We then have $\left\langle\psi_{T}^{x} w \mid w_{0}\right\rangle=1$ if and only if $T \subseteq S_{0}$ and $S_{2}=\varnothing$.

If $S_{2}$ is nonempty, then $\left\langle w \mid w_{0}\right\rangle=0$, and any $\left\langle\psi_{T}^{x} w \mid w_{0}\right\rangle=0$; also $w_{0} \cdot w=0$. Thus, we may assume $S_{2}=\varnothing$. Then $\left\langle\psi_{T}^{x} w \mid w_{0}\right\rangle=1$ if and only if $T \subseteq S_{0}$. Letting $T_{0}=S_{0} \cap E_{w}^{x}$, we then have

$$
\sum_{T \subseteq E_{w}^{x}}(-1)^{|T|}\left\langle\psi_{T}^{x} w \mid w_{0}\right\rangle=\sum_{T \subseteq T_{0}}(-1)^{|T|}\left\langle\psi_{T}^{x} w \mid w_{0}\right\rangle=\sum_{T \subseteq T_{0}}(-1)^{|T|}
$$

If $T_{0}$ is nonempty then this sum is 0 ; and also $S_{0}$ is nonempty, so $w_{0} \neq w$. If $T_{0}$ is empty then this sum is 1 and $E_{w}^{x} \subseteq S_{1}$, which means that $w \leq w_{0}$ and the exceptional $x$ 's in $w$ lie in the same positions as the corresponding $x$ in $w_{0}$; this implies $w=w_{0}$. So the expression for $Q_{-}$is as claimed.

\section{Corollary 2.8}

$$
H w=Q_{+} Q_{-}^{-1} w=\sum_{w_{i} \geq w} \sum_{T \subseteq E_{w_{i}}^{y}}(-1)^{|T|} \psi_{T}^{y} w_{i}
$$

We shall discuss $H$ in more detail in Sections 5.2-5.4. Note that all $Q_{ \pm}^{ \pm 1}$ and $H$ have integer coefficients. It follows that all of the isomorphisms are over $\mathbb{Z}$, not just over $\mathbb{Q}$.

\subsection{Differentials, commutation relations, normal form}

In quantum field theory, creation and annihilation operators $a^{*}, a$ usually satisfy $\left[a, a^{*}\right]=1$ or $\left\{a, a^{*}\right\}=1 ; a^{*}$ creates a particle, while $a$ annihilates each particle in turn and sums the results. Thus the compositions $a a^{*}$ and $a^{*} a$ count an identical set of situations, except that $a a^{*}$ counts the original state once more. Thus (for bosons) $a a^{*}-a^{*} a=1$ or (for fermions) $a a^{*}+a^{*} a=1$.

Our noncommutative setting is more symmetric: creations create once, and annihilations annihilate once. But if we take a sum (or alternating sum) over $i$ of annihilation 
operators $a_{s, i}$, we obtain the usual relations. Define operators

$$
a_{s}=\sum_{i=1}^{n_{s}} a_{s, i}, \quad d_{s}=\sum_{i=1}^{n_{s}}(-1)^{i} a_{s, i} \quad \text { on } \mathcal{F}_{n_{x}, n_{y}} .
$$

In the first case we obtain $\left[a_{s}, a_{s, 0}^{*}\right]=1$; in the second case we have $\left\{d_{s}, a_{s, 0}^{*}\right\}=1$.

Note that $a_{s}$ is simply partial differentiation by the symbol $s, a_{s}=\partial / \partial s$. It follows that $a_{x}, a_{y}$ commute and obey the Leibniz rule.

On the other hand, $d_{s}$ behaves like an exterior differential; $d_{s}^{2}=0 ; d_{s}\left(w_{0} w_{1}\right)=$ $\left(d_{s} w_{0}\right) w_{1}+(-1)^{k} w_{0}\left(d_{s} w_{1}\right)$ where $k$ is the degree of $w_{0}$ in $s$; and $d_{x} d_{y}=d_{y} d_{x}$. We may regard $d_{x}, d_{y}$ as boundary operators arising from the two simplicial structures on $\mathcal{F}$. (For this we could also take $d_{s}=\sum_{i=0}^{n_{s}+1}(-1)^{i} a_{s, i}$, the sum including terminal annihilations; this is also a differential, $d_{s}^{2}=0$ and $\left[d_{x}, d_{y}\right]=0$. Our choice fits better with the physical analogy.)

The differentials $d_{x}, d_{y}$ equip $\mathcal{F}$ with the structure of a double chain complex, and the relation $\left\{d_{s}, a_{s, 0}^{*}\right\}=1$ says that $a_{s, 0}^{*}$ is a chain homotopy from 1 to 0 ; thus the homology of this complex is trivial. In fact, the relation $d_{s} a_{s, 0}^{*}+a_{s, 0}^{*} d_{s}=1$ explicitly shows that any "closed" element $z, d_{s} z=0$, is a "boundary" since $d_{s} a_{s, 0}^{*} z+a_{s, 0}^{*} d_{s} z=$ $d_{s}\left(a_{s, 0}^{*} z\right)=z$. (cf [17, Proposition 7.5], following Frabetti [5].)

The simplicial set relations show us how to commute creation and annihilation operators of the same species $s \in\{x, y\}$. It follows that any sequence of $l s$-creation and $k$ $s$-annihilation operators can be written uniquely in the form

$$
a_{s, i_{1}}^{*} a_{s, i_{2}}^{*} \cdots a_{s, i_{k}}^{*} a_{s, j_{1}} a_{s, j_{2}} \cdots a_{s, j_{l}},
$$

where $i_{1}>i_{2}>\cdots>i_{k}$ (note strict inequality), and $j_{1} \geq j_{2} \geq \cdots \geq j_{l}$.

\subsection{Temperley-Lieb representation}

We now introduce more operators on $\mathcal{F}$ : for $s \in\{x, y\}$ and $0 \leq i \leq n_{s}$,

$$
T_{s, i}=a_{s, i}-a_{s, i+1}, \quad T_{s, i}^{*}=a_{s, i}^{*}-a_{s, i+1}^{*} .
$$

These $T_{s, i}$ have various obvious properties. For instance, with $1 \leq i \leq n_{s}-1, T_{s, i} w=0$ if and only if $w$ has the $i$-th and $(i+1)$-st $s$ symbols adjacent. Further,

$$
a_{s, i+1} T_{s, i}^{*}=a_{s, i+1}\left(a_{s, i}^{*}-a_{s, i+1}^{*}\right)=1-1=0,
$$

and so if we define

$$
U_{s, i}=T_{s, i}^{*} a_{s, i+1}=\left(a_{s, i}^{*}-a_{s, i+1}^{*}\right) a_{s, i+1}
$$


then $\left(U_{s, i}^{*}\right)^{2}=0$. For $1 \leq i \leq n_{s}-1$, the effect of $U_{s, i}$ on a word $w$ is to return 0 , if the $i$-th and $(i+2)-$ nd $s$ symbols are in the same block. Otherwise it gives difference between the two words, one obtained by moving the $(i+1)-$ st $s$ back to be adjacent to the $i$-th $s$, the other obtained by moving the $(i+1)-$ st $s$ forward to the $(i+2)-$ nd $s$. This makes combinatorially clear why $\left(U_{s, i}\right)^{2}=0$, and that $U_{s, i}$ and $U_{s, j}$ commute when $|i-j| \geq 2$.

Consider $U_{s, i}, U_{s, j}$ when $|i-j|=1$. We can compute (eg putting in normal form) that

$$
\begin{aligned}
U_{s, i} U_{s, i+1} U_{s, i} & =-U_{s, i}, \\
U_{s, i+1} U_{s, i} U_{s, i+1} & =-U_{s, i+1} .
\end{aligned}
$$

Thus the $U_{s, i}$ satisfy relations similar to the Temperley-Lieb algebra. This algebra is defined by the relations $U_{i}^{2}=\delta U_{i}, U_{i} U_{i+1} U_{i}=U_{i}, U_{i+1} U_{i} U_{i+1}=U_{i+1}$, and $U_{i} U_{j}=U_{j} U_{i}$ for $|i-j| \geq 2[20 ; 1 ; 2]$; we have $\delta=0$ and some sign changes, a "twisted" representation.

\subsection{A distinguished subset}

We will now define some distinguished elements in $\mathcal{F}$. Define

$$
\mathcal{C}^{1}=\left\{a_{s, i}^{*}, a_{s, i}, T_{s, i}^{*}\right\} \cdot 1 .
$$

That is, $\mathcal{C}^{1}$ is the orbit of 1 under the action of the operators $a_{s, i}^{*}, a_{s, i}, T_{s, i}^{*}$, over all $s \in\{x, y\}$ and all $0 \leq i \leq n_{s}+1$ in each $\mathcal{F}_{n}^{e}$. Similarly, define

$$
\begin{aligned}
& \mathcal{C}^{2}=\left\{a_{s, 0}^{*}, a_{s, 0}, H\right\} \cdot 1, \\
& \mathcal{C}^{3}=\left\{a_{s, n_{s}+1}^{*}, a_{s, n_{s}+1}, H\right\} \cdot 1 .
\end{aligned}
$$

In fact these are all the same (and one can easily find other sets of operators giving the same orbit).

Proposition 2.9 $\mathcal{C}^{1}=\mathcal{C}^{2}=\mathcal{C}^{3}$.

Denote this set $\mathcal{C}$. Let $\mathcal{C}_{n}=\mathcal{C} \cap \mathcal{F}_{n}, \mathcal{C}_{n_{x}, n_{y}}=\mathcal{C} \cap \mathcal{F}_{n_{x}, n_{y}}, \mathcal{C}_{n}^{e}=\mathcal{C} \cap \mathcal{F}_{n}^{e}$.

\section{Theorem 2.10}

(i) The set $\mathcal{C}$ is not closed under addition or multiplication by $\mathbb{Z}$. However $\mathcal{C}$ is closed under negation, multiplication, creation and annihilation operators, and the operators $T_{s, i}, T_{s, i}^{*}, U_{s, i}$. 
(ii) (a) Each element $v \in \mathcal{C}$ lies in some $\mathcal{F}_{n}^{e}\left(v \in \mathcal{C}_{n}^{e}\right)$ and is of the form $v=\sum_{i} a_{i} w_{i}$ where $a_{i}= \pm 1$ and the $w_{i}$ are distinct words in $\mathcal{M}_{n}^{e}$.

(b) Among the words $w_{i}$ there is a lexicographically first $w_{-}$and last $w_{+}$. Then for all $i, w_{-} \leq w_{i} \leq w_{+}$. If a word $w_{0}$ among the $w_{i}$ is comparable to all these $w_{i}$ with respect to $\leq$, then $w_{0} \in\left\{w_{-}, w_{+}\right\}$. If $w_{-} \neq w_{+}$then $\sum a_{i}=0$.

(c) For every pair $w_{-} \leq w_{+}$in $\mathcal{M}_{n}^{e}$ there are precisely two $v \in \mathcal{C}_{n}^{e}$ with $w_{-}, w_{+}$ being the lexicographically first and last words occurring in $v$; one is the negative of the other.

(d) The number of pairs $w_{-} \leq w_{+}$in $\mathcal{M}_{n}^{e}$ is the Narayana number $N_{n}^{e}$, and $\sum_{e} N_{n}^{e}=C_{n}$, the $n$-th Catalan number. Thus $\left|\mathcal{C}_{n}\right|=2 C_{n}$ and $\left|\mathcal{C}_{n}^{e}\right|=2 N_{n}^{e}$.

(iii) The operators $Q_{+}, Q_{-}$do not preserve $\mathcal{C}$; but $Q_{+} \mathcal{C}=Q_{-} \mathcal{C}$, and these have the same cardinality as $\mathcal{C}$ in each grading: $\left|Q_{ \pm} \mathcal{C}_{n}^{e}\right|=2 N_{n}^{e}$.

(iv) For any $v \in \mathcal{C},\langle v \mid v\rangle=1$ and $\langle v \mid H v\rangle=1$. For any $v_{0}, v_{1} \in \mathcal{C},\left\langle v_{0} \mid v_{1}\right\rangle \in$ $\{-1,0,1\}$.

(v) If the sum or difference of two distinct nonzero elements $u, v$ of $\mathcal{C}$ is also a nonzero element of $\mathcal{C}$, then after switching signs of $u, v$ and swapping $u, v$ if necessary, the triple is $u, v, u-v$, and $\langle u \mid v\rangle=1,\langle v \mid u\rangle=0$. There exists an operator $A^{*}: \mathcal{F}_{2}^{0} \longrightarrow \mathcal{F}_{n}^{e}$, which is a composition of initial creation operators and applications of $H$, such that $A(x y)=u$ and $A(y x)=v$.

(vi) If $u, v \in \mathcal{C}$ and $\langle u \mid v\rangle=1$, then there exists a sequence $u=v_{0}, v_{1}, \ldots, v_{m}=v$ in $\mathcal{C}$ such that each $v_{i}-v_{i+1} \in \mathcal{C}$; and for each $i \leq j,\left\langle v_{i} \mid v_{j}\right\rangle=1$.

The proof of this theorem will be given via isomorphism with sutured TQFT in Section 5.1. The Narayana numbers $N_{n}^{e}$ here are as defined in [17].

There are some further tenuous physical analogies and speculations. Elements of $\mathcal{C}$ can be regarded as "pure states". The operator $H$ can perhaps be considered as a Hamiltonian generating a time evolution, its periodicity some manifestation of a term $e^{i H t}$. The operators $Q_{ \pm}$, which taken together define $H$, can be considered as supersymmetry; $Q_{+} \mathcal{C}$ can be regarded as super-partner-states. Is there some interpretation of sutured manifolds with corners having spacelike and timelike boundaries, and a Hamiltonian giving something like a partition function for contact manifolds?

\section{Sutured TQFT}

\subsection{Sutured surfaces}

For our purposes, a sutured 3-manifold $(M, \Gamma)$ is a 3-manifold with boundary $M$, with $\Gamma \subset \partial M$ an embedded oriented 1-manifold, such that $\partial M \backslash \Gamma=R_{+} \cup R_{-}$, where 
$\partial R_{ \pm}=\Gamma$ as oriented $1-$ manifolds, and $R_{ \pm}$is oriented as $\pm \partial M$. (Crossing $\Gamma$ along $\partial M$ goes from $R_{ \pm}$to $R_{\mp}$.)

A sutured 3-manifold may have corners on its boundary; there may be a curve $C$ on $\partial M$ along which two smooth surfaces meet. If so, sutures are required not to match along $C$, but to interleave; see Figure 2. The surface may be smoothed; in doing so, sutures are rounded and complementary regions $R_{ \pm}$joined as shown.
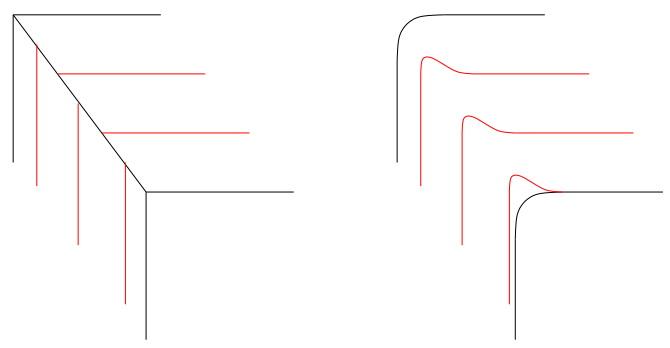

Figure 2: Edge smoothing and unsmoothing

In the following, we take sutures on surfaces, but without any associated 3-manifold. Define a sutured surface $(\Sigma, \Gamma)$ to be a compact oriented surface $\Sigma$, possibly disconnected, with $\Gamma \subset \Sigma$ a properly embedded oriented 1-submanifold; $\Gamma$ must have the property that $\Sigma \backslash \Gamma=R_{+} \cup R_{-}$, where $R_{ \pm}$is oriented as $\pm \Sigma$, and $\overline{\partial R_{ \pm} \backslash \partial \Sigma}=\Gamma$ as oriented 1-manifolds. Again $(\Sigma, \Gamma)$ may have corners, with interleaving sutures, which may be smoothed as above. In this paper, we will only consider sutured surfaces with $\partial \Sigma \neq \varnothing$.

We define also a sutured background surface $(\Sigma, F)$, which is a compact oriented surface $\Sigma$ (possibly disconnected) with $\partial \Sigma \neq \varnothing$, together with a finite set of signed points $F \subset \partial \Sigma$, such that $\partial \Sigma \backslash F=C_{+} \cup C_{-}$, where $C_{ \pm}$are oriented as $\pm \partial \Sigma$, and $\partial C_{ \pm}=F$ as signed points. (Hence each boundary component $C$ of $\Sigma$ has a positive even number of points of $F$, which cut it alternately into arcs of $C_{+}$and $C_{-}$.) Again a sutured background surface may have corners. A set of sutures $\Gamma$ on a sutured background surface $(\Sigma, F)$ is an oriented properly embedded 1-submanifold of $\Sigma$ such that $\partial \Gamma=\partial \Sigma \cap \Gamma=F$ and such that $(\Sigma, \Gamma)$ is a sutured surface, with $\partial R_{ \pm}=\Gamma \cup C_{ \pm}$. Given a sutured background surface $(\Sigma, F)$ with $\partial \Sigma \backslash F=C_{+} \cup C_{-}$as above, we define gluings of it. Consider two disjoint 1 -manifolds $G_{0}, G_{1} \subseteq \partial \Sigma$, and a homeomorphism $\tau: G_{0} \cong G_{1}$ which identifies marked points and positive/negative arcs, $G_{0} \cap F \cong$ $G_{1} \cap F, G_{0} \cap C_{ \pm} \cong G_{1} \cap C_{ \pm}$. Then gluing $(\Sigma, F)$ along $\tau$ gives a surface $\#_{\tau}(\Sigma, F)$. If there remain marked points on each boundary component then $\#_{\tau}(\Sigma, F)$ is also a sutured background surface and we call $\tau$ a sutured gluing map. If $\Gamma$ is a set of sutures on $(\Sigma, F)$ then a sutured gluing map gives a glued set of sutures $\#_{\tau} \Gamma$ on $\#_{\tau}(\Sigma, F)$. 
A set of sutures $\Gamma$ on $(\Sigma, F)$ has a (relative) Euler class $e(\Gamma)=\chi\left(R_{+}\right)-\chi\left(R_{-}\right)$.

On the disc sutured background $\left(D^{2}, F_{n+1}\right)$ with $\left|F_{n+1}\right|=2(n+1)$, drawn in the plane and inheriting its orientation, we label the points of $F_{n+1}$ with integers mod $2(n+1)$ as in [17]. We choose a basepoint, numbered 0 ; the arc of $\partial D^{2}$ immediately clockwise (resp. anticlockwise) of 0 must be signed positive (resp. negative). The points of $F_{n+1}$ are numbered clockwise, modulo $2(n+1)$.

A set of sutures $\Gamma$ on $\left(D^{2}, F_{n+1}\right)$ without contractible components is just a chord diagram; it clearly has $|e(\Gamma)| \leq n$ and $e(\Gamma) \equiv n \bmod 2$. Given a chord diagram $\Gamma$ on $\left(D^{2}, F_{n+1}\right)$, from $n$ and $e$ we define $n_{-}=(n-e) / 2, n_{+}=(n+e) / 2$, so $n_{-}, n_{+}$ are nonnegative integers, $n=n_{-}+n_{+}$and $e=n_{+}-n_{-}$. The point of $F$ numbered $2 n_{+}+1 \equiv-2 n_{-}-1 \bmod 2(n+1)$ is called the root point. Cutting $\partial D^{2}$ at base and root points gives two arcs. The arc containing $1, \ldots, 2 n_{+}$is called the eastside. The arc containing $-1, \ldots,-2 n_{-}$is called the westside.

\subsection{TQFT axioms}

As in any TQFT, we associate algebraic objects to our topological objects. Here the topological objects are sutured background surfaces, sets of sutures, and gluings. A sutured TQFT is a set of assignments as described by the following axioms.

Axiom 1 To each sutured background surface $(\Sigma, F)$, assign an abelian group $V(\Sigma, F)$, depending only on the homeomorphism type of the pair $(\Sigma, F)$.

Axiom 2 To a set of sutures $\Gamma$ on $(\Sigma, F)$, assign a subset of suture elements $c(\Gamma) \subset$ $V(\Sigma, F)$, depending only on the isotopy class of $\Gamma$ relative to boundary.

(We would have liked a single suture element, but this will turn out not to be possible, as we discuss in Section 3.4. Quantum states differing by a unit are physically indistinguishable.)

Axiom 3 For a sutured gluing map $\tau$ of a sutured background surface $(\Sigma, F)$, assign a collection of linear maps $\Phi_{\tau}^{i}: V(\Sigma, F) \longrightarrow V\left(\#_{\tau}(\Sigma, F)\right)$.

(We would have liked one canonical map, but this will turn out not to be possible.)

Axiom 4 For a finite disjoint union of sutured background surfaces $\bigsqcup_{i}\left(\Sigma_{i}, F_{i}\right)$,

$$
V\left(\bigsqcup_{i}\left(\Sigma_{i}, F_{i}\right)\right)=\bigotimes_{i} V\left(\Sigma_{i}, F_{i}\right) .
$$


Axiom 5 If $\Gamma$ is a set of sutures on $(\Sigma, F)$ and $\tau$ is a gluing of $(\Sigma, F)$ then each $\Phi_{\tau}^{i}$ takes suture elements to suture elements surjectively, $c(\Gamma) \rightarrow c\left(\#_{\tau} \Gamma\right)$.

These conditions are not standard TQFT axioms but are in a similar spirit.

It follows from the above that any $V(\Sigma, F)$ can be interpreted as a space of operators. Take some components of $\partial \Sigma$ and call them incoming; call the rest outgoing. Write $\partial \Sigma=(\partial \Sigma)_{\text {in }} \cup(\partial \Sigma)_{\text {out }}$ and $F=F_{\text {in }} \cup F_{\text {out }}$. Suppose we have a sutured background surface $\left(\Sigma_{\text {in }}, F_{\text {in }}\right)$ which has boundary $\left(\partial \Sigma_{\text {in }}\right)$ identified with $(\partial \Sigma)_{\text {in }}$ by a gluing $\tau$; and $\tau$ identifies $F_{\text {in }}$ and positive/negative boundary arcs on both surfaces. The gluing gives a sutured background surface $\left(\Sigma_{\text {out }}, F_{\text {out }}\right)$, where $\Sigma_{\text {out }}=\Sigma_{\text {in }} \cup \Sigma$, and a (possibly not unique) map

$$
\Phi_{\tau}: V\left(\Sigma_{\text {in }}, F_{\text {in }}\right) \otimes V(\Sigma, F) \longrightarrow V\left(\Sigma_{\text {out }}, F_{\text {out }}\right),
$$

which is natural with respect to suture elements: if $\Gamma, \Gamma_{\text {in }}$ are respectively sets of sutures on $(\Sigma, F)$ and $\left(\Sigma_{\text {in }}, F_{\text {in }}\right)$, then $\Gamma_{\text {out }}=\Gamma_{\text {in }} \cup \Gamma$ is a set of sutures on $\left(\Sigma_{\text {out }}, F_{\text {out }}\right)$, and $\Phi_{\tau}$ maps $c\left(\Gamma_{\text {in }}\right) \otimes c(\Gamma) \rightarrow c\left(\Gamma_{\text {out }}\right)$.

Thus, a choice of sutures $\Gamma$ on $(\Sigma, F)$, together with a choice of representative $c \in$ $c(\Gamma) \subset V(\Sigma, F)$ gives a specific $V\left(\Sigma_{\text {in }}, F_{\text {in }}\right) \longrightarrow V\left(\Sigma_{\text {out }}, F_{\text {out }}\right)$ which we denote $\Phi_{\tau, c}$ :

$$
\Phi_{\tau, c}: V\left(\Sigma_{\text {in }}, F_{\text {in }}\right) \longrightarrow V\left(\Sigma_{\text {out }}, F_{\text {out }}\right), \quad x \mapsto \Phi_{\tau}(x \otimes c) .
$$

This is perhaps closer in spirit to a cobordism map. In this formulation, we view the situation as an inclusion of background surfaces $\left(\Sigma_{\text {in }}, F_{\text {in }}\right) \hookrightarrow\left(\Sigma_{\text {out }}, F_{\text {out }}\right)$, together with a set of sutures $\Gamma$ on $\left(\Sigma_{\text {out }} \backslash \Sigma_{\text {in }}, F_{\text {in }} \cup F_{\text {out }}\right)$. Note that the inclusion must be strict in the sense that $\Sigma_{\text {in }}$ lies in the interior of $\Sigma_{\text {out }}$. In this way we can regard our "TQFT" is a " $2+1=2$-dimensional TQFT".

In fact, we can reformulate gluing Axioms 3 and 5 in terms of inclusions, in line with [10].

Axiom $3^{\prime}$ To an inclusion $\iota\left(\Sigma_{\text {in }}, F_{\text {in }}\right) \hookrightarrow\left(\Sigma_{\text {out }}, F_{\text {out }}\right)$ of sutured background surfaces, with $\Sigma_{\text {in }}$ lying in the interior of $\Sigma_{\text {out }}$, together with $\Gamma$ a set of sutures on $\left(\Sigma_{\text {out }} \backslash \Sigma_{\text {in }}, F_{\text {in }} \cup F_{\text {out }}\right)$, assign a collection of linear maps $\Phi_{\iota, \Gamma}^{i}: V\left(\Sigma_{\text {in }}, F_{\text {in }}\right) \longrightarrow$ $V\left(\Sigma_{\text {out }}, F_{\text {out }}\right)$.

Axiom 5' If $\Gamma_{\text {in }}$ is a set of sutures on $\left(\Sigma_{\text {in }}, F_{\text {in }}\right)$, let $\Gamma_{\text {out }}=\Gamma_{\text {in }} \cup \Gamma$ be the corresponding set of sutures on $\left(\Sigma_{\text {out }}, F_{\text {out }}\right)$. Then each $\Phi_{\iota, \Gamma}^{i}$ maps takes suture elements to suture elements surjectively, $c\left(\Gamma_{\text {in }}\right) \rightarrow c\left(\Gamma_{\text {out }}\right)$.

Lemma 3.1 Axioms 3 and 5 are equivalent to Axioms $3^{\prime}$ and $5^{\prime}$. 
Proof Assuming Axioms 3 and 5, the above discussion shows how to regard the inclusion $\iota:\left(\Sigma_{\text {in }}, F_{\text {in }}\right) \hookrightarrow\left(\Sigma_{\text {out }}, F_{\text {out }}\right)$ as a gluing $\tau$; for each choice of suture element $c \in c(\Gamma)$, there are maps $V\left(\Sigma_{\text {in }}, F_{\text {in }}\right) \longrightarrow V\left(\Sigma_{\text {out }}, F_{\text {out }}\right)$. We take all the maps so obtained over the possible choices for $c$.

In the other direction, assume Axioms $3^{\prime}$ and $5^{\prime}$. Given a gluing $\tau$ of $(\Sigma, F)$, removing a neighbourhood of the boundary of $(\Sigma, F)$, after gluing, gives an inclusion $\iota:(\Sigma, F) \hookrightarrow \#_{\tau}(\Sigma, F)$, and lying in its interior. Moreover, there is a natural set of sutures $\Gamma$ on a neighbourhood of the boundary of $(\Sigma, F)$, taking a product neighbourhood of the boundary. We obtain inclusion maps $\Phi_{\iota, \Gamma}^{i}: V(\Sigma, F) \longrightarrow V\left(\#_{\tau}(\Sigma, F)\right)$, which we define to be $\Phi_{\tau}^{i}$. (See [10, Figure 13].)

We now impose the condition that "overtwisted contact elements are zero".

Axiom 6 If $\Gamma$ contains a closed contractible loop then $c(\Gamma)=\{0\}$.

(Perhaps more generally we could set $c(\Gamma)$ to be some power of an indeterminate $\delta$; this would be closer to analogous situations in knot theory. But the analogy from contact geometry suggests that one closed loop is equivalent to many.)

Consider $\left(D^{2}, F_{1}\right)$, the disc with two marked points. There is only one chord diagram on $\left(D^{2}, F_{1}\right)$. We call this $\Gamma$ the vacuum $\Gamma_{\varnothing}$, and $\left(D^{2}, F_{1}\right)$ the vacuum background. We will impose the standard QFT condition that the vacuum is nonzero, and normalise.

Axiom $7 \quad V\left(D^{2}, F_{1}\right)=\mathbb{Z}$ and $c\left(\Gamma_{\varnothing}\right) \subseteq\{-1,1\}$.

In more generality, we could set $V\left(D^{2}, F_{1}\right)$ to be a ground ring $R$ and then say that sutured TQFT is over $R$ coefficients. In [17] we essentially considered sutured TQFT over $\mathbb{Z}_{2}$; here we focus on $\mathbb{Z}$ coefficients, though we will need to make reference to $\mathbb{Z}_{2}$ coefficients and previous work at times.

For any sutured background $(\Sigma, F)$ with sutures $\Gamma$, there is an inclusion $\left(D^{2}, F_{1}\right) \hookrightarrow$ $(\Sigma, F)$, and sutures $\Gamma^{\prime}$ on $\left(\Sigma \backslash D^{2}, F \cup F_{1}\right)$, taking $\Gamma_{\varnothing} \mapsto \Gamma_{\varnothing} \cup \Gamma^{\prime}=\Gamma$. Axiom $3^{\prime}$ gives a map $\mathbb{Z} \cong V\left(D^{2}, F_{1}\right) \longrightarrow V(\Sigma, F)$ taking $c\left(\Gamma_{\varnothing}\right) \subseteq\{-1,1\} \rightarrow c(\Gamma)$ surjectively and we immediately have the following.

Lemma 3.2 For any sutures $\Gamma$ on any $(\Sigma, F), c(\Gamma)$ is either a singleton or is of the form $\{c,-c\}$.

Over $\mathbb{Z}_{2}$ coefficients, these two possibilities are the same, and $c(\Gamma)$ is always a single element. 
Any disc with a chord diagram may be included into a larger sutured disc in which the sutures simplify to the vacuum. The corresponding inclusion gives a map $V\left(D^{2}, F_{n}\right) \longrightarrow$ $V\left(D^{2}, F_{1}\right)=\mathbb{Z}$ which takes $c(\Gamma) \rightarrow c\left(\Gamma_{\varnothing}\right) \subseteq\{-1,1\}$ surjectively.

Lemma 3.3 If $\Gamma$ is a chord diagram then every $c \in c(\Gamma)$ is nonzero, primitive and nontorsion.

The "interesting" elements in each $V(\Sigma, F)$ are the suture elements. Largely for reasons of convenience, we will require our sutured TQFT to be "minimal" in the following sense.

Axiom 8 Every $V(\Sigma, F)$ is spanned by suture elements.

A "free presutured TQFT" is easily defined satisfying Axioms 1-8; it will not satisfy Axiom 9, but we shall consider it in Section 3.13. Take $V(\Sigma, F)=\bigoplus_{\Gamma} \mathbb{Z}_{\Gamma}$, freely generated by elements $c_{\Gamma}$, one for each isotopy class of sutures $\Gamma$ on $(\Sigma, F)$ without contractible loops. We set $c(\Gamma)=\left\{c_{\Gamma}\right\}$ if $\Gamma$ has no contractible loops, otherwise $c(\Gamma)=\{0\}$. Gluing maps are defined in the obvious way, $\Phi_{\tau} c_{\Gamma}=c_{\#_{\tau} \Gamma}$ or 0 accordingly as contractible components are seen or not. Clearly Axioms 1-8 are satisfied.

The final axiom, which essentially gives all the structure in the TQFT, involves the "stacking" bilinear form $\langle\cdot \mid \cdot\rangle: V\left(D^{2}, F_{n}\right) \otimes V\left(D^{2}, F_{n}\right) \longrightarrow \mathbb{Z}$. Consider the cylinder $S^{1} \times[0,1]$, with $2 n$ parallel sutures $\{\cdot\} \times[0,1]$; and then remove a small neighbourhood of a point on one of those sutures (a "leak"). Call this sutured surface $(\Sigma, \Gamma)$; topologically $\Sigma$ is a pair of pants. Regard the two ends of the cylinder as incoming and the "leak" as outgoing. Consider gluing two incoming sutured background discs $\left(D^{2}, F_{n}\right)$ to $(\Sigma, \Gamma)$; we glue them to the ends of the cylinder along corners as in $\partial\left(D^{2} \times[0,1]\right)$; after gluing and rounding, we have the vacuum background $\left(D^{2}, F_{1}\right)$. Choosing a $c \in c(\Gamma) \subset V(\Sigma, \Gamma)$, we obtain a map

$$
\langle\cdot \mid \cdot\rangle=\Phi_{\tau, c}: V\left(D^{2}, F_{n}\right) \otimes V\left(D^{2}, F_{n}\right) \longrightarrow V\left(D^{2}, F_{1}\right)=\mathbb{Z} .
$$

(There may be several choices for $\langle\cdot \mid \cdot\rangle$. For now, make an arbitrary choice; we may adjust it later.) Thus $\langle\cdot \mid \cdot\rangle$ describes "inclusion into a leaky cylinder". If we have sutures $\Gamma_{0}, \Gamma_{1}$ on $\left(D^{2}, F_{n}\right)$ and $c_{0} \in c\left(\Gamma_{0}\right), c_{1} \in c\left(\Gamma_{1}\right)$, then gluing them into the leaky cylinder at $S^{1} \times\{0\}, S^{1} \times\{1\}$ respectively, and rounding corners, gives a set of sutures on $\left(D^{2}, F_{1}\right)$. Either this is the vacuum, and $\left\langle c_{0} \mid c_{1}\right\rangle= \pm 1$, or there is a contractible suture, and $\left\langle c_{0} \mid c_{1}\right\rangle=0$. Forgetting the leak, $\left\langle c_{0} \mid c_{1}\right\rangle= \pm 1$ (resp. 0) if and only if after rounding corners on the cylinder, we have a sutured sphere with connected (resp. disconnected) sutures. 
Axiom 9 Suppose two elements $x, y \in V\left(D^{2}, F_{n}\right)$ have the following property: for any set of sutures $\Gamma$ on $\left(D^{2}, F_{n}\right)$, there exists $c \in c(\Gamma)$ such that $\langle x \mid c\rangle= \pm\langle y \mid c\rangle$. Then $x= \pm y$.

As mentioned in the introduction, this axiom is essentially equivalent to the bypass relation. The formulation of nondegeneracy is somewhat unorthodox; in Section 3.3 we consider various formulations and their equivalence or nonequivalence.

Note that Axiom 9 is limited to discs; stacking more complicated surfaces does not map into the vacuum background. We can now give a precise version of Theorem 1.1. A much more general statement is given in Theorem 5.1.

Theorem 3.4 In any sutured TQFT, there is an isomorphism

$$
\left(V\left(D^{2}, F_{n+1}\right),\langle\cdot \mid \cdot\rangle\right) \cong\left(\mathcal{F}_{n},\langle\cdot \mid \cdot\rangle\right) \text {. }
$$

\subsection{Nondegeneracy axioms and bypass relations}

Axiom 9 implies certain relations between suture elements. Consider $\left(D^{2}, F_{3}\right)$, and the three sets of sutures $\Gamma_{0}, \Gamma_{1}, \Gamma_{2}$ shown in Figure 3. Let $c_{i} \in c\left(\Gamma_{i}\right) \subset V\left(D^{2}, F_{3}\right)$ be suture elements. We easily obtain
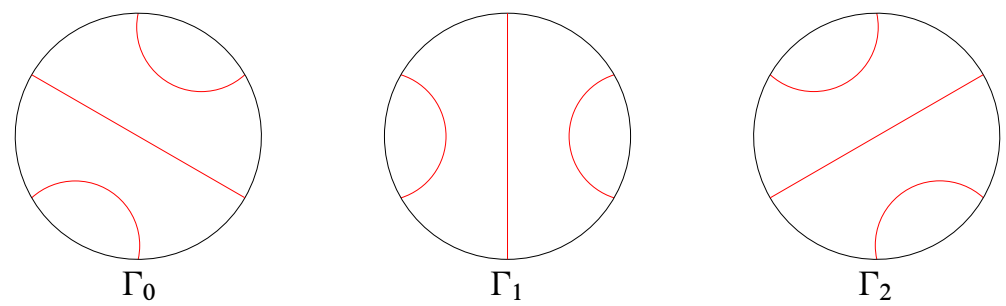

Figure 3: Sutures in the bypass relation

$$
\begin{array}{lll}
\left\langle c_{0} \mid c_{0}\right\rangle= \pm 1, & \left\langle c_{0} \mid c_{1}\right\rangle= \pm 1, & \left\langle c_{0} \mid c_{2}\right\rangle=0 \\
\left\langle c_{1} \mid c_{0}\right\rangle=0, & \left\langle c_{1} \mid c_{1}\right\rangle= \pm 1, & \left\langle c_{1} \mid c_{2}\right\rangle= \pm 1 \\
\left\langle c_{2} \mid c_{0}\right\rangle= \pm 1, & \left\langle c_{2} \mid c_{1}\right\rangle=0, & \left\langle c_{2} \mid c_{2}\right\rangle= \pm 1 .
\end{array}
$$

Take $\alpha=c_{0} \pm c_{1}$, with the plus or minus chosen so that $\left\langle\alpha \mid c_{1}\right\rangle=0$. Then we have

$$
\left\langle\alpha \mid c_{0}\right\rangle= \pm 1, \quad\left\langle\alpha \mid c_{1}\right\rangle=0, \quad\left\langle\alpha \mid c_{2}\right\rangle= \pm 1
$$

Thus, by Axiom 9, $\alpha= \pm c_{2}$; hence $c_{2}= \pm c_{0} \pm c_{1}$ for some choice of sign.

On a sutured surface $(\Sigma, \Gamma)$, we may consider the operation of removing an embedded disc $D$ in the interior of $\Sigma$ on which the sutures are isotopic (rel boundary of the smaller 
disc) to a set shown in Figure 3 above; and then replacing the sutures on this smaller disc with a different set shown in Figure 3. Such an operation is called bypass surgery and comes in two versions: $u p$, which takes $\Gamma_{0} \mapsto \Gamma_{1} \mapsto \Gamma_{2} \mapsto \Gamma_{0}$; and down, which takes $\Gamma_{0} \mapsto \Gamma_{2} \mapsto \Gamma_{1} \mapsto \Gamma_{0}$. Bypass surgery preserves Euler class; bypass-related sutured surfaces naturally come in triples. If $\Gamma_{0}^{\prime}, \Gamma_{1}^{\prime}, \Gamma_{2}^{\prime}$ are a bypass-related triple of sutures on $(\Sigma, F)$, consider including the bypass surgery disc into $\Sigma$ (with fixed sutures); we obtain a map $V\left(D^{2}, F_{3}\right) \longrightarrow V(\Sigma, F)$ taking each $c\left(\Gamma_{i}\right) \rightarrow c\left(\Gamma_{i}^{\prime}\right)$; thus the linear dependency persists.

Lemma 3.5 Let $\Gamma_{0}, \Gamma_{1}, \Gamma_{2}$ be a bypass related triple of sets of sutures on a sutured background $(\Sigma, F)$. Choose any $c_{i} \in c\left(\Gamma_{i}\right)$. Then there exists a choice of signs such that $c_{0}= \pm c_{1} \pm c_{2}$.

In sutured TQFT over $\mathbb{Z}_{2}$, of course $c_{0}, c_{1}, c_{2}$ are single elements and $c_{0}+c_{1}+c_{2}=0$. In [17] we defined groups $S F H_{\text {comb }}(T, n)$, which were generated by chord diagrams of $n$ chords, subject to the relation that bypass related triples sum to zero.

Proposition 3.6 In any sutured TQFT over $\mathbb{Z}_{2}$,

$$
V\left(D^{2}, F_{n+1}\right) \cong S F H_{\mathrm{comb}}(T, n+1) \cong \mathbb{Z}_{2}^{2^{n}} .
$$

This isomorphism takes a chord diagram $\Gamma$ in $\operatorname{SFH}_{\mathrm{comb}}(T, n+1)$ to the suture element $c(\Gamma) \in V\left(D^{2}, F_{n+1}\right)$.

The proof is almost immediate from the definition of sutured TQFT, given our previous work; it is given in Section 3.6.

As the formulation of nondegeneracy in Axiom 9 is a little unorthodox, we can consider whether other formulations are equivalent; and the equivalence to various formulations of the bypass relation.

Proposition 3.7 In the presence of Axioms 1-8 of sutured TQFT, the following axioms are equivalent.

(i) Suppose two elements $x, y \in V\left(D^{2}, F_{n}\right)$ have the following property: for any set of sutures $\Gamma$ on $\left(D^{2}, F_{n}\right)$ and for all $c \in c(\Gamma),\langle x \mid c\rangle= \pm\langle y \mid c\rangle$. Then $x= \pm y$.

(ii) (Original Axiom 9) Suppose two elements $x, y \in V\left(D^{2}, F_{n}\right)$ have the following property: for any set of sutures $\Gamma$ on $\left(D^{2}, F_{n}\right)$, there exists $c \in c(\Gamma)$ such that $\langle x \mid c\rangle= \pm\langle y \mid c\rangle$. Then $x= \pm y$.

(iii) Suppose two elements $x, y \in V\left(D^{2}, F_{3}\right)$ have the following property: for any set of sutures $\Gamma$ on $\left(D^{2}, F_{3}\right)$ and for all $c \in c(\Gamma),\langle x \mid c\rangle= \pm\langle y \mid c\rangle$. Then $x= \pm y$. 
(iv) Suppose two elements $x, y \in V\left(D^{2}, F_{3}\right)$ have the following property: for any set of sutures $\Gamma$ on $\left(D^{2}, F_{3}\right)$, there exists $c \in c(\Gamma)$ such that $\langle x \mid c\rangle= \pm\langle y \mid c\rangle$. Then $x= \pm y$.

(v) Suppose $\Gamma_{0}, \Gamma_{1}, \Gamma_{2}$ are a bypass triple of sutures on $\left(D^{2}, F_{n}\right)$. For any $c_{0}, c_{1}, c_{2}$ suture elements in $c\left(\Gamma_{0}\right), c\left(\Gamma_{1}\right), c\left(\Gamma_{2}\right)$ respectively, there exist $\epsilon_{1}, \epsilon_{2} \in\{-1,1\}$ such that $c_{0}=\epsilon_{1} c_{1}+\epsilon_{2} c_{2}$.

(vi) Suppose $\Gamma_{0}, \Gamma_{1}, \Gamma_{2}$ are a bypass triple of sutures on $\left(D^{2}, F_{n}\right)$. Then there exist $c_{0}, c_{1}, c_{2}$ suture elements in $c\left(\Gamma_{0}\right), c\left(\Gamma_{1}\right), c\left(\Gamma_{2}\right)$ respectively, and $\epsilon_{1}, \epsilon_{2} \in$ $\{-1,1\}$ such that $c_{0}=\epsilon_{1} c_{1}+\epsilon_{2} c_{2}$.

(vii) Suppose $\Gamma_{0}, \Gamma_{1}, \Gamma_{2}$ are a bypass triple of sutures on $\left(D^{2}, F_{3}\right)$. For any $c_{0}, c_{1}, c_{2}$ suture elements in $c\left(\Gamma_{0}\right), c\left(\Gamma_{1}\right), c\left(\Gamma_{2}\right)$ respectively, there exist $\epsilon_{1}, \epsilon_{2} \in\{-1,1\}$ such that $c_{0}=\epsilon_{1} c_{1}+\epsilon_{2} c_{2}$.

(viii) Suppose $\Gamma_{0}, \Gamma_{1}, \Gamma_{2}$ are a bypass triple of sutures on $\left(D^{2}, F_{3}\right)$. Then there exist $c_{0}, c_{1}, c_{2}$ suture elements in $c\left(\Gamma_{0}\right), c\left(\Gamma_{1}\right), c\left(\Gamma_{2}\right)$ respectively, and $\epsilon_{1}, \epsilon_{2} \in$ $\{-1,1\}$ such that $c_{0}=\epsilon_{1} c_{1}+\epsilon_{2} c_{2}$.

Given the foregoing, it's not difficult to see that (i) $\Leftrightarrow$ (ii) $\Rightarrow$ (iii) $\Leftrightarrow$ (iv) $\Rightarrow$ (v) $\Leftrightarrow$ (vi) $\Leftrightarrow($ vii $) \Leftrightarrow$ (viii). The difficult part is to show ((v)-(viii)) $\Rightarrow((\mathrm{i})-(\mathrm{ii}))$; we do this in Section 3.13.

A more orthodox form of nondegeneracy in fact is not equivalent.

Proposition 3.8 In the presence of Axioms 1-8, the following two axioms are equivalent.

(i) Suppose $x \in V\left(D^{2}, F_{n}\right)$ has the following property: for any set of sutures $\Gamma$ on $\left(D^{2}, F_{n}\right)$ and for any suture element $c \in c(\Gamma),\langle x \mid c\rangle=0$. Then $x=0$.

(ii) Suppose $x \in V\left(D^{2}, F_{n}\right)$ has the following property: for any $y \in V\left(D^{2}, F_{n}\right)$, $\langle x \mid y\rangle=0$. Then $x=0$.

There exists a sutured TQFT obeying Axioms 1-8 and these two alternative Axioms 9, such that $V\left(D^{2}, F_{n}\right) \cong \mathbb{Z}^{C_{n}}$. Here $C_{n}$ is the $n-t h$ Catalan number; denoting the $C_{n}$ chord diagrams on $\left(D^{2}, F_{n}\right)$ by $\left\{\Gamma_{i}\right\}_{i=1}^{C_{n}}$, each $c\left(\Gamma_{i}\right)=\left\{ \pm c_{i}\right\}$ and the $c_{i}$ form a basis for each $V\left(D^{2}, F_{n}\right)$.

The proof is given in Section 3.13. We will construct the example explicitly; there will be no linear relation between suture elements for distinct chord diagrams, let alone a bypass relation. 


\subsection{Impossibility of coherent signs}

It is possible to have each $c(\Gamma)$ a single element over $\mathbb{Z}_{2}$. But if $c\left(\Gamma_{\varnothing}\right)$ is nontorsion it is impossible, and it remains impossible even relaxing several axioms. This is a pedantic version of material in [10].

\section{Proposition 3.9 Consider making assignments:}

$\left(1^{\prime}\right)$ to each (homeomorphism class of) $\left(D^{2}, F_{n}\right)$, an abelian group $V\left(D^{2}, F_{n}\right)$;

$\left(2^{\prime}\right)$ to each (isotopy class of) set of sutures $\Gamma$ on $\left(D^{2}, F_{n}\right)$, a suture element $c(\Gamma) \in$ $V\left(D^{2}, F_{n}\right)$;

(3') to an inclusion $\left(D^{2}, F_{n}\right) \hookrightarrow\left(D^{2}, F_{m}\right)$ with a set of sutures $\Gamma$ on the intermediate $\left(S^{1} \times I, F_{n} \cup F_{m}\right)$, a collection of linear maps $\Phi^{i}: V\left(D^{2}, F_{n}\right) \longrightarrow V\left(D^{2}, F_{m}\right)$.

Suppose such assignments satisfy:

$\left(5^{\prime}\right)$ Each $\Phi^{i}$ is natural with respect to suture elements, ie for a set of sutures $\Gamma^{\prime}$ on $\left(D^{2}, F_{n}\right), c\left(\Gamma^{\prime}\right) \mapsto c\left(\Gamma^{\prime} \cup \Gamma\right)$.

(6) If $\Gamma$ contains a closed contractible loop then $c(\Gamma)=0$.

$\left(7^{\prime}\right) \quad c\left(\Gamma_{\varnothing}\right)$ is torsion-free.

$\left(9^{\prime}\right)$ Let $\Gamma_{0}, \Gamma_{1}, \Gamma_{2}$ be the three sets of sutures on $\left(D^{2}, F_{3}\right)$ in Figure 3. Then the three suture elements $c\left(\Gamma_{0}\right), c\left(\Gamma_{1}\right), c\left(\Gamma_{2}\right) \in V\left(D^{2}, F_{3}\right)$ are linearly dependent over $\mathbb{Z}$.

Then all suture elements are 0 .

Here primed axioms are weaker versions of the original; Axioms 4 and 8 are omitted altogether.

Proof Consider the chord diagrams $\Gamma_{0}, \Gamma_{1}, \Gamma_{2}$ on $\left(D^{2}, F_{3}\right)$ in Figure 3 ; and including them into $\left(D^{2}, F_{2}\right)$ using the sets of sutures $\Gamma_{a}, \Gamma_{b}, \Gamma_{c}$ on the intermediate annulus in Figure 4. From Axiom $3^{\prime}$ then we obtain maps $\Phi_{a}, \Phi_{b}, \Phi_{c}: V\left(D^{2}, F_{3}\right) \longrightarrow$ $V\left(D^{2}, F_{2}\right)$.

Letting $\Gamma_{-}, \Gamma_{+}$be as shown in Figure 5, we immediately obtain

$$
\begin{array}{lll}
\Phi_{a}: c\left(\Gamma_{0}\right) \mapsto c\left(\Gamma_{+}\right) & c\left(\Gamma_{1}\right) \mapsto c\left(\Gamma_{+}\right) & c\left(\Gamma_{2}\right) \mapsto 0, \\
\Phi_{b}: c\left(\Gamma_{0}\right) \mapsto 0 & c\left(\Gamma_{1}\right) \mapsto c\left(\Gamma_{-}\right) & c\left(\Gamma_{2}\right) \mapsto c\left(\Gamma_{-}\right), \\
\Phi_{c}: c\left(\Gamma_{0}\right) \mapsto c\left(\Gamma_{+}\right) & c\left(\Gamma_{1}\right) \mapsto 0 & c\left(\Gamma_{2}\right) \mapsto c\left(\Gamma_{+}\right) .
\end{array}
$$



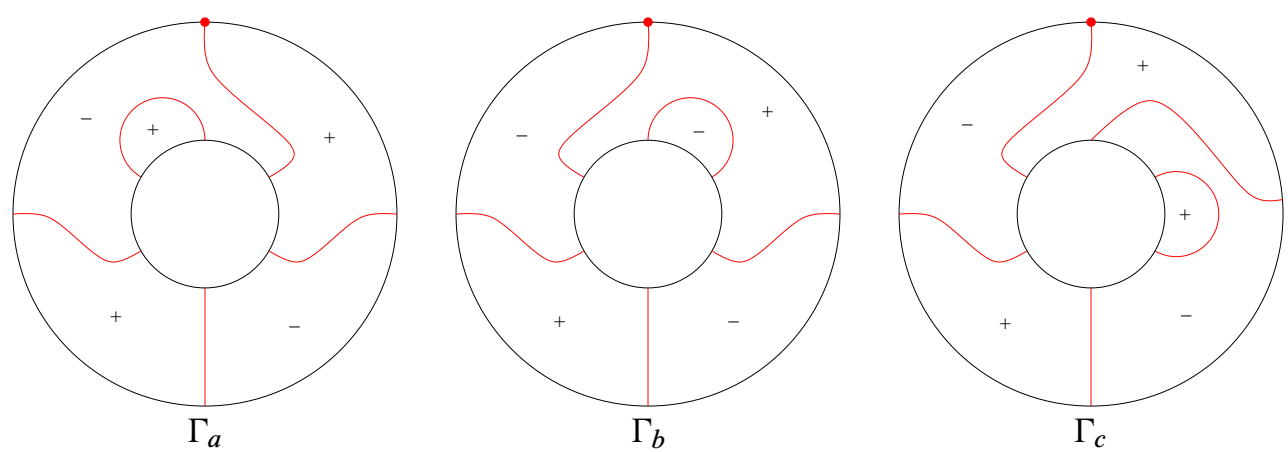

Figure 4: Gluing annuli $\Phi_{a}, \Phi_{b}, \Phi_{c}$
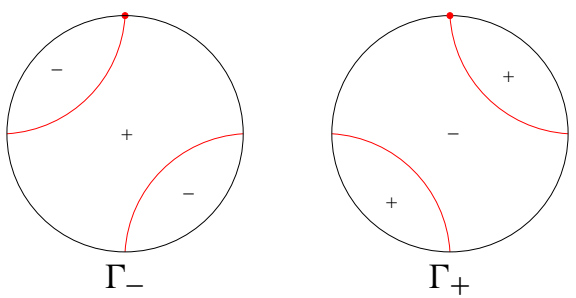

Figure 5: Sutures $\Gamma_{-}, \Gamma_{+}$

As $c\left(\Gamma_{0}\right), c\left(\Gamma_{1}\right), c\left(\Gamma_{2}\right)$ are linearly dependent, let $\alpha c\left(\Gamma_{0}\right)+\beta c\left(\Gamma_{1}\right)+\gamma c\left(\Gamma_{2}\right)=0$ for some $\alpha, \beta, \gamma \in \mathbb{Z}$, not all zero. From $\Phi_{a}, \Phi_{b}, \Phi_{c}$ we then obtain $(\alpha+\beta) c\left(\Gamma_{+}\right)=0$, $(\beta+\gamma) c\left(\Gamma_{-}\right)=0$, and $(\gamma+\alpha) c\left(\Gamma_{+}\right)=0$ respectively. We may then include $\left(D^{2}, F_{2}\right) \hookrightarrow\left(D^{2}, F_{1}\right)$ in various ways so that $\Gamma_{+}$or $\Gamma_{-}$becomes the vacuum $\Gamma_{\varnothing}$. Thus $(\alpha+\beta) c\left(\Gamma_{\varnothing}\right)=(\beta+\gamma) c\left(\Gamma_{\varnothing}\right)=(\gamma+\alpha) c\left(\Gamma_{\varnothing}\right)=0$. As $c\left(\Gamma_{\varnothing}\right)$ is nontorsion, we have either $\alpha+\beta=\beta+\gamma=\gamma+\alpha=0$ or $c\left(\Gamma_{\varnothing}\right)=0$. In the first case $\alpha=\beta=\gamma=0$, a contradiction. Thus $c\left(\Gamma_{\varnothing}\right)=0$; now by inclusion every suture element is 0 .

Return now to our original axioms.

Proposition 3.10 Let $\Gamma$ be a set of sutures on $\left(D^{2}, F_{n}\right)$. If $\Gamma$ contains a closed loop then $c(\Gamma)=\{0\}$. Otherwise, $\Gamma$ is a chord diagram, and $c(\Gamma)$ has two distinct elements and is of the form $\{x,-x\}$.

Proof From Lemma 3.2 for any $\Gamma, c(\Gamma)$ is either a singleton or is of the form $\{x,-x\}$. If $\Gamma$ contains a closed loop then it contains a contractible closed loop, so $c(\Gamma)=\{0\}$. Suppose $\Gamma$ is a chord diagram with $c(\Gamma)$ a singleton. We can find an inclusion map $V\left(D^{2}, F_{n}\right) \longrightarrow V\left(D^{2}, F_{1}\right)$ which takes $c(\Gamma) \mapsto c\left(\Gamma_{\varnothing}\right)$ surjectively; thus $c\left(\Gamma_{\varnothing}\right)$ is a nonzero singleton. As the vacuum includes into any sutures then all $c(\Gamma)$ are singletons, contradicting the previous proposition. 
Note that Proposition 3.10 does not rely on Axiom 9; it relies upon the argument of Proposition 3.9, which in turn relies upon a weaker form of Axiom 9. In particular, Axiom $\left(9^{\prime}\right)$ of Proposition 3.9 is implied by any of the formulations in Proposition 3.7; to see it is implied by any of Axioms (i)-(iv) we use the argument of Lemma 3.5. Thus, we have the following, which we shall need later.

Lemma 3.11 Assume Axioms 1-8 of sutured TQFT and any of the 8 alternative formulations of Axiom 9 in Proposition 3.7. Let $\Gamma$ be any chord diagram. Then $c(\Gamma)$ contains precisely two elements and is of the form $\{x,-x\}$.

As for gluing maps, these necessarily have ambiguity too. Let $\tau$ be a gluing on a sutured background surface $(\Sigma, F)$. Axiom 3 gives at least one map $\Phi_{\tau}$; Axiom 7 requires that $\Phi_{\tau}$ takes $c(\Gamma) \rightarrow c\left(\#_{\tau} \Gamma\right)$ surjectively. By the above, at least on discs, $-\Phi_{\tau}$ will have the same properties. Thus there is no canonical such $\Phi_{\tau}$; we may freely switch signs.

\subsection{Creation and annihilation operators}

We now use gluing/inclusion to define various operators in sutured TQFT. We generally follow [17], though with some notational differences.

Creation operators are maps $V\left(D^{2}, F_{n}\right) \longrightarrow V\left(D^{2}, F_{n+1}\right)$; they include a disc into a larger disc and have the effect of inserting a new outermost chord into a chord diagram in a specific place. Annihilation operators are maps $V\left(D^{2}, F_{n}\right) \longrightarrow V\left(D^{2}, F_{n-1}\right)$, including a disc into a larger disc, with the effect of joining two specific adjacent endpoints of a chord diagram. Each operator is defined by giving a specific sutured annulus. To define each operator, we choose a gluing map $\Phi_{\tau}^{i}$, and a suture element for the sutures on the annulus. This requires careful choices of signs, which we defer to Section 3.8 below; for now we simply consider the effect on sutures.

Recall from Section 3.1 the notation for sutures on $\left(D^{2}, F_{n+1}\right)$, including $n=$ $n_{-}+n_{+}, e=n_{+}-n_{-}$, base and root, eastside and westside. Annihilation operators $a_{ \pm, i}: V\left(D^{2}, F_{n+1}\right) \longrightarrow V\left(D^{2}, F_{n}\right)$, for $0 \leq i \leq n_{ \pm}+1$, have the following effect:

(i) (-)-annihilations: For $0 \leq i \leq n_{-}+1, a_{-, i}$ closes off the region between $(-2 i,-2 i+1)$.

(ii) (+)-annihilations: For $0 \leq i \leq n_{+}+1, a_{+, i}$ closes off the region between $(2 i-1,2 i)$

Note every $( \pm)$-annihilation closes off a $(\mp)$-region; initial annihilations $a_{-, 0}, a_{+, 0}$ close off regions at the basepoint (Figure 6 shows where the basepoint goes); final 
annihilations $a_{-, n_{-}+1}, a_{+, n_{+}+1}$ close off regions at the root point. Internal (-)annihilations $a_{-, i}, 1 \leq i \leq n_{-}$close off regions on the westside; internal $(+)-$ annihilations $a_{+, i}, 1 \leq i \leq n_{+}$close off regions on the eastside. (This numbering of annihilations is different from [17].)
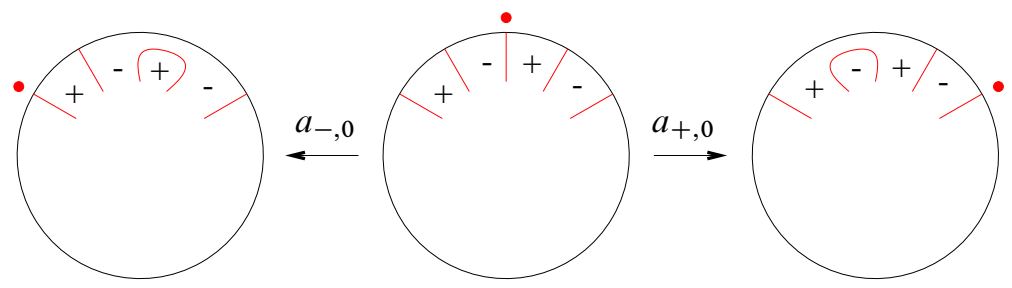

Figure 6: Annihilation maps $a_{ \pm, 0}$

Similarly creation operators $V\left(D^{2}, F_{n+1}\right) \rightarrow V\left(D^{2}, F_{n+2}\right)$ are as follows.

(i) (-)-creations: For $0 \leq i \leq n_{-}+1, a_{-, i}^{*}$ creates a new chord which joins $(-2 i-1,-2 i)$, between the points previously labelled $(-2 i,-2 i+1)$.

(ii) (+)-creations: For $0 \leq i \leq n_{+}+1, a_{+, i}^{*}$ creates a new chord which joins $(2 i, 2 i+1)$, between the points previously labelled $(2 i-1,2 i)$.

Every $( \pm)$-creation creates a new outermost chord enclosing a \pm region; initial creations create new chords at the basepoint (see Figure 7); final creations create new chords at the root point; $(-)$-creations (resp. $(+))$ create new chords on the westside (resp. eastside) of the resulting diagram.
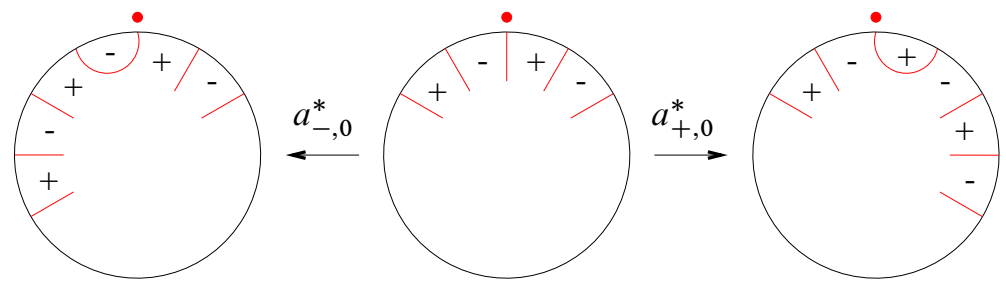

Figure 7: Creation maps $a_{ \pm, 0}^{*}$

It's easy to check that these creation and annihilation operators obey the same relations as in Section 2.2, substituting $(-,+)$ for $(x, y)$, at least up to sign. In Sections 3.8-3.9 we will consider signs carefully and show the relations hold. 


\subsection{Basis, partial order}

Let $\mathcal{W}$ denote the set of words on $\{-,+\}$. In a word, let $n_{ \pm}$denote the number of \pm signs, $n$ the total length, and $e=n_{+}-n_{-}$; so $\left(n, e, n_{-} . n_{+}\right)$are related as $\left(n, e, n_{x}, n_{y}\right)$. Let $\mathcal{W}_{n}$ be the words of length $n$, and $\mathcal{W}_{n}^{e}=\mathcal{W}_{n_{-}, n_{+}}$for words with $n_{ \pm} \pm$signs. Note $\mathcal{W} \cong \mathcal{M}$ as graded monoids, identifying $(-,+) \sim(x, y)$. The partial order $\leq$ carries naturally over to $\mathcal{W}$.

In [17], we defined a distinguished chord diagram $\Gamma_{w}$ on $\left(D^{2}, F_{n+1}\right)$ for each word $w \in \mathcal{W}_{n} ; \Gamma_{w}$ is obtained from the vacuum by applying the sequence of initial creation operators $a_{ \pm, 0}^{*}$ corresponding to $w$; its Euler class is $e=n_{+}-n_{-}$. We showed [17, Section 6.3.2] that creation and annihilation operators act on basis diagrams $\Gamma_{w}$ exactly as corresponding creation and annihilation operators act on words $w$. That is, defining $a_{ \pm, i}$ and $a_{ \pm, i}^{*}$ to act on words in $\{-,+\}$ in $\mathcal{W}_{n}^{e}$, analogously as on words in $\{x, y\}$ in $\mathcal{M}_{n}^{e}$, then for a basis diagram $\Gamma_{w}$, we have $a_{ \pm, i}^{*} \Gamma_{w}=\Gamma_{a_{ \pm, i}^{*} w}$ and $a_{ \pm, i} \Gamma_{w}=\Gamma_{a_{ \pm, i} w}$ (provided $a_{ \pm, i} w \neq 0$; if $a_{ \pm, i} w=0$ then $a_{ \pm, i} \Gamma_{w}$ contains a closed loop).

We showed [17, Proposition 1.16] that over $\mathbb{Z}_{2}$ coefficients, the $c\left(\Gamma_{w}\right)$ form a basis for $S F H_{\text {comb }}(T, n+1)$ : a basis found by applying creation operators to the vacuum. These arguments immediately carry over to sutured TQFT (over $\mathbb{Z}_{2}$ or $\mathbb{Z}$ ), showing that representatives of $c\left(\Gamma_{w}\right)$ form a basis for $V\left(D^{2}, F_{n+1}\right)$, and proving Proposition 3.6; we briefly rerun these arguments here.

For every $w \in \mathcal{W}_{n}$, arbitrarily choose $c_{w} \in c\left(\Gamma_{w}\right)$ (which has $n+1$ chords). To see the $c_{w}$ are linearly independent, suppose some linear combination is zero, $\sum k_{i} c_{w_{i}}=0$. Then we can apply annihilation operators to reduce $\Gamma_{w_{1}}$ to $\Gamma_{\varnothing}$ but every other $\Gamma_{w_{i}}$ to a set of sutures containing a closed curve; we obtain $k_{1} c\left(\Gamma_{\varnothing}\right)=0$, a contradiction. To see the $c_{w}$ span $V\left(D^{2}, F_{n+1}\right)$, observe that any chord diagram $\Gamma$ either has an outermost chord at the basepoint, or is part of a bypass triple in which the other two chord diagrams have outermost chords at the basepoint; thus any element of $c(\Gamma)$ is a linear combination of suture elements for chord diagrams with outermost chords at the basepoint. But a chord diagram $\Gamma$ with an outermost chord at the basepoint satisfies $c(\Gamma)=a_{ \pm, 0}^{*} c\left(\Gamma^{\prime}\right)$ for some chord diagram $\Gamma^{\prime}$ with fewer chords. Applying this observation repeatedly expresses any suture element of a chord diagram as a linear combination of the $c_{w}$. As suture elements span $V\left(D^{2}, F_{n+1}\right)$, the $c_{w}$ span as desired. This argument works over $\mathbb{Z}$ or $\mathbb{Z}_{2}$. (Over $\mathbb{Z}_{2}$ it is now easy to construct an isomorphism $S F H_{\text {comb }}(T, n) \longrightarrow V\left(D^{2}, F_{n}\right)$, proving Proposition 3.6.)

We may refine this argument by Euler class. Since bypass surgery preserves $e$, every $\Gamma_{w}$ in the decomposition of $\Gamma$ satisfies $w \in \mathcal{W}_{n}^{e}$. As in Section 2, write $V\left(D^{2}\right)_{n}^{e}$ or $V\left(D^{2}\right)_{n_{-}, n_{+}}$for the submodule of $V\left(D^{2}, F_{n+1}\right)$ spanned by suture elements of chord diagrams with Euler class $e$; also write $V\left(D^{2}\right)_{n}=V\left(D^{2}, F_{n+1}\right)$, so 
$V\left(D^{2}\right)=\bigoplus_{n} V\left(D^{2}\right)_{n}$ and $V\left(D^{2}\right)_{n}=\bigoplus_{e} V\left(D^{2}\right)_{n}^{e}$. The $c_{w}$, over $w \in \mathcal{W}_{n}^{e}$, form a basis for $V\left(D^{2}\right)_{n}^{e}$; hence

$$
\operatorname{dim} V\left(D^{2}\right)_{n}^{e}=\left(\begin{array}{c}
n \\
n_{-}
\end{array}\right)=\left(\begin{array}{c}
n \\
n_{+}
\end{array}\right) .
$$

Thus, as graded abelian groups, $V\left(D^{2}\right) \cong \mathcal{F}$.

Proposition 3.12 For any choice of $c_{w} \in c\left(\Gamma_{w}\right)$ over $w \in \mathcal{W}_{n}^{e},\left\{c_{w}\right\}$ forms a basis for $V\left(D^{2}\right)_{n}^{e}$.

In [17] we considered stacking basis chord diagrams. We showed [17, Proposition 1.28, Section 4.3] that the set of sutures obtained on the sphere (rounded cylinder) by stacking $\Gamma_{w_{0}}, \Gamma_{w_{1}}$ is connected if and only if $w_{0} \leq w_{1}$. We thus have $\left\langle c_{w_{0}} \mid c_{w_{1}}\right\rangle= \pm 1$ if $w_{0} \leq w_{1}$, and 0 otherwise. (In particular, distinct summands $V\left(D^{2}\right)_{n}^{e}$ are orthogonal.) Thus up to sign, the algebraic (in $\mathcal{F}$ ) and topological (in $V\left(D^{2}\right)$ ) versions of $\langle\cdot \mid \cdot\rangle$ agree. In the next sections we consider signs carefully; this will allow us to prove Theorem 3.4, and a much more general isomorphism in Theorem 5.1.

\subsection{Previous results: suture elements $\bmod 2$}

In [17] we effectively considered in detail the structure of suture elements in $V\left(D^{2}, F_{n}\right)$ over $\mathbb{Z}_{2}$. All those arguments carry over here, except with sign ambiguities. We briefly recall some of these arguments and results as they are needed here.

We proved several properties of stacking; these now give us information about $\langle\cdot \mid \cdot\rangle$ up to sign:

- $[17$, Lemma 3.7] For any chord diagram $\Gamma,\langle c(\Gamma) \mid c(\Gamma)\rangle= \pm 1$.

- [17, Lemma 3.9] If $\Gamma^{\prime}$ is obtained from $\Gamma$ by an upwards bypass surgery then $\left\langle c(\Gamma) \mid c\left(\Gamma^{\prime}\right)\right\rangle= \pm 1$ and $\left\langle c\left(\Gamma^{\prime}\right) \mid c(\Gamma)\right\rangle=0$.

- [17, Lemma 3.8] If two chord diagrams $\Gamma, \Gamma^{\prime}$ share a common outermost chord $\gamma$, then $\left\langle c(\Gamma) \mid c\left(\Gamma^{\prime}\right)\right\rangle= \pm\left\langle c(\Gamma-\gamma) \mid c\left(\Gamma^{\prime}-\gamma\right)\right\rangle$.

- Annihilation and creation operators satisfy the adjoint relations of Section 2.5, up to sign: $\left\langle a_{-, i} c\left(\Gamma_{0}\right) \mid c\left(\Gamma_{1}\right)\right\rangle= \pm\left\langle c\left(\Gamma_{0}\right) \mid a_{-, i}^{*} c\left(\Gamma_{1}\right)\right\rangle$ and $\left\langle c\left(\Gamma_{0}\right) \mid a_{+, i} c\left(\Gamma_{1}\right)\right\rangle=$ $\pm\left\langle a_{+, i}^{*} c\left(\Gamma_{0}\right) \mid c\left(\Gamma_{1}\right)\right\rangle$.

We [17, Section 5.1] defined the notions of elementary move on a word, generalised elementary move and nicely ordered sequence of generalised elementary moves. All these moves come in forwards and backwards versions (moving forwards/backwards with respect to $\leq$ ). A forwards elementary move replaces a substring of the form $(-)^{a}(+)^{b}$ 
with $(+)^{b}(-)^{a}$. A generalised forwards elementary move $\mathrm{FE}(i, j)$ moves the $i-$ th - sign (from the left) to the right, past the $j$-th (from the left) + sign, together with all - signs in between. A sequence of forwards generalised elementary moves $\mathrm{FE}\left(i_{1}, j_{1}\right), \ldots, \mathrm{FE}\left(i_{k}, j_{k}\right)$ is nicely ordered if $i_{1}<i_{2}<\cdots<i_{k}$ and $j_{1} \leq j_{2} \leq \cdots \leq j_{k}$. Backwards versions reverse the roles of - and + .

Two basis chord diagrams $\Gamma_{w_{0}}, \Gamma_{w_{1}}$ are bypass related if and only if $w_{0}, w_{1}$ are related by an elementary move [17, Lemmas 5.7-8]. For any words $w_{0} \leq w_{1}$, there is a nicely ordered sequence of forwards generalised elementary moves taking $w_{0}$ to $w_{1}$. There is also a set $\operatorname{FBS}\left(w_{0}, w_{1}\right)$ of disjoint upwards bypass surgeries on $\Gamma_{w_{0}}$ which effect the moves, eventually giving $w_{1}$. If we instead perform all these bypass surgeries downwards, we obtain a chord diagram $\Gamma$ whose suture element, expressed in terms of the basis, is a sum $(\bmod 2) \sum c\left(\Gamma_{w_{i}}\right)$, where $w_{0}, w_{1}$ occur in the sum, and for every other $w_{i}$ occurring in the sum, $w_{0} \leq w_{i} \leq w_{1}$. In fact these $\Gamma_{w_{i}}$ can be obtained by performing upwards bypass surgeries along subsets of $\operatorname{FBS}\left(w_{0}, w_{1}\right)$. Since the number of pairs $w_{0} \leq w_{1}$ in $\mathcal{W}_{n}^{e}$ [17, Proposition 1.19], and the number of chord diagrams with $n+1$ chords and Euler class $e$, are both the Narayana number $N_{n}^{e}$, all suture elements of chord diagrams are of this form [17, Theorem 1.20].

The same argument applies immediately in sutured TQFT over $\mathbb{Z}$ : there is still a bijection between comparable pairs $w_{0} \leq w_{1}$ and chord diagrams with minimum and maximum words $w_{0}, w_{1}$ occurring in their suture elements. For $\Gamma$ as above and $c \in$ $c(\Gamma)$ we have $c=\sum a_{i} c_{w_{i}}$, where $c_{w_{i}} \in c\left(\Gamma_{w_{i}}\right)$ and the $w_{i}$ all satisfy $w_{0} \leq w_{i} \leq w_{1}$. Following [17], write $\Gamma=\left[\Gamma_{w_{0}}, \Gamma_{w_{1}}\right]$, and $\pm\left[w_{0}, w_{1}\right]$ for the corresponding suture elements. All coefficients $a_{i} \in\{-1,1\}$, as when we decompose $\Gamma$ in terms of the basis, using the decomposition algorithm, any basis diagram occurs at most once. If $\Gamma$ is not a basis diagram, then an even number of basis diagrams appears in its decomposition [17, Proposition 1.23].

\subsection{Choosing a coherent basis}

Although sign ambiguity prevents us choosing canonical representatives for all $c(\Gamma)$ coherently, we will choose representatives for basis suture elements. We do this using creation operators and $\langle\cdot \mid \cdot\rangle$.

Choose a vacuum representative $c_{\varnothing} \in c\left(\Gamma_{\varnothing}\right)$ arbitrarily. For each pair $\left(n_{-}, n_{+}\right)$, choose an arbitrary representative for the creation $a_{+, 0}^{*}: V\left(D^{2}\right)_{n_{-,} n_{+}} \longrightarrow V\left(D^{2}\right)_{n_{-,} n_{+}+1}$. For each $\left(n_{-}, n_{+}\right)=\left(n_{-}, 0\right)$, choose an arbitrary representative for the creation map $a_{-, 0}^{*}: V\left(D^{2}\right)_{n_{-}, 0} \longrightarrow V\left(D^{2}\right)_{n_{-}+1,0}$. For each $\left(n_{-}, n_{+}\right)$there is then a unique sequence of these chosen $a_{-, 0}^{*}$ and $a_{+, 0}^{*}$ operators leading from $V\left(D^{2}\right)_{0,0}$ to $V\left(D^{2}\right)_{n_{-}, n_{+}}$; this sequence of operators takes $c\left(\Gamma_{\varnothing}\right)$ to $c\left(\Gamma_{w_{\max }}\right)$, where $w_{\max }=(+)^{n_{+}}(-)^{n_{-}}$is 
the maximum word in $\mathcal{W}_{n_{-}, n_{+}}$. For each $\left(n_{-}, n_{+}\right)$, choose $c_{w_{\max }} \in c\left(\Gamma_{w_{\max }}\right) \subset$ $V\left(D^{2}\right)_{n_{-}, n_{+}}$to be the image of $c_{\varnothing}$ under these creations.

We noted (Section 3.6) that the summands $V\left(D^{2}\right)_{n}^{e}$ are $\langle\cdot \mid \cdot\rangle$-orthogonal. Thus we may write $\langle\cdot \mid \cdot\rangle_{n}^{e}$ for stacking in $V\left(D^{2}\right)_{n}^{e}$; adjusting $\langle\cdot \mid \cdot\rangle_{n}^{e}$ by a sign preserves all required properties of $\langle\cdot \mid \cdot\rangle$.

In each $V\left(D^{2}\right)_{n}^{e}$ we have chosen $c_{w_{\max }}$. We have $\left\langle c_{w_{\max }} \mid c_{w_{\max }}\right\rangle= \pm 1$. The form $\langle\cdot \mid \cdot\rangle$ is now fixed by adjusting each $\langle\cdot \mid \cdot\rangle_{n}^{e}$ by a sign if necessary so that

$$
\left\langle c_{w_{\max }} \mid c_{w_{\max }}\right\rangle=1 .
$$

For each $w \in \mathcal{W}_{n}^{e}$ and $c_{w} \in c\left(\Gamma_{w}\right)$, we have $\left\langle c_{w} \mid c_{w_{\max }}\right\rangle= \pm 1$; we choose, once and for all, the representative $c_{w}$ such that

$$
\left\langle c_{w} \mid c_{w_{\max }}\right\rangle=1 .
$$

We next show this basis is coherent.

\subsection{Coherent creation and annihilation}

Having chosen signs on some initial creation operators (arbitrarily), we now choose representatives for all creation and annihilation operators, so that they are coherent with respect to the basis $c_{w}$.

We have $a_{ \pm, i}^{*} c_{w}= \pm c_{a_{ \pm, i}^{*} w}$ and $a_{ \pm, i} c_{w}= \pm c_{a_{ \pm, i} w}$ (where we set $c_{0}=0$ ). In $\mathcal{W}_{n}^{e}$ we have the minimal and maximal words $w_{\min }=(-)^{n_{-}}(+)^{n_{+}}, w_{\max }=(+)^{n_{+}}(-)^{n_{-}}$. We choose each creation operator $a_{ \pm, i}^{*}$ to take $c_{w_{\max }} \mapsto c_{a_{ \pm, i}^{*} w_{\max }}$; and we choose each annihilation operator $a_{ \pm, i}$ to take $c_{w_{\max }} \mapsto c_{a_{ \pm, i} w_{\max }}$, unless this is 0 ; else we define it to take $c_{w_{\text {min }}} \mapsto c_{a_{ \pm, i} w_{\text {min }}}$. Clearly this agrees with previous choices of creation operators.

Lemma 3.13 If $w_{0} \leq w_{1}$ are words related by an elementary move, then $c_{w_{0}}-c_{w_{1}}$ is a suture element.

Proof There is an upwards bypass move on $\Gamma_{w_{0}}$ which gives $\Gamma_{w_{1}}$; the corresponding downwards bypass move gives a suture element of the form $c_{w_{0}} \pm c_{w_{1}}$. Considering stacking these, mod 2 we have $\left\langle c_{w_{0}}+c_{w_{1}} \mid c_{w_{\max }}\right\rangle=0$. By our choice of basis, $\left\langle c_{w_{0}} \mid c_{w_{\max }}\right\rangle=\left\langle c_{w_{1}} \mid c_{w_{\max }}\right\rangle=1$. Thus $\left\langle c_{w_{0}} \pm c_{w_{1}} \mid c_{w_{\max }}\right\rangle=1 \pm 1$; the result must be 0 , and $c_{w_{0}}-c_{w_{1}}$ is a suture element.

Lemma 3.14 For any $w,\left\langle c_{w} \mid c_{w}\right\rangle=1$. For any two $w_{0} \leq w_{1}$ in $\mathcal{W}_{n}^{e}$ related by an elementary move, $\left\langle c_{w_{0}} \mid c_{w_{1}}\right\rangle=1$. 
Proof We use the following fact:

- If $w_{0} \leq w_{1}$ are related by an elementary move and $\left\langle c_{w_{1}} \mid c_{w_{1}}\right\rangle=1$, then $\left\langle c_{w_{0}} \mid c_{w_{0}}\right\rangle=\left\langle c_{w_{0}} \mid c_{w_{1}}\right\rangle=1$.

To see why the fact is true, from the previous lemma $c_{w_{0}}-c_{w_{1}}$ is a suture element. We have mod 2 (hence over $\mathbb{Z}$ ) $\left\langle c_{w_{0}}-c_{w_{1}} \mid c_{w_{1}}\right\rangle=0$, so that $\left\langle c_{w_{0}} \mid c_{w_{1}}\right\rangle=\left\langle c_{w_{1}} \mid c_{w_{1}}\right\rangle=1$. Then $\left\langle c_{w_{0}} \mid c_{w_{0}}-c_{w_{1}}\right\rangle=0$ so $\left\langle c_{w_{0}} \mid c_{w_{0}}\right\rangle=\left\langle c_{w_{0}} \mid c_{w_{1}}\right\rangle=1$.

By our choice of $\langle\cdot \mid \cdot\rangle,\left\langle c_{w_{\max }} \mid c_{w_{\max }}\right\rangle=1$. Using the fact repeatedly, we obtain the desired result.

Proposition 3.15 (Coherence of creation and annihilation) For any $w \in \mathcal{W}_{n}^{e}, s \in$ $\{-,+\}$ and $0 \leq i \leq n_{s}+1$,

$$
a_{s, i}^{*} c_{w}=c_{a_{s, i}^{*} w} \quad \text { and } \quad a_{s, i} c_{w}=c_{a_{s, i} . w} .
$$

Proof Consider an annihilation $a_{s, i}$, and let $A \subset \mathcal{W}_{n}^{e}$ consist of words $w$ for which $a_{s, i} c_{w}=c_{a_{s, i} w}$. By definition of $a_{s, i}, A$ is nonempty $\left(w_{\max }\right.$ or $\left.w_{\min } \in A\right)$. Now repeatedly apply the following fact.

- If $w \in A$ and $w^{\prime}$ is obtained from $w$ by an elementary move, then $w^{\prime} \in A$.

To see why this fact is true, note (by Lemma 3.13) $c_{w^{\prime}}-c_{w}$ is a suture element, hence is taken by $a_{s, i}$ to a suture element (possibly 0$), a_{s, i}\left(c_{w^{\prime}}-c_{w}\right)= \pm c_{a_{s, i} w^{\prime}}-c_{a_{s, i} w}$. As $a_{s, i} w^{\prime}$ and $a_{s, i} w$ are related by an elementary move, or are identical, $c_{a_{s, i} w^{\prime}}-c_{a_{s, i} w}$ is a suture element (possibly 0 ) and the other alternative is not. Thus $w^{\prime} \in A$.

The argument for creation operators is similar (and simpler; the 0 case does not arise).

\section{Proposition 3.16 (Coherence of basis)}

(i) For words $w_{0}, w_{1} \in \mathcal{W}_{n}^{e}$ with $w_{0} \leq w_{1},\left\langle c_{w_{0}} \mid c_{w_{1}}\right\rangle=1$.

(ii) For any chord diagram $\Gamma$ and $c \in c(\Gamma), c=\sum_{w} a_{w} c_{w}$ where $a_{w} \in\{-1,1\}$. If $\Gamma$ is not a basis chord diagram then $\sum a_{w}=0$.

(iii) For each chord diagram $\Gamma$ and suture element $c \in c(\Gamma) \subset V\left(D^{2}\right)_{n}^{e},\langle c \mid c\rangle=1$.

Proof We first prove (ii). In Section 3.7 we showed that all $a_{w}= \pm 1$; we only need show $\sum a_{w}=0$ when $\Gamma$ is a nonbasis diagram. The easiest way to see this is to use the fact, shown in $\left[17\right.$, Section 5.2.1], that if $\Gamma$ is a nonbasis diagram then $\Gamma, \Gamma_{w_{\max }}$ are not stackable, $\left\langle c(\Gamma) \mid c\left(\Gamma_{w_{\max }}\right)\right\rangle=0$. Take $\sum_{w} a_{w} c_{w}=c \in c(\Gamma)$. Since the $c_{w}$ by definition satisfy $\left\langle c_{w} \mid c_{w_{\max }}\right\rangle=1$ we obtain $\sum_{w} a_{w}=0$. 
Next we prove (iii). We will repeatedly apply this fact:

- Let $x \in c\left(\Gamma_{x}\right), y \in c\left(\Gamma_{y}\right)$, where $\Gamma_{y}$ is a chord diagram obtained from $\Gamma_{x}$ by bypass surgery (without loss of generality, upwards), so $\langle x \mid y\rangle= \pm 1$ and $\langle y \mid x\rangle=0$. Suppose $\langle x \mid x\rangle=\langle y \mid y\rangle=1$. Change sign of $y$ if necessary so that $\langle x \mid y\rangle=1$. Then $x-y$ is a suture element and $\langle x-y \mid x-y\rangle=1$.

To see why, note there is a suture element of the form $x \pm y$. We have $\langle x \pm y \mid x \pm y\rangle=$ $2 \pm\langle x \mid y\rangle$; of course we must have $2-1=1$, and the conclusions follow.

Consider the set $A$ of suture elements $c$ such that $\langle c \mid c\rangle=1$. We know (Lemma 3.14) that $A$ contains all $\pm c_{w}$, and (by the above fact) if it contains two suture elements for chord diagrams related by a bypass surgery, then it contains the suture element for the third chord diagram in their bypass triple. Repeated application of the above fact (eg considering each step of a decomposition of a chord diagram into basis diagrams) gives (iii).

We now prove (i). Suppose there exist $w_{0} \leq w_{1}$ such that $\left\langle c_{w_{0}} \mid c_{w_{1}}\right\rangle \neq 1$, that is, $\left\langle c_{w_{0}} \mid c_{w_{1}}\right\rangle=-1$. Fix $w_{0}$ and take $w_{1}$ minimal among such pairs (so if $w_{0} \leq w<w_{1}$ then $\left\langle c_{w_{0}} \mid c_{w}\right\rangle=1$ ). Since (Lemma 3.14) $\left\langle c_{w_{0}} \mid c_{w_{0}}\right\rangle=1, w_{0} \neq w_{1}$. Now take the chord diagram $\Gamma=\left[\Gamma_{w_{0}}, \Gamma_{w_{1}}\right]$ and $c \in c(\Gamma)$. As $w_{0} \neq w_{1}, \Gamma$ is not a basis diagram. Decompose $c=\sum_{w} a_{w} c_{w}$, so all $a_{w}= \pm 1$ and $\sum_{w} a_{w}=0$. We have

$$
\left\langle c_{w_{0}} \mid c\right\rangle=\left\langle c_{w_{0}} \mid \sum_{w} a_{w} c_{w}\right\rangle=\sum_{w} a_{w}\left\langle c_{w_{0}} \mid c_{w}\right\rangle .
$$

Since all $w$ occurring satisfy $w_{0} \leq w \leq w_{1}, \bmod 2$ we have $\left\langle c_{w_{0}} \mid c\right\rangle=\sum_{w} a_{w}=0$; hence the same is true over $\mathbb{Z}$. On the other hand, all $\left\langle c_{w_{0}} \mid c_{w}\right\rangle=1$ except for $\left\langle c_{w_{0}} \mid c_{w_{1}}\right\rangle=-1$. So

$$
\sum_{w} a_{w}=0 \quad \text { and } \quad-a_{w_{1}}+\sum_{w \neq w_{1}} a_{w}=0 .
$$

As these two sums differ by $2 a_{w_{1}} \neq 0$, we have a contradiction.

\subsection{Multiplication}

Given two chord diagrams $\Gamma_{0}, \Gamma_{1}$ on $\left(D^{2}, F_{n_{0}+1}\right),\left(D^{2}, F_{n_{1}+1}\right)$ with Euler classes $e_{0}, e_{1}$, we now multiply them. Consider a gluing $\tau$ which identifies a neighbourhood of the root point on $\partial\left(D^{2}, F_{n_{0}}\right)$ with a neighbourhood of the basepoint on $\partial\left(D^{2}, F_{n_{1}}\right)$. Taking a gluing map for $\tau$ and restricting to $e_{0}, e_{1}$ summands gives a linear operator

$$
\times\left(n_{0}, e_{0}\right),\left(n_{1}, e_{1}\right): V\left(D^{2}\right)_{n_{0}}^{e_{0}} \otimes V\left(D^{2}\right)_{n_{1}}^{e_{1}} \longrightarrow V\left(D^{2}\right)_{n_{0}+n_{1}}^{e_{0}+e_{1}} .
$$


Note $\times$ acts on basis suture elements by multiplying the corresponding words:

$$
c\left(\Gamma_{w_{0}}\right) \otimes c\left(\Gamma_{w_{1}}\right) \mapsto c\left(\Gamma_{w_{0} w_{1}}\right) .
$$

Taking $\times=\bigoplus_{n_{0}, e_{0}, n_{1}, e_{1}} \times\left(n_{0}, e_{0}\right),\left(n_{1}, e_{1}\right)$ we obtain a $\mathbb{Z}$-bilinear associative multiplication, making $V\left(D^{2}\right)$ a bigraded ring. There are sign ambiguities in $\times$; we now resolve them, adjusting each $\times_{\left(n_{0}, e_{0}\right),\left(n_{1}, e_{1}\right)}$ individually. Choose $\times_{\left(n_{0}, e_{0}\right),\left(n_{1}, e_{1}\right)}$ to send maximal basis elements to basis elements, ie

$$
c_{(+)^{n_{+, 0}}(-)^{n_{-, 0}} \otimes} \otimes c_{(+)^{n_{+, 1}}(-)^{n_{-, 1}}} \mapsto c_{(+)^{n_{+, 0}}(-)^{n_{-, 0}}(+)^{n_{+, 1}}(-)^{n_{-, 1}}}
$$

where $n_{+, i}, n_{-, i}$ is the pair $n_{+}, n_{-}$corresponding to $\left(n_{i}, e_{i}\right)$. All multiplication respects the basis; the method of proof is by now familiar.

Proposition 3.17 For any $w_{0} \in \mathcal{W}_{n_{0}}^{e_{0}}$ and $w_{1} \in \mathcal{W}_{n_{1}}^{e_{1}}$, under $\times_{\left(n_{0}, e_{0}\right),\left(n_{1}, e_{1}\right)}$,

$$
c_{w_{0}} \otimes c_{w_{1}} \mapsto c_{w_{0} w_{1}} .
$$

Proof Consider the set $A$ of words for which this multiplication is coherent: $A=$ $\left\{\left(w_{0}, w_{1}\right): c_{w_{0}} \otimes c_{w_{1}} \mapsto c_{w_{0} w_{1}}\right\}$. By our sign choice of $\times_{\left(n_{0}, e_{0}\right),\left(n_{1}, e_{1}\right)}$, the pair of maximum words $\left((+)^{n_{+, 0}}(-)^{n_{-, 0}},(+)^{n_{+, 1}}(-)^{n_{-, 1}}\right) \in A$. The result now follows obviously from the following two facts.

- If $\left(w_{0}, w_{1}\right) \in A$ and $w_{0}^{\prime}$ is related to $w_{0}$ by an elementary move, then $\left(w_{0}^{\prime}, w_{1}\right) \in A$.

- If $\left(w_{0}, w_{1}\right) \in A$ and $w_{1}^{\prime}$ is related to $w_{1}$ by an elementary move, then $\left(w_{0}, w_{1}^{\prime}\right) \in A$.

To see why the first fact is true, note that $c_{w_{0}^{\prime}}-c_{w_{0}}$ is a suture element, and multiplication must take $\left(c_{w_{0}^{\prime}}-c_{w_{0}}\right) \otimes w_{1}$ to a suture element. The result of this multiplication must be $\pm c_{w_{0}^{\prime} w_{1}}-c_{w_{0} w_{1}}$. But $w_{0}^{\prime} w_{1}$ and $w_{0} w_{1}$ are related by an elementary move, hence $c_{w_{0}^{\prime} w_{1}}-c_{w_{0} w_{1}}$ is a suture element and $-c_{w_{0}^{\prime} w_{1}}-c_{w_{0} w_{1}}$ is not. So $c_{w_{0}^{\prime}} \otimes c_{w_{1}} \mapsto c_{w_{0}^{\prime} w_{1}}$ and $\left(w_{0}^{\prime}, w_{1}\right) \in A$. The second fact is similar.

\subsection{Temperley-Lieb algebra}

Consider the two creation operators $a_{+, i}^{*}$ and $a_{+, i+1}^{*}$, acting $V\left(D^{2}\right)_{n}^{e} \longrightarrow V\left(D^{2}\right)_{n+1}^{e+1}$, for $0 \leq i \leq n_{+}$. Both are obtained by gluing a sutured annulus to the exterior of a disc. Observe that these two sets of sutures are bypass-related. Take the third set of sutures of this bypass triple; gluing these to the exterior of a disc gives an operator (see Figure 8):

$$
T_{+, i}^{*}: V\left(D^{2}\right)_{n}^{e} \longrightarrow V\left(D^{2}\right)_{n+1}^{e+1}
$$


Similarly on the westside, consider $a_{-, i}^{*}$ and $a_{-, i+1}^{*}$, for $0 \leq i \leq n_{-}$, which give annuli with bypass-related sutures, and obtain

$$
T_{-, i}^{*}: V\left(D^{2}\right)_{n}^{e} \longrightarrow V\left(D^{2}\right)_{n+1}^{e-1} .
$$

Annihilation operators $a_{ \pm, i}, a_{ \pm, i+1}\left(0 \leq i \leq n_{ \pm}\right)$also give annuli with bypass-related
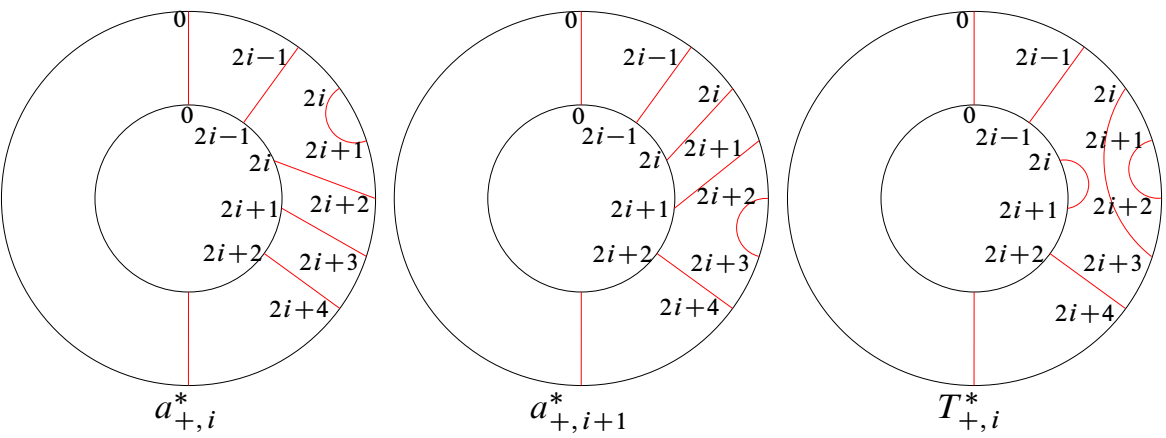

Figure 8: Operators $a_{+, i}^{*}, a_{+, i+1}^{*}$ and $T_{+, i}^{*}$

sutures, and taking a sutured annulus forming a bypass triple with them, we obtain gluing maps

$$
T_{ \pm, i}: V\left(D^{2}\right)_{n}^{e} \longrightarrow V\left(D^{2}\right)_{n-1}^{e \pm 1} .
$$

These gluing maps are ambiguous up to sign; we choose coherent signs. First, $T_{+, i}^{*}$ takes $c_{w}$ to $\pm c_{a_{+, i}^{*} w} \pm c_{a_{+, i+1}^{*} w}$, and $a_{+, i}^{*} w, a_{+, i+1}^{*} w$ are words related by an elementary move, or are identical; hence $\pm\left(c_{a_{+, i}^{*} w}-c_{a_{+, i+1}^{*} w}\right)$ are suture elements (and the other possibilities are not). Similar considerations apply to $T_{-, i}^{*}$. Thus we set

$$
T_{ \pm, i}^{*}=a_{ \pm, i}^{*}-a_{ \pm, i+1}^{*} .
$$

Similarly, $T_{+, i}$ takes $c_{w}$ to $\pm c_{a_{+, i} w} \pm c_{a_{+, i+1}} w$, and the two words $a_{+, i} w, a_{+, i+1} w$ are either identical or related by an elementary move; thus we define

$$
T_{ \pm, i}=a_{ \pm, i}-a_{ \pm, i+1} .
$$

These $T_{ \pm, i}, T_{ \pm, i}^{*}$ are identical to the operators of the same name in $\mathcal{F}$; we may define

$$
U_{ \pm, i}=T_{ \pm, i}^{*} a_{ \pm, i+1} .
$$

so that $U_{ \pm, i}$ are operators $V\left(D^{2}\right)_{n}^{e} \longrightarrow V\left(D^{2}\right)_{n}^{e}$ which glue annuli to the exterior of discs, having the effect shown in Figure 9. We see then (Figure 10) that the $U_{ \pm, i}$ have sutures which are very similar to the usual generators given for the Temperley-Lieb algebra. 

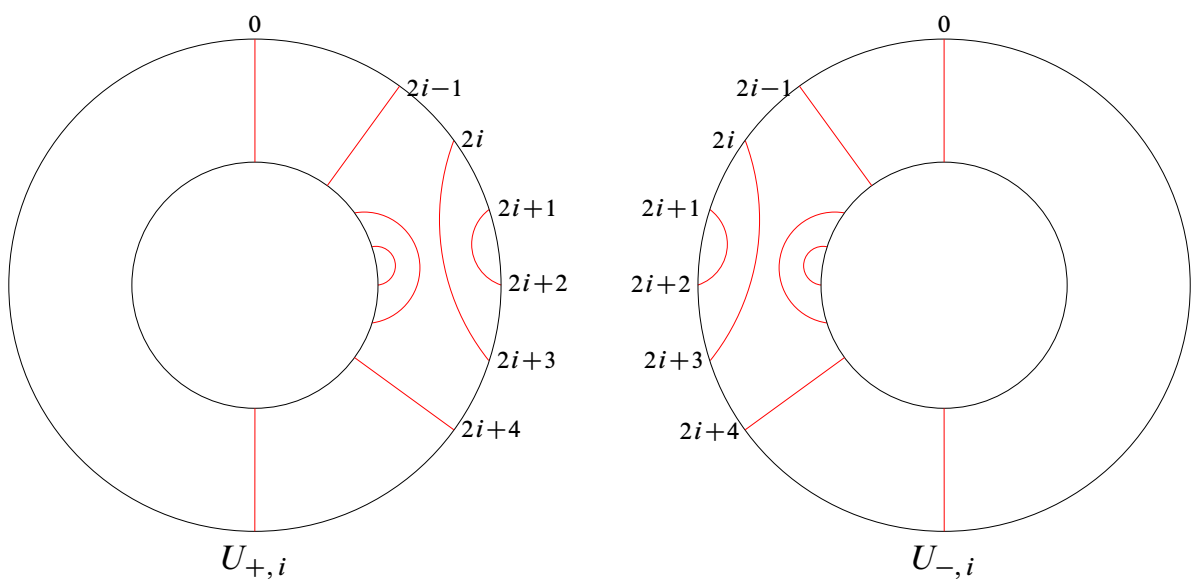

Figure 9: Operators $U_{+, i}, U_{-, i}$

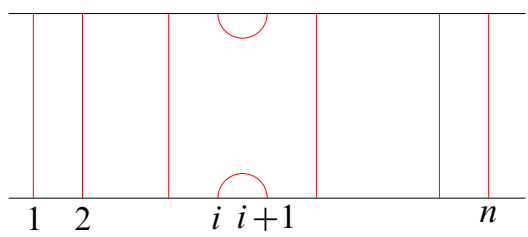

Figure 10: Usual generators $U_{i}$ in Temperley-Lieb algebra

It follows immediately that the $U_{ \pm, i}$ obey the relations of the Temperley-Lieb algebra (with $\delta=0$ ), up to sign. Since the $U_{ \pm, i}$ are identical algebraically to the operators of Section 2.8, we have the "twisted" representation of the Temperley-Lieb algebra described there.

\subsection{Rotation}

The operation of rotating a chord diagram gives an operation in sutured TQFT. As in [17, Section 7.1], gluing a sutured annulus, with sutures as in Figure 11, to the exterior of a disc has the effect of rotating the basepoint two places (which preserves signs on either side of the basepoint.) We obtain an operator, up to sign:

$$
R: V\left(D^{2}\right)_{n}^{e} \longrightarrow V\left(D^{2}\right)_{n}^{e}
$$

Consider computing $\left\langle c\left(\Gamma_{0}\right) \mid c\left(\Gamma_{1}\right)\right\rangle$, placing two chord diagrams into the ends of a cylinder and rounding sutures. We see that $\left\langle c\left(\Gamma_{0}\right) \mid c\left(\Gamma_{1}\right)\right\rangle=\left\langle c\left(\Gamma_{1}\right) \mid R c\left(\Gamma_{0}\right)\right\rangle$; the rotation compensates precisely for the difference in rounding corners when the ends of the cylinder are swapped. For $c_{0} \in c\left(\Gamma_{0}\right), c_{1} \in c\left(\Gamma_{1}\right)$ then $\left\langle c_{0} \mid c_{1}\right\rangle= \pm\left\langle c_{1} \mid R c_{0}\right\rangle$, similar to the duality property of $H$ on $\mathcal{F}$. 


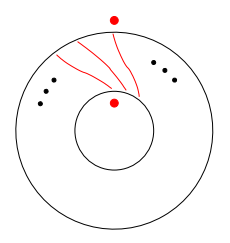

Figure 11: The rotation operator

We choose a sign for $R$ by noting that for any $n, e, R$ takes the basis chord diagram $\Gamma_{w_{\min }}$ to $\Gamma_{w_{\max }}$; we choose a sign on $R$ so that $R c_{w_{\min }}=c_{w_{\max }}$.

Proposition 3.18 With this choice of sign, $R=H$.

Proof We first claim that for all $w \in \mathcal{W}_{n}^{e},\left\langle c_{w} \mid R c_{w}\right\rangle=1$. Let $A=\left\{w \in \mathcal{W}_{n}^{e}\right.$ : $\left.\left\langle c_{w} \mid R c_{w}\right\rangle=1\right\}$. Since $R c_{w_{\min }}=c_{w_{\max }}, w_{\min } \in A$. The claim then follows obviously from the following fact:

- If $w_{0} \in A, w_{0} \leq w_{1}$ and $w_{0}, w_{1}$ are related by an elementary move, then $w_{1} \in A$.

To see why the fact is true, note $c_{w_{0}}-c_{w_{1}}$ is a suture element and $\left\langle c_{w_{0}} \mid c_{w_{0}}-c_{w_{1}}\right\rangle=0$. Thus $\left\langle c_{w_{0}}-c_{w_{1}} \mid R c_{w_{0}}\right\rangle=0$. Hence $\left\langle c_{w_{0}} \mid R c_{w_{0}}\right\rangle=\left\langle c_{w_{1}} \mid R c_{w_{0}}\right\rangle$; as $w_{0} \in A$ we have $\left\langle c_{w_{1}} \mid R c_{w_{0}}\right\rangle=1$. Also note $\left\langle c_{w_{0}}-c_{w_{1}} \mid c_{w_{1}}\right\rangle=0$, so $\left\langle c_{w_{1}} \mid R\left(c_{w_{0}}-c_{w_{1}}\right)\right\rangle=0$. Hence $\left\langle c_{w_{1}} \mid R c_{w_{1}}\right\rangle=\left\langle c_{w_{1}} \mid R c_{w_{0}}\right\rangle=1$, so $w_{1} \in A$.

Next we claim that for any words $w_{0}, w_{1},\left\langle c_{w_{0}} \mid c_{w_{1}}\right\rangle=\left\langle c_{w_{1}} \mid R c_{w_{0}}\right\rangle$. This is clearly true when $w_{0} \not \leq w_{1}$; both are 0 . So take $w \in \mathcal{W}_{n}^{e}$ and let $A_{w}=\left\{w^{\prime} \in \mathcal{W}_{n}^{e}:\left\langle c_{w} \mid c_{w^{\prime}}\right\rangle=\right.$ $\left.\left\langle c_{w^{\prime}} \mid R c_{w}\right\rangle\right\}$; we will show $A_{w}=\mathcal{W}_{n}^{e}$. From the previous claim, $w \in A_{w}$; and any $w^{\prime}$ with $w \not \leq w^{\prime}$ lies in $A_{w}$. Thus we only need consider $w^{\prime}$ with $w \leq w^{\prime}$. Such a $w^{\prime}$ can be reached from $w$ by a sequence of forwards elementary moves, and hence the claim follows from the following fact.

- If $w \leq w_{0} \leq w_{1}$, and $w_{0} \in A_{w}$, and $w_{0}, w_{1}$ are related by an elementary move, then $w_{1} \in A_{w}$.

To see this, note $c_{w_{0}}-c_{w_{1}}$ is a suture element, and $\left\langle c_{w} \mid c_{w_{0}}-c_{w_{1}}\right\rangle=0$, hence $\left\langle c_{w_{0}}-c_{w_{1}} \mid R c_{w}\right\rangle=0$. Thus $\left\langle c_{w_{0}} \mid R c_{w}\right\rangle=\left\langle c_{w_{1}} \mid R c_{w}\right\rangle$. As $w_{0} \in A_{w}$ then $\left\langle c_{w_{0}} \mid R c_{w}\right\rangle=$ $\left\langle c_{w} \mid c_{w_{0}}\right\rangle=1$; thus $\left\langle c_{w_{1}} \mid R c_{w}\right\rangle=1=\left\langle c_{w} \mid c_{w_{1}}\right\rangle$.

Thus for any words, $\left\langle c_{w_{0}} \mid c_{w_{1}}\right\rangle=\left\langle c_{w_{1}} \mid R c_{w_{0}}\right\rangle$. As the $c_{w}$ form a basis for $V\left(D^{2}\right)_{n}^{e}$, the result follows. 
It follows immediately that $H^{n+1} c_{w}= \pm c_{w}$ for every $w \in \mathcal{W}_{n}^{e}$, hence $H^{2 n+2}=1$. The precise sign $H^{n+1}=(-1)^{n-n_{+}}$and period (Theorem 2.6) are proved in Section 5.4.

It also follows that $R$ is an isometry: $\langle u \mid v\rangle=\langle R u \mid R v\rangle$. This is obvious up to sign from the definition of $R$; the full equality now clear by definition of $H$.

Recall an $a_{ \pm, i}^{*}$ inserts an outermost chord into a chord diagram at different positions; many different positions, not all. We showed in Section 3.9 that the $a_{ \pm, i}^{*}$ are isometries; they are also clearly injective. We now note that the operation of inserting an outermost chord anywhere gives a linear operator which is an injective isometry: for it can be obtained by composing the injective isometries $R$ and $a_{ \pm, 0}^{*}$.

\subsection{Variations of nondegeneracy axioms}

We now prove Propositions 3.7 and 3.8, regarding variations of Axiom 9. Recall (Section 3.7) that given $w_{0} \leq w_{1}, \pm\left[w_{0}, w_{1}\right]$ denotes the two suture elements with minimal and maximal basis elements $c_{w_{0}}, c_{w_{1}}$. Recall also (Section 2.4) the notions of difference $d\left(w_{0}, w_{1}\right)$, minimum and maximum of words.

We will need the fact that any two words appear together in a suture element.

Lemma 3.19 For any $w_{0}, w_{1} \in \mathcal{W}_{n}^{e}$, both $c_{w_{0}}, c_{w_{1}}$ appear in the basis decomposition of $\pm\left[\min \left(w_{0}, w_{1}\right), \max \left(w_{0}, w_{1}\right)\right]$. Moreover, in the decomposition of Lemma 2.4,

$\pm\left[\min \left(w_{0}, w_{1}\right), \max \left(w_{0}, w_{1}\right)\right]$

$$
= \pm\left[w_{0}^{0}, w_{1}^{0}\right]\left[w_{1}^{1}, w_{0}^{1}\right]\left[w_{0}^{2}, w_{1}^{2}\right]\left[w_{1}^{3}, w_{0}^{3}\right] \cdots\left[w_{1}^{2 k-1}, w_{0}^{2 k-1}\right] .
$$

Proof In each $\left[w_{0}^{i}, w_{1}^{i}\right]$ or $\left[w_{1}^{i}, w_{0}^{i}\right]$, the basis elements $c_{w_{0}^{i}}, c_{w_{1}^{i}}$ each appear and are minimal/maximal. After multiplying, the basis elements $c_{w_{0}}, c_{w_{1}}, c_{\min \left(w_{0}, w_{1}\right)}$, $c_{\max \left(w_{0}, w_{1}\right)}$ all appear with $c_{\min \left(w_{0}, w_{1}\right)}$ minimal and $c_{\max \left(w_{0}, w_{1}\right)}$ maximal.

Proof of Proposition 3.7 The pairs (i)-(ii), (iii)-(iv), (v)-(vi), (vii)-(viii) are equivalent: for sutures $\Gamma$ with closed components, Axiom 6 gives $c(\Gamma)=\{0\}$; for chord diagrams $\Gamma$, Lemma 3.11 (which works for any of the 8 variations) gives $c(\Gamma)=\{x,-x\}$; equivalence of the four pairs follows.

Implications (ii) $\Rightarrow$ (iv) and (vi) $\Rightarrow$ (viii) are obvious. The Inclusion axiom immediately gives (viii) $\Rightarrow($ vi). Lemma 3.5 is precisely (ii) $\Rightarrow(v)$; in fact the argument shows (iv) $\Rightarrow(\mathrm{v})$. We have

$$
\{(\mathrm{i}) \Leftrightarrow \text { (ii) }\} \Rightarrow\{(\text { iii) } \Leftrightarrow \text { (iv) }\} \Rightarrow\{(\mathrm{v}) \Leftrightarrow \text { (vi) } \Leftrightarrow \text { (vii) } \Leftrightarrow \text { (viii) }\} \text {. }
$$


It now suffices to show (v) $\Rightarrow\{(\mathrm{i})$, (ii) $\}$. Assume (v), a bypass relation. In developing the structure of $V\left(D^{2}\right)$ we only used Axiom 9 to prove (v) (Lemma 3.5), and then used (v) repeatedly; we have not used (i) or (ii) independently. Thus, we obtain all the structure described in Sections 3.5-3.12 above.

Let $\alpha, \beta \in V\left(D^{2}, F_{n}\right)$ have the property that for every chord diagram $\Gamma$ and one (hence any) $c \in c(\Gamma),\langle\alpha \mid c\rangle= \pm\langle\beta \mid c\rangle$. We will show $\alpha= \pm \beta$, giving (v) $\Rightarrow$ $\{(\mathrm{i})$, (ii) $\}$ as desired. Write the decompositions of $\alpha$ and $\beta$ as $\alpha=\sum_{w \in \mathcal{W}_{n}^{e}} \alpha_{w} c_{w}$, $\beta=\sum_{w \in \mathcal{W}_{n}^{e}} \beta_{w} c_{w}$.

Using the isomorphism $V\left(D^{2}\right) \cong \mathcal{F}$, and the map $Q_{-}$(Section 2.6), we have $\langle\alpha \mid c\rangle=$ $\left(Q_{-}^{-1} \alpha\right) \cdot c$ and $\langle\beta \mid c\rangle=\left(Q_{-}^{-1} \beta\right) \cdot c$. Rewriting $Q_{-}^{-1} \alpha, Q_{-}^{-1} \beta$ as $\alpha, \beta$, we have two elements $\alpha, \beta \in V\left(D^{2}, F_{n}\right)$ such that $\alpha \cdot c= \pm \beta \cdot c$ for all suture elements $c$; it is sufficient to show $\alpha= \pm \beta$. Consider now $c$ to run through basis elements $c_{w}$, for $w \in \mathcal{W}_{n}^{e}$; we obtain, for every $w \in \mathcal{W}_{n}^{e}, \alpha_{w}= \pm \beta_{w}$.

Partition $\mathcal{W}_{n}^{e}$ into three subsets

$$
\begin{aligned}
& S=\left\{w \in \mathcal{W}_{n}^{e}: \alpha_{w}=\beta_{w}=0\right\}, \\
& T=\left\{w \in \mathcal{W}_{n}^{e}: 0 \neq \alpha_{w}=\beta_{w}\right\}, \\
& U=\left\{w \in \mathcal{W}_{n}^{e}: 0 \neq \alpha_{w}=-\beta_{w}\right\}
\end{aligned}
$$

We will show one of $T$ or $U$ is empty. Suppose not. Consider the set $A$ of suture elements $c \in V\left(D^{2}, F_{n}\right)$ whose basis decomposition contains words from both $T$ and $U$. As $T$ and $U$ are nonempty, Lemma 3.19 guarantees $A$ is nonempty. For $c \in A$, letting $\pm c= \pm\left[w_{0}, w_{1}\right]$, define $l(c)=d\left(w_{0}, w_{1}\right)$ (Definition 2.3). By definition $A$ contains no basis elements, so $l(c)>0$.

Take $c \in A$ with $l(c)$ minimal; let $\pm c= \pm\left[w_{0}, w_{1}\right]$, so $w_{0} \leq w_{1}, w_{0} \neq w_{1}$. We claim that in the basis decomposition of $c$ there is only one word from $T$ and one from $U$. (There may be many from $S$.) Suppose not, so without loss of generality $c$ contains distinct words $t_{0}, t_{1} \in T$ and $u \in U$.

Let $w_{-}=\min \left(t_{0}, u\right)$ and let $w_{+}=\max \left(t_{0}, u\right)$. By definition of $w_{0}$ we have $w_{0} \leq t_{0}, u$ hence $w_{0} \leq w_{-}$; indeed $w_{0} \leq w_{-} \leq w_{+} \leq w_{1}$. Thus $d\left(w_{-}, w_{+}\right) \leq d\left(w_{0}, w_{1}\right)$. By Lemma 3.19, $\pm\left[w_{-}, w_{+}\right]$contains $t_{0}, u$ with nonzero coefficients; so $\pm\left[w_{-}, w_{+}\right] \in A$ and $l\left( \pm\left[w_{-}, w_{+}\right]\right) \leq l\left( \pm\left[w_{0}, w_{1}\right]\right)=l(c)$. By minimality of $c$ then equality must hold; so $w_{0}=w_{-}$and $w_{+}=w_{1}$. In particular, $w_{-} \leq t_{1} \leq w_{+}$. As $w_{-}=\min \left(t_{0}, u\right)$ and $w_{+}=\max \left(t_{0}, u\right)$, then, for every $i \in\left\{1, \ldots, n_{x}\right\}$, the $i-$ th $x$ in $t_{1}$ lies in a position between the respective positions of the $i-$ th $x$ in $t_{0}$ and $u$ :

$$
\text { either } \quad h_{t_{0}}^{x}(i) \leq h_{t_{1}}^{x}(i) \leq h_{u}^{x}(i) \quad \text { or } \quad h_{u}^{x}(i) \leq h_{t_{1}}^{x}(i) \leq h_{t_{0}}^{x}(i) \text {. }
$$


But the same argument applies with $t_{0}$ and $t_{1}$ reversed; then for every $i \in\left\{1, \ldots, n_{x}\right\}$,

$$
\text { either } \quad h_{t_{1}}^{x}(i) \leq h_{t_{0}}^{x}(i) \leq h_{u}^{x}(i) \quad \text { or } \quad h_{u}^{x}(i) \leq h_{t_{0}}^{x}(i) \leq h_{t_{1}}^{x}(i) .
$$

Putting these together, we have that for all $i, h_{t_{0}}^{x}(i)=h_{t_{1}}^{x}(i)$, ie $t_{0}=t_{1}$, a contradiction.

Thus the suture element $c \in V\left(D^{2}, F_{n}\right)$ contains precisely one word $t$ from $T$ and one $u$ from $U$, with coefficients $\epsilon_{t}, \epsilon_{u} \in\{-1,1\}$; the rest must be in $S$. Then $\alpha \cdot c=\alpha_{t} \epsilon_{t}+\alpha_{u} \epsilon_{u}$ and $\beta \cdot c=\beta_{t} \epsilon_{t}+\beta_{u} \epsilon_{u}$. By definition of $T$ and $U, \alpha_{t}=\beta_{t}$ and $\alpha_{u}=-\beta_{u}$, and recall $\alpha \cdot c= \pm \beta \cdot c$. Hence

$$
\alpha_{t} \epsilon_{t}+\alpha_{u} \epsilon_{u}=\left\{\begin{array}{l}
\alpha_{t} \epsilon_{t}-\alpha_{u} \epsilon_{u}, \\
-\alpha_{t} \epsilon_{t}+\alpha_{u} \epsilon_{u} .
\end{array}\right.
$$

In the first case we have $2 \alpha_{u} \epsilon_{u}=0$ so $\alpha_{u}=0$; in the second $2 \alpha_{t} \epsilon_{t}=0$ so $\alpha_{t}=0$. These are both contradictions to the definitions of $T$ and $U$. Thus one of $T$ or $U$ is empty, and $\alpha= \pm \beta$.

Next we prove Proposition 3.8, using the idea of the "free presutured TQFT" (see Section 3.2).

Proof of Proposition 3.8 The equivalence of the two alternative axioms is immediate from Axiom 8, that each $V(\Sigma, F)$ is spanned by suture elements.

Define the sutured TQFT as follows: $V(\Sigma, F)=\bigoplus_{\Gamma} \mathbb{Z} c_{\Gamma}$ as for the free presutured TQFT; for any set of sutures $\Gamma$ with contractible components, $c(\Gamma)=\{0\}$; for $\Gamma$ without contractible components, $c(\Gamma)=\left\{c_{\Gamma},-c_{\Gamma}\right\}$. For a gluing $\tau$, gluing maps $\Phi_{\tau}^{i}$ must satisfy $c_{\Gamma} \mapsto \pm c_{\#_{\tau} \Gamma}$ or 0 as appropriate; we define many gluing maps, covering all sign possibilities. More precisely, each $\Phi_{\tau}^{i}: V(\Sigma, F) \longrightarrow V\left(\#_{\tau}(\Sigma, F)\right)$ takes $c_{\Gamma} \mapsto 0$, if $\#_{\tau} \Gamma$ has a contractible component; else takes $c_{\Gamma} \mapsto \pm c_{\#_{\tau} \Gamma}$, where the sign can be chosen freely. As the $c_{\Gamma}$ form a basis, a choice of signs defines $\Phi_{\tau}^{i}$. We allow all choices of signs, and these form our collection $\Phi_{\tau}^{i}$. It is clear that Axioms 1-8 are satisfied.

The bilinear map $\langle\cdot \mid \cdot\rangle: V\left(D^{2}, F_{n}\right) \otimes V\left(D^{2}, F_{n}\right) \longrightarrow \mathbb{Z}$ obtained from stacking has many sign choices; we will show that these sign choices can be made to satisfy alternative axiom (i), proving the result. For sutures $\Gamma_{0}, \Gamma_{1}$ on $\left(D^{2}, F_{n}\right),\left\langle c_{\Gamma_{0}} \mid c_{\Gamma_{1}}\right\rangle$ must be 0 , if $\Gamma_{0}, \Gamma_{1}$ are not stackable; otherwise $\left\langle c_{\Gamma_{0}} \mid c_{\Gamma_{1}}\right\rangle= \pm 1$, and we may choose either sign; as the $c_{\Gamma}$ form a basis, signs may be chosen independently.

Ordering arbitrarily, write $\Gamma_{1}, \Gamma_{2}, \ldots, \Gamma_{C_{n}}$ for the chord diagrams on $\left(D^{2}, F_{n}\right)$. Let $g_{i j}=\left\langle c_{\Gamma_{i}} \mid c_{\Gamma_{j}}\right\rangle$. For each pair $(i, j)$, either $g_{i j}$ is forced to be zero, or we can choose $g_{i j}= \pm 1$. The matrix $g=\left\{g_{i j}\right\}$ is the matrix for a bilinear map $\mathbb{Z}^{C_{n}} \otimes \mathbb{Z}^{C_{n}} \longrightarrow \mathbb{Z}$; 
or equally for a map $\mathbb{Q}^{C_{n}} \otimes \mathbb{Q}^{C_{n}} \longrightarrow \mathbb{Q}$. We see that (i) holds if the matrix $g$ over $\mathbb{Q}$ has full rank.

For $i \neq j$, choose $g_{i j}$ arbitrarily (if there is a choice!). Now for any chord diagram $\Gamma$, $\left\langle c_{\Gamma} \mid c_{\Gamma}\right\rangle= \pm 1$; each diagonal element $g_{i i}= \pm 1$. Consider row-reducing $g$. Successively choose the $g_{i i}$ so that, as row reduction proceeds to the $i$-th line, the $(i, i)$ element remains nonzero. After row reduction, we have an upper triangular matrix with all diagonal entries nonzero; hence it has full rank, and (i) is satisfied.

\subsection{An additional axiom}

We consider a tenth axiom for sutured TQFT. Note that as gluings can only increase genus, we could simply set $V(\Sigma, F)=0$ whenever $\Sigma$ has genus $\geq 1$, and obtain a sutured TQFT. Our additional axiom requires certain gluing maps to be isomorphisms.

Axiom 10 Let $\tau$ be a sutured gluing map on $(\Sigma, F)$, identifying two disjoint arcs $\gamma, \gamma^{\prime}$ on $\partial \Sigma$. If $|\gamma \cap F|=\left|\gamma^{\prime} \cap F\right|=1$, then any associated gluing map $\Phi_{\tau}^{i}$ is an isomorphism.

Any connected sutured background $(\Sigma, F)$ can be constructed from a $\left(D^{2}, F_{n}\right)$ by gluing maps of this type; in fact from a disjoint union of discs $\bigsqcup\left(D^{2}, F_{2}\right)$. So this final axiom gives isomorphisms $V(\Sigma, F) \cong V\left(\bigsqcup_{i}\left(D^{2}, F_{2}\right)\right) \cong \bigotimes_{i} V\left(D^{2}, F_{2}\right)$. Axiom 9 becomes redundant, and Axiom 8 almost redundant.

Lemma 3.20 In the presence of Axioms 1-8, Axiom 10 implies Axiom 9.

Proof On $\left(D^{2}, F_{2}\right)$ there are only two chord diagrams; as in Section 3.6, inclusions $\left(D^{2}, F_{2}\right) \hookrightarrow\left(D^{2}, F_{1}\right)$ with intermediate sutures can be found to show that their suture elements are linearly independent. Two such suture elements span $V\left(D^{2}, F_{2}\right)$; they form a basis which we denote $x, y$ and in fact $V\left(D^{2}, F_{2}\right)=V\left(D^{2}\right)_{1} \cong \mathcal{F}_{1}$ as graded abelian groups.

Gluing two $\left(D^{2}, F_{2}\right)$ 's gives an isomorphism $V\left(D^{2}, F_{3}\right)=V\left(D^{2}\right)_{2} \cong(\mathbb{Z} x \oplus \mathbb{Z} y)^{\otimes 2}=$ $\mathcal{F}_{2}$; the summand $V\left(D^{2}\right)_{2}^{0}$ has basis $\{x \otimes y, y \otimes x\}$, which are suture elements (exactly like $x y, y x$ above) for bypass-related chord diagrams. Including $\left(D^{2}, F_{3}\right) \hookrightarrow\left(D^{2}, F_{1}\right)$ in various ways, we obtain coordinate projections $\mathbb{Z}^{2} \cong V\left(D^{2}\right)_{2}^{0} \longrightarrow V\left(D^{2}, F_{1}\right) \cong \mathbb{Z}$; from these we see that the third chord diagram in the bypass triple is $\pm x \otimes y \pm y \otimes x$. By Proposition 3.7, this is equivalent to Axiom 9. 
Lemma 3.21 Assume Axioms 1-7. Suppose on $\left(D^{2}, F_{2}\right)$ the two chord diagrams, one each in Euler class -1 and 1 , have suture elements $\pm x, \pm y$ respectively where $\{x, y\}$ is a basis for $V\left(D^{2}, F_{2}\right)$. Then Axiom 10 implies Axiom 8. (And hence, by Lemma 3.20, also Axiom 9.)

Proof Since $x, y$ form a basis for $V\left(D^{2}, F_{2}\right)$, gluing $n\left(D^{2}, F_{2}\right)$ 's together, gluing isomorphisms (by Axiom 10) give a basis of suture elements $\{x, y\}^{\otimes n}$ for $V\left(D^{2}, F_{n+1}\right)$. Thus each $V\left(D^{2}, F_{n}\right)$ is spanned by suture elements, and by further gluing we obtain a basis of any $V(\Sigma, F)$ of suture elements.

\section{Sutured Floer homology and sutured TQFT}

We now show how sutured Floer homology [14] defines a sutured TQFT. As noted in [10], $\operatorname{SFH}\left(\Sigma \times S^{1}, F \times S^{1}\right)$, where $\Sigma$ is a surface with $\partial \Sigma \neq \varnothing$ and $F \subset \partial \Sigma$ is finite, has TQFT-like properties; in fact sutured TQFT is designed to be an axiomatic model for it. Make the following assignments.

- To a sutured background $(\Sigma, F)$, assign the abelian group $V(\Sigma, F)=$ $S F H\left(-\Sigma \times S^{1},-F \times S^{1}\right)$ with $\mathbb{Z}$ coefficients. It is known that SFH splits as a direct sum over spin-c structures.

- A set of sutures $\Gamma$ on $(\Sigma, F)$ without contractible components corresponds precisely to an isotopy class of tight contact structures $\xi$ on $\Sigma \times S^{1}$, such that $\partial \Sigma \times S^{1}$ is convex with dividing set $F \times S^{1}[7 ; 8 ; 9]$. Let $c(\Gamma)$ be the contact invariant $c(\xi) \subset V(\Sigma, F)[18 ; 12 ; 11]$. This $c(\xi)$ is a subset of the form $\{ \pm x\}$. The possible relative Euler classes of $\xi$ correspond to the spin-c structures on $(\Sigma, F)$; and $c(\xi)$ lies in the corresponding spin-c summand of SFH.

- For a gluing $\tau$ of the sutured background surface $(\Sigma, F)$, let

$$
\Phi_{\tau}: S F H\left(-\Sigma \times S^{1},-F \times S^{1}\right) \longrightarrow S F H\left(-\left(\#_{\tau} \Sigma\right) \times S^{1},-\left(\#_{\tau} F\right) \times S^{1}\right)
$$

be the map defined in [10] by the obvious inclusion $\Sigma \times S^{1} \hookrightarrow \#_{\tau} \Sigma \times S^{1}$, together with the canonical contact structure on $\#_{\tau} \Sigma \times S^{1}-\Sigma \times S^{1}$ as convex neighbourhood of the boundary. In fact we can choose a sign on each $\Phi_{\tau}$ on each Euler class summand; let $\Phi_{\tau}^{i}$ be the collection of all maps obtained from $\Phi_{\tau}$ by all possible choices of signs.

Proposition 4.1 These assignments satisfy all axioms of sutured TQFT (including Axiom 10). 
Proof Clearly Axioms 1-3 are satisfied. Axiom 4, $V\left(\bigsqcup_{i}\left(\Sigma_{i}, F_{i}\right)\right)=\bigotimes_{i} V\left(\Sigma_{i}, F_{i}\right)$, is clear from the definition of SFH. Axiom 5, that a gluing map takes contact elements to contact elements, is proved in [10]. If $\Gamma$ contains a contractible loop then the corresponding contact structure is overtwisted; by [11] then $c(\Gamma)=\{0\}$, so Axiom 6 holds. That $V\left(D^{2}, F_{1}\right) \cong \mathbb{Z}$ is proved in [10] and follows from [15] or [11]; that $c\left(\Gamma_{\varnothing}\right)=\{ \pm 1\}$ is proved in [10] and follows from [11]; so Axiom 7 holds.

Axiom 10 is proved in [10]. Moreover, [10] shows, following [11], that $V\left(D^{2}, F_{2}\right) \cong \mathbb{Z}^{2}$, with basis given by contact elements for the two chord diagrams of Euler class -1 and 1. By Lemma 3.21, this implies Axiom 8; then Lemma 3.20 gives Axiom 9.

Thus, results about sutured TQFT gives results about SFH. In particular, contact elements in the SFH of sutured solid tori with longitudinal sutures have all the structure of $V\left(D^{2}\right)$.

\section{Corollary 4.2 A sutured TQFT exists.}

Corollary 4.2 could of course also be proved by explicit construction. On discs we could use all the machinery of the foregoing; however on more complicated surfaces, a nonzero construction would take more work.

\section{Noncommutative QFT = sutured TQFT of discs}

\subsection{Main isomorphism and suture elements}

We now make a detailed statement that "the sutured TQFT of discs is the QFT of two noncommuting particles"; most of this is already clear. This includes earlier Theorems 1.1 and 3.4.

Theorem 5.1 Every sutured TQFT obeying Axioms 1-9 above satisfies

$$
V\left(D^{2}\right) \cong \mathcal{F},
$$

an isomorphism of bigraded rings. In particular

$$
V\left(D^{2}\right)_{n+1} \cong \mathcal{F}_{n} \cong \mathbb{Z}^{2^{n}}, \quad V\left(D^{2}\right)_{n_{-}, n_{+}} \cong \mathcal{F}_{n_{x}, n_{y}} \cong \mathbb{Z}^{\left(\begin{array}{c}
n \\
n_{x}
\end{array}\right)} \cong \mathbb{Z}^{\left(\begin{array}{c}
n \\
n_{y}
\end{array}\right)},
$$

isomorphisms of abelian groups. Each $c_{w} \in V\left(D^{2}\right)_{n}^{e}$, for $w \in \mathcal{W}_{n}^{e}$, corresponds to $w \in \mathcal{M}_{n}^{e} \subset \mathcal{F}_{n}^{e}$, replacing $(-,+)$ with $(x, y)$. Under this isomorphism:

(i) The action of operators $a_{x, i}, a_{y, i}, a_{x, i}^{*}, a_{y, i}^{*}$ on words $w \in \mathcal{M}_{n}^{e} \subset \mathcal{F}_{n}^{e}$ is identical to the action of operators $a_{-, i}, a_{+, i}, a_{-, i}^{*}, a_{+, i}^{*}$ on basis suture elements $c_{w}$, for $w \in \mathcal{W}_{n}^{e}$. 
(ii) The $\langle\cdot \mid \cdot\rangle$ defined on $\mathcal{F}$ via the partial order $\leq$, and the $\langle\cdot \mid \cdot\rangle$ defined on $V\left(D^{2}\right)$ by stacking, agree.

(iii) The operators $T_{x, i}, T_{y, i}, T_{x, i}^{*}, T_{y, i}^{*}, U_{x, i}, U_{y, i}$ act on each $\mathcal{F}_{n}^{e}$ identically to the operators $T_{-, i}, T_{+, i}, T_{-, i}^{*}, T_{+, i}^{*}, U_{-, i}, U_{+, i}$ on $V\left(D^{2}\right)_{n}^{e}$.

(iv) The duality operator $H$ acts on $\mathcal{F}_{n}^{e}$ identically to the rotation operator $R$ on $V\left(D^{2}\right)_{n}^{e}$.

(v) The set of suture elements in $V\left(D^{2}\right)_{n}^{e}$ maps to the distinguished subset $\mathcal{C}_{n}^{e}$.

Most of this theorem has already been proved. The isomorphism of rings, $\langle\cdot \mid \cdot\rangle, a^{*}$, $a, T, T^{*}, U, H$ and $R$ is clear (indeed signs were chosen carefully to ensure it). It only remains to prove the last statement $(\mathrm{v})$. But to do this we need to establish that $\mathcal{C}_{n}^{e}$ exists, in particular Proposition 2.9 that the three definitions $\mathcal{C}^{1}, \mathcal{C}^{2}, \mathcal{C}^{3}$ all agree. We prove Proposition 2.9 and Theorem 5.1 together by showing that all of $\mathcal{C}^{1}, \mathcal{C}^{2}, \mathcal{C}^{3}$ map to the set of suture elements.

Note that our proof of Proposition 2.9 relies upon an isomorphism to sutured TQFT, and hence upon the existence of a sutured TQFT; thus, at least in our presentation, there is a dependence upon sutured Floer homology.

Proof of Proposition 2.9 and Theorem 5.1(v) We first show $\mathcal{C}^{1}$ corresponds to suture elements. Clearly suture elements are preserved under $a_{s, i}, a_{s, i}^{*}$, and $T_{ \pm, i}^{*}$; we show every suture element can be created from the vacuum by these operators. Clearly the 0 suture element can be obtained. For any chord diagram $\Gamma$ with $n+1$ chords, we produce an element of $c(\Gamma)$.

Proof by induction on $n$. Clearly we can obtain the vacuum. If $\Gamma$ has an outermost chord at the basepoint, or at the root point, or has an outermost chord enclosing a positive region on the eastside, or enclosing a negative region on the westside, then $\Gamma$ is obtained from a smaller chord diagram by applying a creation operator, and we reduce to a smaller diagram. So we may assume all outermost regions are negative and on the eastside, or positive and on the westside.

Suppose there is an outermost negative region on the eastside; the other case is similar. Then there is one closest to the basepoint, enclosed by a chord running from $2 i+1$ to $2 i+2$, where $i \geq 0$ is minimal. (Labelling as in Section 3.1.) Then (Figure 12) $\Gamma$ is obtained from a chord diagram $\Gamma^{\prime}$ by applying $a_{+, i+2} T_{+, i}^{*}$, where $\Gamma^{\prime}$ is identical to $\Gamma$, except that the outermost chord at $(2 i+1,2 i+2)$ is moved to $(2 i-1,2 i)$. The outermost negative region moves closer to the basepoint; repeating, we eventually have an outermost chord at the basepoint, and can reduce to a smaller diagram. 


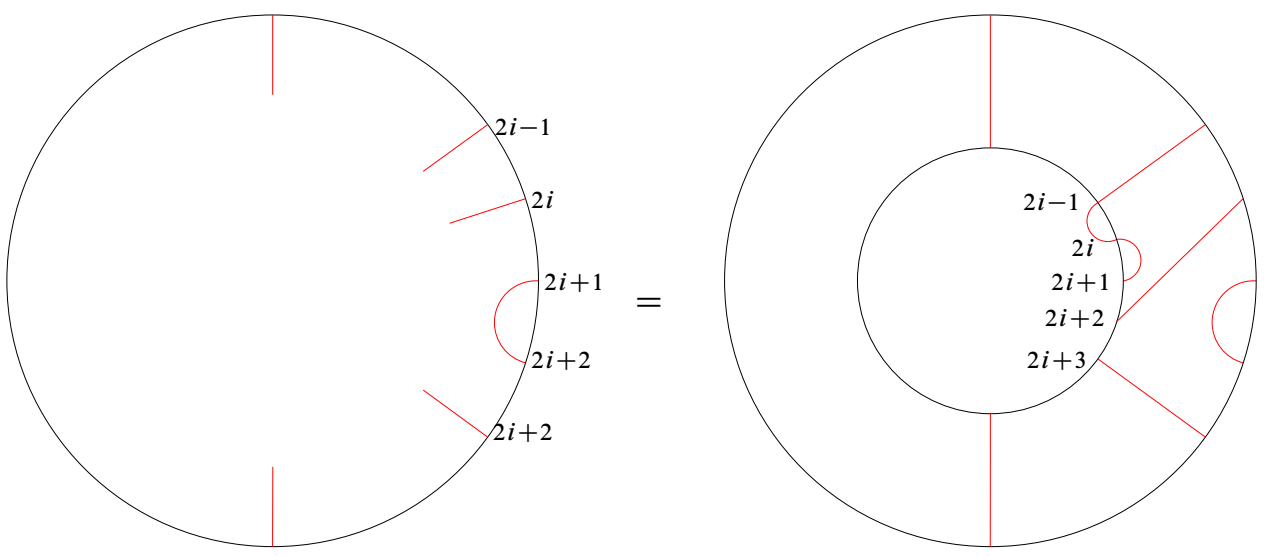

Figure 12: A chord diagram with outermost region at $(2 i+1,2 i+2)$ is $a_{+, i+2} T_{+, i}^{*}$ of an otherwise identical chord diagram with outermost region at $(2 i-1,2 i)$.

Thus, for any chord diagram $\Gamma$, we can obtain a suture element in $c(\Gamma)$. To obtain both, it suffices to show we can obtain -1 , which we do as follows. Hence $\mathcal{C}^{1}$ coincides with suture elements.

$$
1 \stackrel{a_{x, 0}^{*}}{\longmapsto} x \stackrel{T_{y, 0}^{*}}{\longmapsto} y x-x y \stackrel{a_{x, 0}}{\longmapsto}-y \stackrel{a_{y, 0}}{\longmapsto}-1 .
$$

For $\mathcal{C}^{2}$, suture elements are clearly preserved by $a_{s, 0}^{*}$ and $H$. We can clearly obtain 0 . For a chord diagram $\Gamma$ of $n+1$ chords, we show we can produce an element of $c(\Gamma)$; again, induct on number of chords. The vacuum is clear. Now any $\Gamma$ has an outermost chord; applying $H$ this may be rotated to the basepoint; this diagram may be obtained from a smaller one via $a_{s, 0}^{*}$. To show we can obtain -1 , we need $H^{3}(x y)=-x y$ on $\mathcal{F}_{1,1}$, which can be computed by hand from Corollary 2.8 (we prove Theorem 2.6, the general result, in Section 5.4). So $\mathcal{C}^{2}$ coincides with suture elements.

$$
1 \stackrel{a_{y, 0}^{*}}{\longmapsto} y \stackrel{a_{x, 0}^{*}}{\longmapsto} x y \stackrel{H^{3}}{\longmapsto}-x y \stackrel{a_{x, 0}}{\longmapsto}-y \stackrel{a_{y, 0}}{\longmapsto}-1 .
$$

The proof for $\mathcal{C}^{3}$ is similar, replacing basepoint with root point.

We now prove the algebraic statements about suture elements of Theorem 2.10. (Again, this proof relies on sutured TQFT existence.)

Proof of Theorem 2.10 Part (i) is now clear; (ii) is a statement of properties of suture elements, as discussed in Sections 3.7 and 3.9. For (iii), it's easy to check $Q_{ \pm}$does not preserve $\mathcal{C}$; but it is a bijection (we have computed an inverse), hence 
$\left|Q_{ \pm} \mathcal{C}_{n}^{e}\right|=\left|\mathcal{C}_{n}^{e}\right|=2 N_{n}^{e}$; and since $Q_{ \pm}{ }^{\circ} Q_{\mp}^{-1}=H^{ \pm 1}$ preserves $\mathcal{C}$ we have $Q_{+} \mathcal{C}=Q_{-} \mathcal{C}$. Part (iv) follows from Proposition 3.16, definition of $H$ and $\langle\cdot \mid \cdot\rangle$.

For (v), if the sum/difference of suture elements $u \in c\left(\Gamma_{u}\right), v \in c\left(\Gamma_{v}\right)$ is also a suture element, then by [17, Proposition 1.10], $\Gamma_{u}, \Gamma_{v}$ are bypass-related. After possibly reordering and switching signs, $\langle u \mid v\rangle=1,\langle v \mid u\rangle=0$. Then $u-v$ is a suture element for the third diagram. Being bypass-related, $\Gamma_{u}, \Gamma_{v}$ may be isotoped to be identical, except in a disc $D^{\prime} \subset D$; and we may choose a basepoint on $D^{\prime}$ such that $\Gamma_{u} \cap D^{\prime}=\Gamma_{x y}$, $\Gamma_{v} \cap D^{\prime}=\Gamma_{y x}$; these have suture elements $x y, y x$ respectively, and $\langle x y \mid y x\rangle=1$. Now $\Gamma_{u}, \Gamma_{v}$ can be obtained by applying initial creation operators and rotations to $\Gamma_{x y}, \Gamma_{y x}$, so we obtain an operator $A^{*}$ taking $x y \mapsto \pm u, y x \mapsto \pm v$. Since creations and rotations are isometries, we have $1=\langle x y \mid y x\rangle=\left\langle A^{*}(x y) \mid A^{*}(y x)\right\rangle=\langle u \mid v\rangle$; thus under $A^{*},(x y, y x) \mapsto \pm(u, v)$. If we get $-(u, v)$, then precompose $A^{*}$ with $H^{3}=-1$ on $\mathcal{F}_{1,1}$.

Part (vi) is essentially [17, Lemma 3.1] without contact geometry and with signs. Given chord diagrams $\Gamma_{u}, \Gamma_{v}$ with $u \in c\left(\Gamma_{u}\right), v \in c\left(\Gamma_{v}\right)$ and $\langle u \mid v\rangle=1$, we find chord diagrams $\Gamma_{u}=\Gamma_{0}, \Gamma_{1}, \ldots, \Gamma_{m}=\Gamma_{v}$, and $u=c_{0}, c_{1}, \ldots, c_{m}=v$ where $c_{i} \in c\left(\Gamma_{i}\right)$, such that each $c_{i}-c_{i+1}$ is a suture element - in particular, each pair $\Gamma_{i}, \Gamma_{i+1}$ is bypass-related - and for all $i \leq j,\left\langle c_{i} \mid c_{j}\right\rangle=1$. Proof by induction on the number of chords in $\Gamma_{u}, \Gamma_{v}$. With less than three chords there is nothing to prove; with three chords $\Gamma_{u}, \Gamma_{v}$ are either identical or bypass-related, and the result is clear.

For general $\Gamma_{u}, \Gamma_{v}$, if they share an outermost chord $\gamma$ then consider $\Gamma_{u}-\gamma$ and $\Gamma_{u}-\gamma$; by induction we have a sequence of bypass-related chord diagrams with the desired properties; adding $\gamma$ to all these gives an isometry (Section 3.12). Hence we obtain desired chord diagrams and suture elements.

Thus we may assume $\Gamma_{u}, \Gamma_{v}$ have no outermost chords in common. Let $\gamma$ be an outermost chord of $\Gamma_{v}$; denote its endpoints $p, q$ in clockwise order, and the next marked point clockwise $r$. On $\Gamma_{u}$ there is no outermost chord connecting $p, q$ (by assumption), nor connecting $q, r$ (since then rounding would give $\langle u \mid v\rangle=0$ ). Thus $\Gamma_{u}, \Gamma_{v}$ appear as in Figure 13. Perform upwards bypass surgery on $\Gamma_{u}=\Gamma_{0}$ along the arc $\delta$ shown to define $\Gamma_{1}$.

Now, $\Gamma_{1}, \Gamma_{v}$ share the common outermost chord $\gamma$; from above, we obtain a sequence of chord diagrams $\Gamma_{1}, \Gamma_{2}, \ldots, \Gamma_{m}$, where each $\Gamma_{i}, \Gamma_{i+1}$ are bypass-related, and suture elements $c_{1}, \ldots, c_{m}$, where $c_{i} \in c\left(\Gamma_{i}\right)$ satisfy $\left\langle c_{i} \mid c_{j}\right\rangle=1$ for $1 \leq i \leq j \leq m$ and each $c_{i}-c_{i+1}$ is a suture element. Adjusting the $c_{i}$ by a sign if necessary, we may assume $c_{m}=v$. Taking $c_{0}=u$ and $\Gamma_{0}=\Gamma_{u}$, we claim that the $\Gamma_{i}$ and $c_{i}$ have the desired properties; we must verify that $c_{0}-c_{1}$ is a suture element and that $\left\langle c_{0} \mid c_{i}\right\rangle=1$ for $0 \leq i \leq m$. 


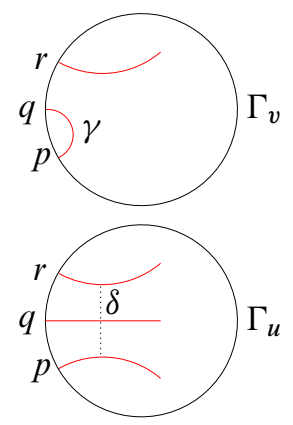

Figure 13: Chord diagrams $\Gamma_{u}, \Gamma_{v}$

To see $c_{0}-c_{1}$ is a suture element, note that as $\Gamma_{0}, \Gamma_{1}$ are bypass-related, either $c_{0}-c_{1}$ or $c_{0}+c_{1}$ is a suture element; since $\left\langle c_{0} \mid c_{m}\right\rangle=\langle u \mid v\rangle=1$ and $\left\langle c_{1} \mid c_{m}\right\rangle=1$, we have $\left\langle c_{0} \pm c_{1} \mid c_{m}\right\rangle=2$ or 0 ; thus $c_{0}-c_{1}$ is a suture element.

We next show that for $1 \leq i \leq m,\left\langle c_{0} \mid c_{i}\right\rangle= \pm 1$. Since $\Gamma_{1}$ is obtained from $\Gamma_{0}$ by upwards bypass surgery, $\left\langle c_{0} \mid c_{1}\right\rangle= \pm 1$. Now for $2 \leq i \leq m$, consider the sutured cylinders which are rounded in computing $\left\langle c_{0} \mid c_{i}\right\rangle$ and $\left\langle c_{1} \mid c_{i}\right\rangle$. Note $\Gamma_{i}$ contains the outermost chord $\gamma$, which can be pushed down by a "finger move" to $\Gamma_{0}$ or $\Gamma_{1}$ to give identical sets of sutures. Since $\left\langle c_{1} \mid c_{i}\right\rangle=1$ then $\left\langle c_{0} \mid c_{i}\right\rangle= \pm 1$.

Finally we verify that for $0 \leq i \leq m,\left\langle c_{0} \mid c_{i}\right\rangle=1$. Obviously $\left\langle c_{0} \mid c_{0}\right\rangle=1$. As $c_{0}-c_{1}$ is a suture element, $\left\langle c_{0} \mid c_{i}\right\rangle= \pm 1$ and $\left\langle c_{1} \mid c_{i}\right\rangle=1$, we have $\left\langle c_{0}-c_{1} \mid c_{i}\right\rangle= \pm 1-1=0$ or -2 . Hence $\left\langle c_{0} \mid c_{i}\right\rangle=1$.

The proof of (vi) here effectively gives another proof of [17, Lemma 3.1]. The proof constructs a tight contact structure on a solid cylinder by bypass attachments.

\subsection{Duality-rotation explicitly}

We now prove some detailed results about the $H=R$ duality/rotation operator. In [17] we obtained $(\bmod 2)$ recursive and explicit descriptions of $H$; we prove these here over $\mathbb{Z}$.

Recall the formula of Corollary 2.8, including the exceptional sets $E_{w}^{s}$ and the operators $\psi_{T}^{s}$ :

$$
H w=Q_{+} Q_{-}^{-1} w=\sum_{w_{i} \geq w} \sum_{T \subseteq E_{w_{i}}^{y}}(-1)^{|T|} \psi_{T}^{y} w_{i}
$$

Regard $x$ 's as pawns and $y$ 's as empty squares, so $\psi_{T}^{x} w$ moves some pawns forward (right) one square each; $w \leq \psi_{T}^{x} w$. Call any $\psi_{T}^{x} w$ pawn-ahead of $w$. Similarly, 
any $\psi_{T}^{y} w \leq w$; call any $\psi_{T}^{y} w$ pawn-behind $w$. Recall $f_{w}^{x}(i)$ is the number of $y$ 's to the left of the $i-$ th $x$ in $w$.

Proposition 5.2 Let $w \in \mathcal{M}_{n}^{e}$. The words $v$ occurring in $H w$ are precisely those such that:

(i) If $i \in E_{v}^{x}$ then $f_{v}^{x}(i)=f_{w}^{x}(i)-1$.

(ii) If $i \notin E_{v}^{x}$ then $f_{v}^{x}(i) \geq f_{w}^{x}$.

The coefficient of $v$ in $H w$ is $(-1)^{\left|E_{v}^{x}\right|}$.

Proof From the formula, we have immediately that any $v$ occurring in $H w$ is pawnbehind some $u$ with $w \leq u$. So for $v$ occurring in $H w, f_{v}^{x}(i) \geq f_{w}^{x}(i)$ for $i \notin E_{v}^{x}$, and $f_{v}^{x}(i) \geq f_{w}^{x}(i)-1$ for $i \in E_{v}^{x}$. Partition $\left\{1,2, \ldots, n_{x}\right\}$ into three sets

$$
\begin{aligned}
& S_{0}=\left\{1,2, \ldots, n_{x}\right\}-E_{v}^{x}, \\
& S_{1}=\left\{i \in E_{v}^{x}: f_{v}^{x}(i)=f_{w}^{x}(i)-1\right\}, \\
& S_{2}=\left\{i \in E_{v}^{x}: f_{v}^{x}(i) \geq f_{w}^{x}(i)\right\} .
\end{aligned}
$$

In fact, $v$ appears once for every $u \geq w$ pawn-ahead of $v$. Such $u$ are precisely those obtained by moving up all the $x$-pawns in $S_{1}$, and some subset $A$ of the $x$-pawns in $S_{2}$. Thus, terms in the sum for $H w$ involving $v$ are precisely

$$
\sum_{A \subseteq S_{2}}(-1)^{\left|A \cup S_{1}\right|} v=(-1)^{\left|S_{1}\right|} v \sum_{A \subseteq S_{2}}(-1)^{|A|} .
$$

If $S_{2} \neq \varnothing$ this sum is 0 ; if $S_{2}=\varnothing$ then $v$ appears in $H w$ with coefficient $(-1)^{\left|S_{1}\right|}$.

So, the $v$ occurring in $H w$ are precisely those with $f_{v}^{x}(i) \geq f_{w}^{x}(i)$ for $i \notin E_{v}^{x}$, and such that $S_{2}=\varnothing$, ie $f_{v}^{x}(i)=f_{w}^{x}(i)-1$ for all $i \in E_{v}^{x}$. The coefficient of such a $v$ is $(-1)^{\left|S_{1}\right|}$, and $S_{1}=E_{v}^{x}$.

In general, take a word

$$
w=x^{a_{1}} y^{b_{1}} x^{a_{2}} y^{b_{2}} \ldots x^{a_{k}} y^{b_{k}}
$$

where $k \geq 2$, possibly $a_{1}=0$, possibly $b_{k}=0$, but all other $a_{i}, b_{i} \neq 0$. Consider $v$ satisfying the conditions of the above proposition

$$
v=y^{\beta_{1}} x^{\alpha_{1}} \ldots y^{\beta_{l}} x^{\alpha_{l}}
$$


for some positive integer $l$; possibly $\beta_{1}=0$, possibly $\alpha_{l}=0$ but all other $\alpha_{i}, \beta_{i} \neq 0$. The values of $f_{v}^{x}$ are given by the cumulative sums $\beta_{1}+\cdots+\beta_{i}$, and the values of $f_{w}^{x}$ by cumulative sums $b_{1}+\cdots+b_{j}$. By condition (i) of Proposition 5.2 we must have

$$
\beta_{1}+\cdots+\beta_{i}=b_{1}+\cdots+b_{P(i)}-1
$$

for $1 \leq i \leq l-1$, for some increasing sequence $1 \leq P(1)<P(2)<\cdots<P(l-1) \leq k$. And of course $\beta_{1}+\cdots+\beta_{l}=b_{1}+\cdots+b_{k}$, so set $P(l)=k$. To maintain the two conditions of the proposition, we must also have the $x$ 's ending each block in $v$ corresponding to the $x$ beginning the next block in $w$; thus

$$
\alpha_{1}+\cdots+\alpha_{i}=a_{1}+\cdots+a_{P(i)}+1
$$

This term appears with sign $(-1)^{l-1}$. So $H w$ is the sum of the terms

$$
\begin{gathered}
(-1)^{l-1} y^{b_{1}+\cdots+b_{P(1)}-1} x^{a_{1}+\cdots+a_{P(1)}+1} y^{b_{P(1)+1}+\cdots+b_{P(2)}} x^{a_{P(1)+1}+\cdots+a_{P(2)}} \cdots \\
\cdots y^{b_{P(l-2)+1}+\cdots+b_{P(l-1)}} x^{a_{P(l-2)+1}+\cdots+a_{P(l-1)}} \\
\cdot y^{b_{P(l-1)+1}+\cdots+b_{P(l)}+1} x^{a_{P(l-1)+1}+\cdots+a_{P(l)}-1}
\end{gathered}
$$

over all $l \leq k$ and all increasing sequences $1 \leq P(1)<P(2)<\cdots<P(l)=k$ (with the exception that for the trivial sequence $l=1, P(1)=k$ the term is $y^{n_{y}} x^{n_{x}}$ ). There are $2^{k-1}$ terms in the sum. Recall here $k \geq 2$; if $k=1$ then we can easily check $H x^{n}=x^{n}, H y^{n}=y^{n}, H x^{n_{x}} y^{n_{y}}=y^{n_{y}} x^{n_{x}}$. This gives [17, Proposition 7.2], now with signs.

\subsection{Duality-rotation recursively}

We prove [17, Proposition 7.1], now with signs. Note the change of notation: $A_{-}, B_{-}$, $A_{+}, B_{+}$of [17] are now respectively $a_{y, 0}, a_{x, 0}^{*}, a_{x, 0}, a_{y, 0}^{*}$.

\section{Lemma 5.3}

(i) $a_{y, 0} H a_{y, 0}^{*}=H$.

(ii) $a_{y, 0} H\left(a_{x, 0}^{*}\right)^{j} a_{y, 0}^{*}=H\left(a_{x, 0}^{*}\right)^{j}$.

(iii) $a_{y, 0}\left(a_{x, 0}\right)^{j+1} H\left(a_{x, 0}^{*}\right)^{j} a_{y, 0}^{*} a_{x, 0}^{*}=-H$.

(iv) (a) $a_{y, 0}\left(a_{x, 0}\right)^{j} H\left(a_{x, 0}\right)^{j}=0$ for $j=1, \ldots, n_{x}$.

(b) $\left(a_{x, 0}\right)^{j+2} H\left(a_{x, 0}^{*}\right)^{j} a_{y, 0}^{*}=0$.

(c) $a_{x, 0} H\left(a_{x, 0}^{*}\right)^{j}\left(a_{y, 0}^{*}\right)^{2}=0$. 
Proof Since all creations and annihilations are initial, we drop 0 from the notation. For (i), we have

$$
\left\langle v \mid a_{y} H a_{y}^{*} w\right\rangle=\left\langle a_{y}^{*} v \mid H a_{y}^{*} w\right\rangle=\left\langle a_{y}^{*} w \mid a_{y}^{*} v\right\rangle=\langle w \mid v\rangle=\langle v \mid H w\rangle .
$$

Here we use the adjoint property; then definition of $H$; then creation operators are isometries; then definition of $H$. For (ii),

$$
\begin{aligned}
\left\langle v \mid a_{y} H\left(a_{x}^{*}\right)^{j} a_{y}^{*} w\right\rangle=\left\langle a_{y}^{*} v \mid H\left(a_{x}^{*}\right)^{j} a_{y}^{*} w\right\rangle & =\left\langle\left(a_{x}^{*}\right)^{j} a_{y}^{*} w \mid a_{y}^{*} v\right\rangle \\
& =\left\langle\left(a_{x}^{*}\right)^{j} w \mid v\right\rangle=\left\langle v \mid H\left(a_{x}^{*}\right)^{j} w\right\rangle .
\end{aligned}
$$

First we use the adjoint property; second and fourth the definition of $H$; the third equality follows from the observation that $x^{j} y w \leq y v$ if and only if $x^{j} w \leq v$.

Identity (iii) is the most difficult; first observe that in

$$
\left\langle v \mid a_{y}\left(a_{x}\right)^{j+1} H\left(a_{x}^{*}\right)^{j} a_{y}^{*} a_{x}^{*} w\right\rangle,
$$

any term of $H\left(a_{x}^{*}\right)^{j} a_{y}^{*} a_{x}^{*} w$ which does not begin with $x^{j+1} y$ is annihilated (to 0 !) by the operators $a_{y}\left(a_{x}\right)^{j+1}$; for each term $x^{j+1} y u$ occurring, the above inner product outputs $\langle v \mid u\rangle$.

Now note that if $v \leq u$ then $x^{j+1} y v \leq x^{j+1} y u$, and $x^{j} y x v \not \leq x^{j+1} y u$. But, for any word $z$ whose first $j+2$ symbols are not $x^{j+1} y$, we have $x^{j+1} y v \leq z$ if and only if $x^{j} y x v \leq z$. Thus

$$
\left\langle x^{j+1} y v-x^{j} y x v \mid z\right\rangle=\left\{\begin{array}{ll}
\langle v \mid u\rangle & \text { if } z=x^{j+1} y u, \\
0 & \text { otherwise }
\end{array}\right\}=\left\langle v \mid a_{y}\left(a_{x}\right)^{j+1} z\right\rangle .
$$

Hence

$$
\begin{aligned}
\langle v| a_{y}\left(a_{x}\right)^{j+1} H & \left.\left(a_{x}^{*}\right)^{j} a_{y}^{*} a_{x}^{*} w\right\rangle \\
& =\left\langle\left(a_{x}^{*}\right)^{j+1} a_{y}^{*} v \mid H\left(a_{x}^{*}\right)^{j} a_{y}^{*} a_{x}^{*} w\right\rangle-\left\langle\left(a_{x}^{*}\right)^{j} a_{y}^{*} a_{x}^{*} v \mid H\left(a_{x}^{*}\right)^{j} a_{y}^{*} a_{x}^{*} w\right\rangle \\
& =\left\langle\left(a_{x}^{*}\right)^{j} a_{y}^{*} a_{x}^{*} w \mid\left(a_{x}^{*}\right)^{j+1} a_{y}^{*} v\right\rangle-\left\langle\left(a_{x}^{*}\right)^{j} a_{y}^{*} a_{x}^{*} w \mid\left(a_{x}^{*}\right)^{j} a_{y}^{*} a_{x}^{*} v\right\rangle \\
& =-\langle w \mid v\rangle=-\langle v \mid H w\rangle,
\end{aligned}
$$

where we first use the previous observation, then the definition of $H$, then notice $x^{j} y x \not \leq x^{j+1} y$ and that creations are isometries, and finally the definition of $H$ again. This proves (iii).

We do not need to prove (iv) algebraically. As in [17], it is sufficient to observe that the operators on chord diagrams produce closed loops, so are 0 . 
Consider the matrix of each $H_{n_{x}, n_{y}}$. Following [17], for words $w_{0}, w_{1}$ (with $\leq n_{x} x$ 's and $\leq n_{y} \quad y$ 's), define the $w_{0} \times w_{1}$ minor of this matrix to be the intersection of the rows corresponding to words beginning with $w_{0}$, with the columns corresponding to words beginning with $w_{1}$. The above lemma gives the following description of $H_{n_{x}, n_{y}}$ :

(i) The $y \times y$ minor consists of $H_{n_{x}, n_{y}-1}$.

(ii) The $y \times x y$ minor contains the $x$-columns of $H_{n_{x}, n_{y}-1}$. More generally, for any $j=1, \ldots, n_{x}$, the $y \times x^{j} y$ minor contains the $x^{j}$-columns of $H_{n_{x}, n_{y}-1}$.

(iii) The $x y \times y x$ minor consists of $-H_{n_{x}-1, n_{y}-1}$. More generally, for any $j=$ $0, \ldots, n_{x}-1$, the $x^{j+1} y \times x^{j} y x$ minor consists of $-H_{n_{x}-j-1, n_{y}-1}$.

(iv) All other entries are zero.

We may write this recursive structure as a formula, as in [17]: the "fake" commutator (commutators do not mean much mod 2!) there becomes a real commutator here.

\section{Theorem 5.4}

$$
\begin{aligned}
H & =\sum_{i=0}^{\infty} a_{y, 0}^{*} H\left(a_{x, 0}^{*}\right)^{i} a_{y, 0}\left(a_{x, 0}\right)^{i}-\left(a_{x, 0}^{*}\right)^{i+1} a_{y, 0}^{*} H a_{x, 0} a_{y, 0}\left(a_{x, 0}\right)^{i} \\
& =\sum_{i=0}^{\infty}\left[a_{y, 0}^{*} H a_{x, 0},\left(a_{x, 0}^{*}\right)^{i+1}\right] a_{y, 0}\left(a_{x, 0}\right)^{i} .
\end{aligned}
$$

\subsection{Periodicity}

We now prove Theorem 2.6, $H^{n+1}=(-1)^{n_{x} n_{y}}$.

Consider $w \in \mathcal{M}_{n_{x}, n_{y}}$ as an $n \times 1$ chessboard; $x$ 's are pawns and $y$ 's are empty squares. Form a sequence of words / chessboard configurations, which begins at $w_{1}$ with all pawns / $x$ 's at the extreme right, $w_{1}=y^{n_{y}} x^{n_{x}}$. Pawns move one square left, if the square to their left is empty. Starting from $w_{1}$, form a sequence $w_{i}$ : on each turn all possible pawns move; we eventually arrive at $w_{n}$ with all pawns at the extreme left, $w_{n}=x^{n_{x}} y^{n_{y}}$. For example, with $n_{x}=2$ and $n_{y}=3$,

$$
w_{1}=\operatorname{yyyxx}, \quad w_{2}=y y x y x, \quad w_{3}=y x y x y, \quad w_{4}=x y x y y, \quad w_{5}=x x y y y .
$$

Obviously $w_{1}>w_{2}>\cdots>w_{n}$. We will compute $H\left[w_{i}, w_{i-1}\right]$.

As above, $\pm[u, v]$ denotes the suture elements with first word $u$ and last word $v$. Adopt the notation that $[u, v]$ is the suture element in which $u$ has coefficient 1 . Consider a 
suture element $\left[w_{i}, w_{i-1}\right], 2 \leq i \leq n$; it must take one of the following four forms:

$$
\begin{aligned}
{\left[w_{i}, w_{i-1}\right]=} & x^{\alpha}(x y-y x)^{\beta} x^{\gamma}, x^{\alpha}(x y-y x)^{\beta} y^{\gamma}, \\
& y^{\alpha}(x y-y x)^{\beta} x^{\gamma} \text { or } y^{\alpha}(x y-y x)^{\beta} y^{\gamma} .
\end{aligned}
$$

To see why, note that all the above expressions are products of suture elements, hence suture elements; expanding them out we obtain a sum where the lexicographically first is some $w_{i}$, and the last is some $w_{i-1}$; in fact any $w_{i}$ and $w_{i-1}$ can be obtained in this way.

Examining the explicit formula for $R=H$, we see that in any word $w$ beginning $w=x^{\alpha}(x y)^{k}(y x) \cdots$ or $y^{\alpha}(x y)^{k}(y x) \cdots$, where $k \geq 0$, the minimum word occurring in $H w$ begins

$$
\begin{aligned}
& \min H\left(x^{\alpha}(x y)^{k}(y x) \cdots\right)= \begin{cases}x^{\alpha+1}(x y)^{k} y x \cdots & k \geq 2, \\
y x^{\alpha+2} \cdots & k=1, \\
x^{\alpha+1} y \cdots & k=0,\end{cases} \\
& \min H\left(y^{\alpha}(x y)^{k}(y x) \cdots\right)= \begin{cases}y^{\alpha-1}(x y)^{k} y x \cdots & k \geq 1, \\
y^{\alpha} x \cdots & k=0 .\end{cases}
\end{aligned}
$$

On the other hand, in $H w_{i}$, the (lexicographically) first word occurring is $w_{i+1}$, with coefficient $(-1)^{\left|E_{w_{i+1}}^{x}\right|}$. Thus, for any $w \neq w_{i}$ occurring in $\left[w_{i}, w_{i-1}\right]$, the first word occurring in $H w$ is lexicographically after $w_{i+1}$. Hence for $2 \leq i \leq n-1$, $H\left(\left[w_{i}, w_{i-1}\right]\right)=(-1)^{\left|E_{w_{i+1}}^{x}\right|}\left[w_{i+1}, v\right]$ for some $v$.

We can see what this $v$ is directly by examining chord diagrams. The basis chord diagrams $\Gamma_{w_{i}}$ and $\left[\Gamma_{w_{i}}, \Gamma_{w_{i-1}}\right]$ are easily constructed, as in [17, Section 5]. We see $H\left[\Gamma_{w_{i}}, \Gamma_{w_{i-1}}\right]=\left[\Gamma_{w_{i+1}}, \Gamma_{w_{i}}\right]$. Hence

$$
H\left(\left[w_{i}, w_{i-1}\right]\right)=(-1)^{\left|E_{w_{i+1}}^{x}\right|}\left[w_{i+1}, w_{i}\right]
$$

for $2 \leq i \leq n-1$. We also easily compute $H w_{1}=(-1)^{\left|E_{w_{2}}^{x}\right|}\left[w_{2}, w_{1}\right], H\left[w_{n}, w_{n-1}\right]=$ $w_{n}$ and $H w_{n}=w_{1}$. It follows that

$$
H^{n+1} w_{n}=H^{n} w_{1}=\left(\prod_{i=1}^{n}(-1)^{\left|E_{w_{i}}^{x}\right|}\right) H\left[w_{n}, w_{n-1}\right]=(-1)^{\sum_{i=1}^{n}\left|E_{w_{i}}^{x}\right|} w_{n}
$$

Recall that $E_{w}^{x}$ is the set of $x$ 's in $w$ followed by $y$ 's. Now as we run through the set $w_{i}$, and pawns move from right to left, we see that for every $x$ and every $y$, they are adjacent to each other as $x y$ precisely once. Thus $\sum\left|E_{w_{i}}^{x}\right|$ counts every pair of an $x$ and a $y$ exactly once, and equals $n_{x} n_{y}$. So $H^{n+1} x^{n_{x}} y^{n_{y}}=(-1)^{n_{x} n_{y}} x^{n_{x}} y^{n_{y}}$. 
Proof of Theorem 2.6 We have shown above that for $w=w_{\min }=x^{n_{x}} y^{n_{y}}, H^{n+1} w=$ $(-1)^{n_{x} n_{y}} w$. Let now $A=\left\{w \in \mathcal{M}_{n_{x}, n_{y}}: H^{n+1} w=(-1)^{n_{x} n_{y}} w\right\}$, so $w_{\min } \in A$. That $H^{n+1}=(-1)^{n_{x} n_{y}}$ follows immediately from the following fact:

- If $w \in A$ and $w, w^{\prime}$ are related by an elementary move, then $w^{\prime} \in A$.

To see this, let $H^{n+1} w^{\prime}=\epsilon w^{\prime}$ where $\epsilon= \pm 1$. As $w, w^{\prime}$ are related by an elementary move, $w-w^{\prime}$ is a suture element, and $H^{n+1}\left(w-w^{\prime}\right)= \pm\left(w-w^{\prime}\right)$. But we have $H^{n+1}\left(w-w^{\prime}\right)=(-1)^{n_{x} n_{y}} w-\epsilon w^{\prime}=(-1)^{n_{x} n_{y}}\left(w-(-1)^{n_{x} n_{y}} \epsilon w^{\prime}\right)$. Hence we must have $(-1)^{n_{x} n_{y}} \epsilon=1$, so $\epsilon=(-1)^{n_{x} n_{y}}$ and $w^{\prime} \in A$.

Finally, it's clear from above that the least positive $j$ for which $H^{j}\left(x^{n_{x}} y^{n_{y}}\right)=$ $\pm x^{n_{x}} y^{n_{y}}$ is $j=n+1$; and $H^{n+1}=(-1)^{n_{x} n_{y}}$. Thus $H$ has period $n+1$ if $n_{x} n_{y}$ is even, and period $2 n+2$ otherwise.

\section{References}

[1] R J Baxter, Exactly solved models in statistical mechanics, Academic Press, London (1989) MR998375 Reprint of the 1982 original

[2] J Bernstein, I Frenkel, M Khovanov, A categorification of the Temperley-Lieb algebra and Schur quotients of $U\left(\mathfrak{s l}_{2}\right)$ via projective and Zuckerman functors, Selecta Math. (N.S.) 5 (1999) 199-241 MR1714141

[3] L Bombelli, J Lee, D Meyer, R D Sorkin, Space-time as a causal set, Phys. Rev. Lett. 59 (1987) 521-524 MR899046

[4] J H Conway, The sensual (quadratic) form, Carus Math. Monogr. 26, Math. Assoc. Amer., Washington, DC (1997) MR1478672 With the assistance of F Y C Fung

[5] A Frabetti, Simplicial properties of the set of planar binary trees, J. Algebraic Combin. 13 (2001) 41-65 MR1817703

[6] P Ghiggini, K Honda, J Van Horn Morris, The vanishing of the contact invariant in the presence of torsion arXiv:0706.1602

[7] E Giroux, Structures de contact en dimension trois et bifurcations des feuilletages de surfaces, Invent. Math. 141 (2000) 615-689 MR1779622

[8] E Giroux, Structures de contact sur les variétés fibrées en cercles audessus d'une surface, Comment. Math. Helv. 76 (2001) 218-262 MR1839346

[9] K Honda, On the classification of tight contact structures. II, J. Differential Geom. 55 (2000) 83-143 MR1849027

[10] K Honda, W H Kazez, G Matić, Contact structures, sutured Floer homology and TQFT arXiv:0807.2431 
[11] K Honda, W H Kazez, G Matić, Right-veering diffeomorphisms of compact surfaces with boundary, Invent. Math. 169 (2007) 427-449 MR2318562

[12] K Honda, W H Kazez, G Matić, On the contact class in Heegaard Floer homology, J. Differential Geom. 83 (2009) 289-311 MR2577470

[13] V F R Jones, Planar algebras, I arXiv:math/9909027

[14] A Juhász, Holomorphic discs and sutured manifolds, Algebr. Geom. Topol. 6 (2006) 1429-1457 MR2253454

[15] A Juhász, Floer homology and surface decompositions, Geom. Topol. 12 (2008) 299350 MR2390347

[16] P Massot, Infinitely many universally tight torsion free contact structures with vanishing Ozsváth-Szabó contact invariants arXiv:0912.5107

[17] D Mathews, Chord diagrams, contact-topological quantum field theory and contact categories, Algebr. Geom. Topol. 10 (2010) 2091-2189 MR2745667

[18] P Ozsváth, Z Szabó, Heegaard Floer homology and contact structures, Duke Math. J. 129 (2005) 39-61 MR2153455

[19] R D Sorkin, Causal sets: discrete gravity, from: "Lectures on quantum gravity", (A Gomberoff, D Marolf, editors), Ser. Cent. Estud. Cient., Springer, New York (2005) 305-327 MR2404960

[20] H N V Temperley, E H Lieb, Relations between the "percolation" and "colouring" problem and other graph-theoretical problems associated with regular planar lattices: some exact results for the "percolation" problem, Proc. Roy. Soc. London Ser. A 322 (1971) 251-280 MR0498284

Department of Mathematics, Boston College

Carney Hall, Room 301, Chestnut Hill MA 02467-3806, USA

Daniel. Mathews@bc .edu

http://www.danielmathews.info

Received: 16 February 2011 Revised: 19 June 2011 